\title{
Constraint Equations for a Planar Parallel Platform
}

by

Amani Ahmed Otaif

A thesis submitted to the Victoria University of Wellington in fulfilment of the requirements for the degree of Master of Science in Mathematics

Victoria University of Wellington

2013 



\begin{abstract}
The aim of this thesis is to apply the Grünwald-Blaschke kinematic mapping to standard types of parallel general planar three-legged platforms in order to obtain the univariate polynomials which provide the solution of the forward kinematic problem. We rely on the method of Gröbner basis to reach these univariate polynomials. The Gröbner basis is determined from the constraint equations of the three legs of the platforms. The degrees of these polynomials are examined geometrically based on Bezout's Theorem. The principle conclusion is that the univariate polynomials for the symmetric platforms under circular constraints are of degree six, which describe the maximum number of real solutions. The univariate polynomials for the symmetric platforms under linear constraints are of degree two, that describe the maximum number of real solutions.
\end{abstract}





\section{Acknowledgements}

By the name of Allah, the most Gracious and the most Compassionate

First and foremost, I would like to praise Allah the Almighty for granting me the patience, the strength and the guidance that have allowed me to accomplish this thesis.

I express my sincere appreciation to my thesis supervisor Dr. Peter Donelan who granted me this research opportunity, and whose suggestions, comments and support guided me at every step of the way. It was truly a privilege to work under his guidance.

I would like to express my deepest gratitude to my husband Mr. Mohammed Moafa, whose love, support and encouragement have been invaluable during this research. To my sons: Raeed, Abdullah and Hassan who have joined my study with their patience and whose presence in my life has given me unlimited happiness and pleasure. I owe so much thanks to my parents: my father Mr. Ahmed Otaif and my mother Mrs. Shaqra Khawaji for their prayers, extensive encouragement, endless emotional love and for providing me with an education that has inspired me to undertake this research.

Thanks must also go to Ben Clark who helped me in learning how to start using Latex, and also to Asim Masood for his help with the figures.

Last but not least, thanks are due to Saudi Arabia government for their financial support, especially their kind consideration and support towards my special needs son Raeed, which gave me the great opportunity to complete this work. 



\section{Contents}

1 Introduction 1

2 The Special Euclidean Group SE(2) 4

2.1 The Orthogonal Group $\mathrm{O}(\mathrm{n}) \ldots \ldots \ldots \ldots$

2.2 The Special Orthogonal Group $S O(n) \ldots \ldots \ldots$

2.3 Isometries . . . . . . . . . . . . . . . 8

2.4 The Euclidean Group $E(n) \ldots \ldots \ldots$

2.5 Euclidean Group as Rigid Body Transformations . . . . . . . . 15

3 Planar Kinematic Mapping $\quad 16$

3.1 Homogenous Coordinates . . . . . . . . . . . . . . 16

3.1 .1 Duality ..................... 18

3.2 The Grünwald-Blaschke Mapping . . . . . . . . . . . . 20

3.2.1 The inverse of the homogenous displacement . . . . . 25 

4 Gröbner Bases $\quad 26$

4.1 Affine Varieties and Ideals . . . . . . . . . . . . . . 27

4.2 Monomial Ordering . . . . . . . . . . . . . . 28

4.3 Gröbner bases and their Properties . . . . . . . . . . 31

4.4 Buchberger's Algorithm _. . . . . . . . . . . . . . 32

4.5 Bezout's Theorem . . . . . . . . . . . . . . . . . 33

5 Constraint Equations $\quad 36$

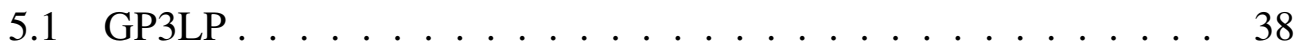

5.1.1 The Passive Sub-Chain . . . . . . . . . . . . . 40

5.2 Kinematic Constraints . . . . . . . . . . . . . . 41

5.2.1 The Lower-Pair Constraints . . . . . . . . . . . . . 42

5.2.2 The Constraint Surface Equations . . . . . . . . . . 43

5.2.3 The Formulation of GP3LP . . . . . . . . . . 49

5.3 The Forward Kinematic Problems _. . . . . . . . . . . . . 50

$5.3 .1 \quad$ C-C-C Class Legs $\ldots \ldots \ldots \ldots$. . . . . . . . 51

5.3 .2 L-L-L Class Legs $\ldots \ldots \ldots \ldots$

5.3 .3 T-T-T Class Legs $\ldots \ldots$. . . . . . . . . . . . . 60

5.4 Conclusion ......................... 62 

Appendix B

72

Appendix C

74 



\section{List of Tables}

5.1 The 18 possible leg architectures. . . . . . . . . . . . . 41

5.2 The 10 classes of GP3LP . . . . . . . . . . . . 51

5.3 Point coordinates . . . . . . . . . . . . . . 52

5.4 numerical values for point coordinates . . . . . . . . . 55

5.5 The four real platform solutions $\ldots \ldots \ldots 6$

5.6 Point coordinates . . . . . . . . . . . . . . 58

5.7 Point coordinates . . . . . . . . . . . . . . 60

5.8 The two real platform solutions . . . . . . . . . . . 61 



\section{List of Figures}

2.1 The fixed frame $\Sigma$ and the moving frame E . . . . . . . 15

5.1 A platform with three RPR legs [8] . . . . . . . . . . . . 39

5.2 The seven possible leg topologies [8]. . . . . . . . . . . 40

5.3 Hyperboloid of one sheet . . . . . . . . . . . . . . . 46

5.4 The fixed frame $\Sigma$ and the moving frame $E$ for any set of legs from Table 5.1. . . . . . . . . . . . . . . . 47

5.5 Hyperbolic Paraboloid . . . . . . . . . . . . . . 50

5.6 The four postures for RPR platform . . . . . . . . . . . 57 



\section{Chapter 1}

\section{Introduction}

Kinematic analysis of parallel robot platforms concerns both the forward kinematic problem that determines the platform poses from the positions of the actuated joints and the inverse kinematic problem which reverses these. The latter problem tends to be simpler for parallel robots. Recently, the kinematic mapping has been applied to solve the forward kinematic problem. The origin of this mapping goes back to Study whose mapping transforms the set of Euclidean displacements of space into points in a quadric in seven-dimensional projective space, called the Study quadric [4].

In 1911, Grünwald-Blaschke mapping was established independently by Grünwald and soon after by Blaschke [1]. Their writing was in German and although it was complete, it was difficult to read [10]. The Grünwald-Blaschke mapping is confined to kinematics in the plane where the set of Euclidean displacements maps to points in 3-dimensional projective space. This mapping is a special case of Study's mapping for spatial displacements [8]. The set of Euclidean displacements is considered as a point-geometry in the sense of Felix Klein's Erlangen program. His idea was: "Given any group of linear transformations in space which includes the principal (....) group as a sub-group, then the invariant theory of this group gives a definite kind of geometry, and every possible geometry can be obtained in 
this way" [8]. Hence, the Euclidean displacements are acknowledged as the basis for Euclidean geometry.

In 1979, the Grünwald-Blaschke mapping was reintroduced by Bottema and Roth [1]. It motivated researchers to examine manipulators kinematically via such a mapping. Ravani and Roth [15] in 1983 approximated the motion of a planar manipulator using the kinematic mapping. Husty [18] in 1994 used this approach of Bottema and Roth to find univariate polynomials of the forward kinematic problem for planar and spherical manipulators. Hayes and Chen attempted to find the solution for the forward kinematic problem for all planar manipulators in 1999 and $2001[2,9]$.

The Kinematic mapping is algebraic since it involves polynomials. Then, methods of algebraic geometry such as Gröbner bases provide an accessible method to extract the univariate polynomials for the forward kinematic problem [14]. Moreover, Bezout's Theorem is an unequivocal way to enumerate the solutions of the forward kinematic problem.

In this research, we focus on describing in detail the solution of the forward kinematic problem for symmetric fully-parallel planar three-legged platforms. Each of the three legs constrains the motion of the platform. This type of constrained motion is determined by the types of joints in the legs. Since we are here interested in a planar platform, the joints are restricted to be of revolute and prismatic types. Then the constraint motion can be shown to be either linear or circular. The solution of the forward kinematic problem is based on the Grünwald-Blaschke mapping. Hence, to introduce an explanation of this mapping is one of the goals of this thesis. Then, after we have applied the Grünwald-Blaschke mapping to a platform, the constraints imposed by the three legs are transformed into constraint surfaces in the image space. The set of Euclidean displacements $S E(2)$ is the domain of this mapping. It is a three-dimensional Lie group for which the mapping provides an algebraic description. 
Gröbner bases are our method to solve the forward kinematic problem. The Gröbner basis is obtained from the set of the three constraint surfaces, each defined by an algebraic equation, namely the images of the constrained displacements via the kinematic mapping.

The general planar parallel three-legged platforms are of 10 classes [2]. They are divided into symmetric and asymmetric platforms. The symmetric parallel platforms are of three classes and they are the specific objects of our work. One of these is regarded as a circular constraint while the other two are the inversion of each other and they are corresponded to a linear constraint. The solution for the forward kinematic problem in this thesis is attempted for these symmetric classes. For a platform with circular constraint, the univariate polynomial is of order six whereas the univariate polynomial for a platform with linear constraint is of order two. This agrees with what has been found by Merlet [13]. In terms of algebraic geometry, we employ Bezout's Theorem to clarify how this solution relates to the geometry [9].

The structure of this thesis is as follows. In Chapter 2, we present the set of Euclidean displacements $S E(n)$ and in particular $S E(2)$. We also explain the map that describe the Euclidean displacements through two frames of coordinate systems. Chapter 3 gives details of Grünwald-Blaschke mapping. A brief introduction to the method of Gröbner bases is in Chapter 4. We include an algorithm for constructing it and also state Bezout's Theorem. In Chapter 5 we introduce the general planar three-legged platforms. The Grünwald-Blaschke mapping and algebraic geometry tools are utilized to solve the forward kinematic problems, specifically by implementing them on the symmetric parallel planar manipulators. Then, we determine the circular constraint equation and the linear constraint equation for such manipulators. These equations are the point equations for the constraint surfaces in the image space. The forward kinematic problem is then solved for the three symmetric platforms and we conclude by a discussion of the solution via Bezout's Theorem. 


\section{Chapter 2}

\section{The Special Euclidean Group SE(2)}

This chapter represents an introduction to the group $S E(n)$, in particular $S E(2)$, where $S E(n)$ is the set of Euclidean displacements of n-dimensional Euclidean space. The group $S E(n)$ is a sub-group of the group of transformations whose members are isometries. To begin with, fundamental concepts from linear algebra are reviewed as follows:

Definition 2.0.1 Let $V_{1}$ and $V_{2}$ be real vector spaces. A map

$$
T: V_{1} \longrightarrow V_{2}
$$

is called a linear transformation if it satisfies the following properties:

1. $\forall u, w \in V_{1}, T(u+w)=T(u)+T(w)$.

2. $\forall u \in V_{1}, a \in \mathbb{R}, T(a u)=a T(u)$.

Let the set of all $m \times n$ real matrices be denoted by $M(m, n)$. The set $M(m, n)$ with two operations matrix addition and scalar multiplication is a vector space. A linear transformation can be represented by a matrix form which can be seen by the following theorem: 
Theorem 2.0.2 A linear transformation $T: V_{1} \longrightarrow V_{2}$ can be represented by a matrix $A \in M(m, n)$ where $\operatorname{dim} V_{1}=n$ and $\operatorname{dim} V_{2}=m$ as

$$
T\left(e_{j}\right)=\sum_{i=1}^{m} a_{i j} f_{i} \quad j=1, \ldots, n
$$

where the sets $e_{1}, \ldots, e_{n}$, and $f_{1}, \ldots, f_{m}$ are bases for the vector spaces $V_{1}, V_{2}$ respectively and the $a_{i j}$ form an $m \times n$ matrix $A$.

\subsection{The Orthogonal Group O(n)}

In this section the concept of an orthogonal transformation will be discussed and we will show how the orthogonal group can be decomposed into two classes which make the orthogonal group $O(n)$ a disconnected Lie group. The Lie group was introduced by the mathematician Sophus Lie $(1842-1899)$ and it is defined to be a smooth manifold $G$ which is also a group with the group operations:

$$
\begin{gathered}
\cdot G \times G \longrightarrow G \\
{ }^{-1}: G \longrightarrow G
\end{gathered}
$$

being continuous differentiable mappings. (For more information on Lie groups see [16]). First, recall that the inner product in $\mathbb{R}^{n}$ is defined as follows:

$$
\langle\boldsymbol{x}, \boldsymbol{y}\rangle=\boldsymbol{x}^{T} \boldsymbol{y}=\left(x_{1} y_{1}+x_{2} y_{2}+\ldots+x_{n} y_{n}\right) .
$$

Definition 2.1.1 The orthogonal group $O(n)$ is defined to be the set of linear transformations of the vector space $\mathbb{R}^{n}$ that preserve the inner product and is written:

$$
O(n)=\left\{A \in M(n, n) \mid \forall x, y \in \mathbb{R}^{n},\langle A x, A y\rangle=\langle x, y\rangle\right\}
$$

Since the elements of this group are represented by $n \times n$ matrices, then, we can use the following theorem to show that $O(n)$ is a group. From the point of view of the group definition, $I_{n}$ the $n \times n$ identity matrix, and matrix multiplication is the group operation. 
Theorem 2.1.2 $A \in O(n)$ if and only if $A^{t} A=I_{n}$.

Proof. $\Rightarrow$ Let $A \in O(n)$, then A preserves the inner product by definition. Let the set $\left\{e_{1}, \ldots, e_{n}\right\}$ be the standard basis for the vector space $\mathrm{V}$. The vector $e_{i}$ has 1 in the ith entry and zero elsewhere, so we have:

$$
\left\langle e_{i}, e_{j}\right\rangle=\delta_{i j}= \begin{cases}1 & \text { if } i=j \\ 0 & \text { otherwise }\end{cases}
$$

As $A$ preserves the inner product, this gives:

$$
\left\langle A e_{i}, A e_{j}\right\rangle=\left\langle e_{i}, e_{j}\right\rangle=\delta_{i j}= \begin{cases}1 & \text { if } i=j \\ 0 & \text { otherwise }\end{cases}
$$

Now $A e_{i}$ clearly expresses the $i$ th column of $A$ which is equivalent to the $i$ th row of $A^{t}$. Hence, for all $i, j=1, \ldots, n$ :

$$
\left\langle A e_{i}, A e_{j}\right\rangle=\left(A e_{i}\right)^{t}\left(A e_{j}\right)=e_{i}^{t} A^{t} A e_{j}=\left(A^{t} A\right)_{i j}=I_{i j}
$$

which implies $A^{t} A=I$.

$\Leftarrow$ If $A^{t} A=I$, then for any $i j$ th element we have $\left(A^{t} A\right)_{i j}=I_{i j}$. Since $e_{i}^{t} e_{j}=I_{i j}$ for the standard basis $e_{1}, \ldots, e_{n}$, then $\left(A e_{i}\right)^{t}\left(A e_{j}\right)=e_{i}^{t} e_{j}$ which shows that $A$ preserves the inner product and hence it is an orthogonal matrix.

To show that $O(n)$ is in fact a group we need to check that it satisfies the group axioms: Let $A, B \in O(n)$. Since the matrix multiplication is associative, we only need to check that $A^{-1}$ and $A B \in O(n)$. As $A \in O(n)$ implies $A^{t} A=I$, then, $A^{t}=A^{-1}$. Now,

$$
\left(A^{-1}\right)^{t} A^{-1}=\left(A^{t}\right)^{t} A^{t}=\left(A^{t} A\right)^{t}=I^{t}=I
$$

Thus, $A^{-1} \in O(n)$. Next, since $A, B \in O(n)$, then $A^{t} A=I$ and $B^{t} B=I$. Now,

$$
(A B)^{t}(A B)=\left(B^{t} A^{t}\right)(A B)=B^{t}\left(A^{t} A\right) B=B^{t}(I) B=B^{t} B=I .
$$

Hence, $O(n)$ is a group. An element $A$ of $O(n)$ satisfies $A^{t} A=I$. Applying the determinant to this equation and using the following properties of the determinant: 
(i) $\operatorname{det}(A B)=\operatorname{det}(A) \operatorname{det}(B)$

(ii) $\operatorname{det}(A)=\operatorname{det}\left(A^{T}\right)$

leads to a crucial result which is $\operatorname{det}(A)^{2}=1$, which implies $\operatorname{det}(A)= \pm 1$.

As a consequence, the orthogonal group $O(n)$ consists of two disconnected classes, those matrices that have $\operatorname{det}(A)=+1$ and those that have $\operatorname{det}(A)=-1$. They are rotations about the origin in $n$-dimensions and reflections in a hyperplane that passes through the origin, respectively. The rotation component involves the identity element and thus it is a subgroup of $O(n)$, denoted $S O(n)$, and it is of particular interest in robotics, so it is treated specifically in the following section.

\subsection{The Special Orthogonal Group $S O(n)$}

The special orthogonal group is the following subgroup of $O(n)$ :

$$
S O(n)=\{A \in M(n, \mathbb{R}) \mid A \in O(n), \operatorname{det} A=1\}
$$

We begin our analysis by considering the components in two dimensions as they concern planar motions and this is what we are interested in.

When $n=2, O(2)$ consists of matrices of the form

$$
\left[\begin{array}{cc}
\cos \phi & -\sin \phi \\
\sin \phi & \cos \phi
\end{array}\right],\left[\begin{array}{cc}
\cos \phi & \sin \phi \\
\sin \phi & -\cos \phi
\end{array}\right], \quad \text { where } \phi \in \mathbb{R}
$$

The first type of matrix constitutes the subgroup formed by the anti-clockwise rotations by $\phi$ about the origin whereas the second type constitutes the reflections in lines making angle $\phi$ with the $\mathrm{x}$-axis. Our interest is in the first type.

To clarify how these matrices are formed we can derive them: 
Consider $A \in O(2)$ such that

$$
A=\left[\begin{array}{ll}
a & b \\
c & d
\end{array}\right] .
$$

Since $A \in O(2)$, then $A^{t} A=I$ which implies that $a^{2}+c^{2}=1, b^{2}+d^{2}=1$, and $a b+c d=0$. This gives $|a| \leq 1$ and so $a=\cos \phi$ for some $\phi \in \mathbb{R}$ and then, $c^{2}=1-\cos \phi^{2}=\sin ^{2} \phi \Rightarrow c= \pm \sin \phi$. By using the trigonometric identities and if necessary replacing $\phi$ by $2 \pi-\phi$ we can always have $c=+\sin \phi$. This is done without loss of generality since $\cos ^{2} \phi+\sin ^{2} \phi=1$. Moreover, $a b+c d=0$ implies $b \cos \phi+d \sin \phi=0$. Now, in the case where $\operatorname{det} A=1$ we have $a d-b c=1$. Solving the last two equations simultaneously gives: $d=\cos \phi$ and $b=-\sin \phi$ while in the case $\operatorname{det} A=-1$ we have $a d-b c=-1$ which yields $d=-\cos \phi$ and $b=\sin \phi$. Then, we can choose $\lambda \neq 0$ such that $b=-\lambda \sin \phi, d=\lambda \cos \phi$ where $\lambda=1$ in the case $\operatorname{det} A=1$. This gives the matrices form as presented above.

\subsection{Isometries}

The word isometry comes from the Greek isometros, meaning 'equal measure'.

Definition 2.3.1 An isometry(or rigid transformation) $T: \mathbb{R}^{n} \longrightarrow \mathbb{R}^{n}$ of $n$-dimensional space $\mathbb{R}^{n}$ is a transformation that preserves the Euclidean metric (distance). That is, for all $u, v \in \mathbb{R}^{n}$,

$$
\|T(u)-T(v)\|=\|u-v\|
$$

Isometries can be obtained in several ways:

(i) Translation by adding a constant vector say $d$ to the corresponding vectors:

$$
T_{d}: u \longrightarrow u+d
$$

(ii) Rotation by multiplying the vectors by an orthogonal matrix $A$ :

$$
R_{A}: u \longrightarrow A u, \quad \operatorname{det} A=1
$$


(iii) Reflection by multiplying the vectors by orthogonal matrix $A$ that has its form:

$$
F_{A}: u \longrightarrow A u, \quad \operatorname{det} A=-1
$$

These are examples of isometries on the plane and in fact in any dimension. Furthermore, the composition of two isometries is also an isometry. This follows from the definition, that is if $T$ and $H$ are isometries, then

$$
\begin{aligned}
& \|(T \circ H)(u)-((T \circ H)(v) \| \\
& =\|T(H(u))-T(H(v))\| \\
& =\|H(u)-H(v)\| \quad \text { as } T \text { is an isometry } \\
& =\|u-v\| \quad \text { as } \quad H \quad \text { is an isometry. }
\end{aligned}
$$

In order to perform a combination of a translation and a rotation suppose $u$ is any vector in the plane. Then, multiply it by an orthogonal matrix A, say. We get

$$
u^{\prime}=A u
$$

Next, we add a constant vector $d \in \mathbb{R}^{n}$ to the resulting vector. We have

$$
u^{\prime \prime}=u^{\prime}+d=A u+d
$$

This equation shows the transformations that can be written as pairs $(A, d)$. The following proposition will show these pairs are the only isometries.

Proposition 2.3.2 Any isometry $T$ of $\mathbb{R}^{n}$ is the composition of a translation with an orthogonal transformation [5].

Proof. Let $T$ denote an isometry in $\mathbb{R}^{n}$. We assume $T(0)=u$. By looking at the composition of $T_{-u}$ with $T$, this composition will leave 0 fixed. Let $H$ denote this composition and we claim any isometry $H$ that leaves 0 fixed is orthogonal. To prove this we need to show $H$ respects the following: 
(i) norms: since $H$ is an isometry, then,

$$
\begin{aligned}
\|u\|=\|u-0\| & =\|H(u)-H(0)\| \\
& =\|H(u)\| \text { for some } u \in \mathbb{R}^{n} .
\end{aligned}
$$

(ii) inner product: for any $u, v \in \mathbb{R}^{n}$

$$
\begin{aligned}
\|H(u)-H(v)\|^{2} & =\|u-v\|^{2} \quad \text { as } H \text { is an isometry } \\
L H S & =\langle H(u)-H(v), H(u)-H(v)\rangle \quad \text { by definition of norm } \\
& =\langle H(u), H(v)\rangle-2\langle H(u), H(v)\rangle+\langle H(v), H(v)\rangle
\end{aligned}
$$

(as inner product is bilinear and symmetric)

$$
\begin{aligned}
& =\|H(u)\|^{2}-2\langle H(u), H(v)\rangle+\|H(v)\|^{2} \\
& =\|u\|^{2}-2\langle H(u), H(v)\rangle+\|v\|^{2}
\end{aligned}
$$

Similarly,

$$
R H S=\|u\|^{2}-2\langle u, v\rangle+\|v\|^{2}
$$

Hence,

$$
\langle H(u), H(v)\rangle=\langle u, v\rangle
$$

(iii) $H$ is linear: To prove this, the two conditions for linearity $H(\lambda u)=\lambda H(u)$ and $H(u+v)=H(u)+H(v)$ must hold where $u, v \in \mathbb{R}^{n}$ and $\lambda \in \mathbb{R}$. To verify this:

$$
\begin{aligned}
\|H(\lambda u)-\lambda H(u)\|^{2} & =\langle H(\lambda u)-\lambda H(u), H(\lambda u)-\lambda H(u)\rangle \\
& =\langle H(\lambda u), H(\lambda u)\rangle-2\langle H(\lambda u), \lambda H(u)\rangle+\langle\lambda H(u), \lambda H(u)\rangle \\
& =\|H(\lambda u)\|^{2}-2\langle H(\lambda u), \lambda H(u)\rangle+\|\lambda H(u)\|^{2} \\
& =\|H(\lambda u)\|^{2}-2 \lambda\langle H(\lambda u), H(u)\rangle+\lambda^{2}\|H(u)\|^{2} \\
& =\|\lambda u\|^{2}-2 \lambda\langle\lambda u, u\rangle+\lambda^{2}\|u\|^{2} \text { by (ii) } \\
& =\lambda^{2}\|u\|^{2}-2 \lambda^{2}\langle u, u\rangle+\lambda^{2}\|u\|^{2}=0
\end{aligned}
$$


This is done by using the bilinearity of inner product. Therefore, $H(\lambda u)=$ $\lambda H(u)$. To verify the other condition of linearity:

$$
\begin{aligned}
\| H(u+v)- & H(u)-H(v) \|^{2} \\
= & \langle H(u+v)-H(u)-H(v), H(u+v)-H(u)-H(v)\rangle \\
= & \langle H(u+v), H(u+v)\rangle-\langle H(u+v), H(u)\rangle-\langle H(u+v), H(v)\rangle \\
& -\langle H(u), H(u+v)\rangle+\langle H(u), H(v)\rangle+\langle H(u), H(v)\rangle \\
& -\langle H(v), H(u+v)\rangle+\langle H(u), H(v)\rangle+\langle H(v), H(v)\rangle
\end{aligned}
$$

As $H$ is an isometry, by (ii) that this equals:

$$
\begin{aligned}
& \|u+v\|^{2}-2\langle u+v, u\rangle-2\langle u+v, v\rangle+\|u\|^{2}+2\langle u, v\rangle+\|v\|^{2} \\
& =\|u+v\|^{2}-2(\langle v, u\rangle+\langle v, u\rangle)-2(\langle u, v\rangle+\langle v, v\rangle) \\
& \quad+\|a\|^{2}+2\langle u, v\rangle+\|v\|^{2} \\
& =\|u+v\|^{2}-2\|a\|^{2}-2\langle v, u\rangle-2\langle v, u\rangle-2\|v\|^{2} \\
& \quad \quad+\|u\|^{2}+2\langle u, v\rangle+\|v\|^{2} \\
& =\|u+v\|^{2}-\|u\|^{2}-\|v\|^{2}-2\langle v, u\rangle \\
& =\|u+v\|^{2}-\left(\|u\|^{2}+\|v\|^{2}+2\langle v, u\rangle\right) \\
& =\|u+v\|^{2}-\|u+v\|^{2} \quad \text { since } \quad\|u+v\|^{2}=\|u\|^{2}+\|v\|^{2}+2\langle v, u\rangle \\
& =0
\end{aligned}
$$

thus, $H(u+v)=H(u)+H(v)$.

The two conditions of linearity are satisfied because $u=0 \Leftrightarrow\|u\|=0$ Hence, this completes the proof that $H$ is orthogonal.

\subsection{The Euclidean Group $E(n)$}

The Euclidean group $E(n)$ is defined to be the group of all isometries of the Euclidean space $\mathbb{R}^{n}$. This includes translations, rotations and reflections. An 
isometry $T$ is a bijective map. That is, it is injective since if $u, v \in \mathbb{R}^{n}$ and assume $T(u)=T(v)$, then

$$
\begin{aligned}
T(u)-T(v)=0 & \Rightarrow\|T(u)-T(v)\| \\
& =\|0\|=0 \\
& \Rightarrow\|u-v\|=0 \quad \text { as } T \text { is an isometry } \\
& \Rightarrow u-v=0 \\
& \Rightarrow u=v
\end{aligned}
$$

and it is surjective (a proof of this can be found using proposition 2.3.2, for example). This asserts an inverse $T^{-1}$ of an isometry $T$ exists. It is also an isometry. For given $u, v \in \mathbb{R}^{n}$, as $T$ is surjective, then there exist $w, q$ such that $T(w)=u$ and $T(q)=v$. Then,

$$
\begin{aligned}
\left\|T^{-1}(u)-T^{-1}(v)\right\| & =\left\|T^{-1}(T(w))-T^{-1}(T(q))\right\| \\
& =\|w-q\|=\|T(w)-T(q)\| \quad \text { as } \quad T \quad \text { is an isometry } \\
& =\|u-v\| .
\end{aligned}
$$

This shows the closure in $E(n)$ under inverse is satisfied. In addition, since the translation by the vector $\mathbf{0}$ in $E(n)$ leaves each element in its place, then, $E(n)$ has an identity element denoted by $T_{0}$ where the composition of any isometry $T$ with $T_{0}$ gives the isometry $T$ again. That is:

$$
T \circ T_{0}=T_{0} \circ T=T .
$$

Furthermore, we have just stated above by $\operatorname{Eq}(2.1)$ that $E(n)$ is closed under composition and the composition operation always satisfies the associativity. All these properties proves $E(n)$ is a group. To understand the structure of this group, we recall the following definition:

Definition 2.4.1 Given two groups $G$ and $K$, their direct product is defined to be the Cartesian product set $G \times K$ of pairs $(g, k)$ with group operation for all $\left(g_{i}, k_{i}\right) \in G \times K, i=1,2$,

$$
\left(g_{1}, k_{1}\right) \cdot\left(g_{2}, k_{2}\right)=\left(g_{1} k_{1}, g_{2} k_{2}\right) .
$$


If $G$ is a group and $V$ a vector space on which $G$ acts, then the semi-direct product $G \ltimes V$ is the group combining the operation on $G$, addition on $V$ and the group action, as follows. For all $\left(g_{i}, v_{i}\right) \in G \ltimes V, i=1,2$

$$
\left(g_{1}, v_{1}\right) \cdot\left(g_{2}, v_{2}\right)=\left(g_{1} g_{2}, g_{1} v_{2}+v_{1}\right)
$$

The Euclidean group is a semi-direct product with $G=O(n)$ and $V=\mathbb{R}^{n}$. To verify the operation of the Euclidean group $E(n)$ : If we look at the sequence of two successive transformations of $E(n)$ on a single vector $u \in \mathbb{R}^{n}$ :

$$
\begin{aligned}
& \left(A_{1}, d_{1}\right)(u)=A_{1} u+d_{1} \quad \text { and then } \\
& \left(A_{2}, d_{2}\right)\left(A_{1} u+d_{1}\right) \\
& =A_{2} A_{1} u+A_{2} d_{1}+d_{2}
\end{aligned}
$$

Hence, the product of two of these transformations is:

$$
\left(A_{2}, d_{2}\right)\left(A_{1}, d_{1}\right)=\left(A_{2} A_{1}, A_{2} d_{1}+d_{2}\right)
$$

where $A_{1}, A_{2} \in O(n), d_{1}, d_{2} \in \mathbb{R}^{n}$. Group elements of the form $\left(I_{n}, d\right)$ are translations in $\mathbb{R}^{n}$. In other words, each vector in the plane or space is translated by the vector $d$. Elements of the form $(A, 0)$ are rotations about the origin or reflections in a hyperplane through the origin. In fact every element except the translation is a rotation about some point. This group of isometries can be represented in a block matrix form as follows:

$$
E(n)=\left\{\left[\begin{array}{cc}
A & d \\
0 & 1
\end{array}\right] \in G L(n+1, \mathbb{R}) \mid d \in \mathbb{R}^{n}, A \in O(n)\right\}
$$

where $G L(n+1, \mathbb{R})$ is the general linear lie group of degree $n$ over $\mathbb{R}$ and its elements are matrices with non zero determinant and $O(n)$ is the group of orthogonal matrices. This operation can be expressed by $(n+1) \times(n+1)$ matrix multiplication as:

$$
\left[\begin{array}{cc}
A_{2} & d_{2} \\
0 & 1
\end{array}\right]\left[\begin{array}{cc}
A_{1} & d_{1} \\
0 & 1
\end{array}\right]=\left[\begin{array}{cc}
A_{2} A_{1} & A_{2} d_{1}+d_{2} \\
0 & 1
\end{array}\right]
$$


This is called the homogenous represenation of $E(n)$.

We already know that the group $O(n)$ has two disjoint classes. Orthogonal matrices with determinant 1 correspond to rotations about the origin in $\mathbb{R}^{n}$. They form the group $S O(n)$. Orthogonal matrices with determinant -1 correspond to reflections. In robotics, there is no interest in the transformations that reverse the orientation of a basis of vectors, so our concerns are confined to the subgroup of proper rigid body transformations, namely, the special Euclidean group of isometries $S E(n)=S O(n) \ltimes \mathbb{R}^{n}[16]$ which is in set form written:

$$
S E(n)=\left\{(A, d): A \in S O(n), d \in \mathbb{R}^{n}\right\}
$$

In particular we are interested in the case $n=2$ since our application is to planar robots. The elements of the group $S E(2)$ consist of a $2 \times 2$ rotation matrix represented by an angle $\phi$, and a translation vector in the plane $\mathbb{R}^{2}$. Therefore, $S E(2)$ is a 3-dimensional Lie group. In other words, $S E(2)$ is represented by a 3-dimensional space. Moreover, $S E(2)$ is called the group of proper rigid body motions in the plane which can be generated by a mechanism with three degrees of freedom, where the degree of freedom of a manipulator is defined as the number of its independent movements and it is shortened to DOF.

In the homogenous representation, the elements of $S E(2)$ geometrically act on the plane $z=1$ in $\mathbb{R}^{3}$. They have the matrix form

$H=\left[\begin{array}{ccc}\cos \phi & -\sin \phi & a \\ \sin \phi & \cos \phi & b \\ 0 & 0 & 1\end{array}\right], \quad$ where $\quad A=\left[\begin{array}{cc}\cos \phi & -\sin \phi \\ \sin \phi & \cos \phi\end{array}\right] \in S O(2), d=\left[\begin{array}{l}a \\ b\end{array}\right] \in \mathbb{R}^{2}$

which can be expressed in terms of linear matrix representation:

$$
H \cdot\left[\begin{array}{l}
x \\
y \\
1
\end{array}\right]=\left[A\left[\begin{array}{l}
x \\
y
\end{array}\right]+d\right]
$$




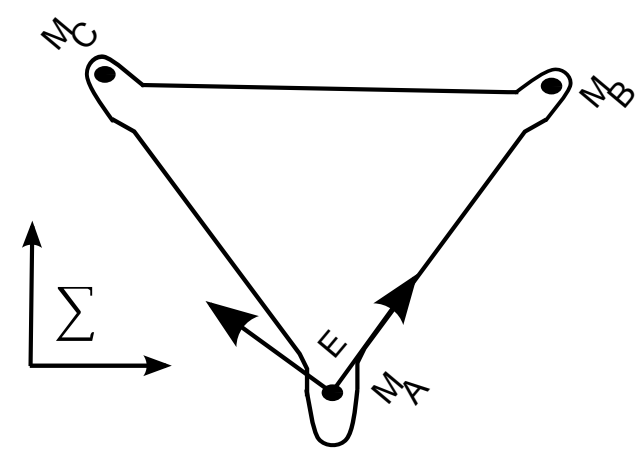

Figure 2.1: The fixed frame $\Sigma$ and the moving frame $\mathrm{E}$

\subsection{Euclidean Group as Rigid Body Transformations}

Consider a rigid body in $\mathbb{R}^{n}(n=2$ or 3$)$. Then, there are two frames of coordinate systems to acknowledge for a moving rigid body: moving coordinate frame and fixed, or base, coordinate frame. In kinematics, the moving frame is the space attached to the body itself and a fixed coordinate system is the physical space of a mechanism itself namely, the space where it is located [10]. If $E$ denotes the moving coordinate system and $\Sigma$ denotes the fixed coordinate system, then they are linked by the map

$$
\gamma: X \longrightarrow A x+d
$$

where $\gamma: \mathbb{R}^{n} \longrightarrow \mathbb{R}^{n}$; see Fig. 2.1. This is the Euclidean displacement mapping that describes a displacement of the moving coordinate system $E$ relative to the fixed system $\Sigma$, and it is standard for representing a n-dimensional displacement where $A$ is a proper orthogonal rotation matrix, $d$ is a translation vector and $X$, $x$ are coordinates for a point in $\Sigma$ and $E$ respectively. The coordinate $X$ is thus a coordinate of $x$ by such a transformation. Therefore, an element of $E(n)$ is described by such a map since $E(n)$ is the set of Euclidean displacements. 


\section{Chapter 3}

\section{Planar Kinematic Mapping}

Essentially, kinematics is the study of Euclidean displacement or any motion of a body in a space. In this chapter the idea of mapping the set of proper planar isometries onto the points of 3 -dimensional projective space $P^{3}(\mathbb{R})$ is presented. This was derived independently in 1911 by Grünwald and soon after by Blaschke [1]. Hence, in order to introduce the Grünwald-Blaschke mapping, we recall the motion for a rigid body in a plane as mentioned in chapter 2 . Three independent parameters are demanded to describe this motion since its displacements can occur by translating the rigid body in two directions $x$-axis and $y$-axis or by rotating it in one direction about a point in the plane. Thus, the number of degrees of freedom DOF for a rigid body in a plane is three. As a preliminary we start with some of the fundamental concepts of the 3-dimensional projective space $P^{3}(\mathbb{R})$ and its homogenous coordinates. We follow the description of projective space in Cox et al [3], Galarza et al [5] and Chen [2].

\subsection{Homogenous Coordinates}

This section will introduce the notion of projective space and its homogenous coordinates that are useful for the kinematic mapping. The intersection of two lines 
in the plane $\mathbb{R}^{2}$ occurs in one point but this does not include parallel lines which do not meet in $\mathbb{R}^{2}$. In the projective plane these parallel lines will meet at points at infinity which are determined by the direction of these lines. This gives a guide to defining the projective space $P^{n}(\mathbb{R})$ as follows.

In $\mathbb{R}^{n+1}$, the relation $\sim$ on two nonzero points $\left(x_{1}, x_{2}, x_{3}, \ldots, x_{n}, x_{n+1}\right)$, $\left(y_{1}, y_{2}, y_{3}, \ldots, y_{n}, y_{n+1}\right)$ is defined by:

$$
\begin{gathered}
\left(x_{1}, x_{2}, x_{3}, \ldots, x_{n}, x_{n+1}\right) \sim\left(y_{1}, y_{2}, y_{3}, \ldots, y_{n}, y_{n+1}\right) \quad \text { if and only if } \\
\left(x_{1}, x_{2}, x_{3}, \ldots, x_{n}, x_{n+1}\right)=\lambda\left(y_{1}, y_{2}, y_{3}, \ldots, y_{n}, y_{n+1}\right)
\end{gathered}
$$

where $\lambda$ is a nonzero real number. It is easy to show that the relation $\sim$ is reflexive, symmetric and transitive. Therefore, $\sim$ is an equivalence relation on $\mathbb{R}^{n+1}-\{\boldsymbol{0}\}$ and we say the two nonzero points $\left(x_{1}, x_{2}, x_{3}, \ldots, x_{n}, x_{n+1}\right),\left(y_{1}, y_{2}, y_{3}, \ldots, y_{n}, y_{n+1}\right)$ are equivalent. The equivalence class of $\left(x_{1}, x_{2}, x_{3}, \ldots, x_{n}, x_{n+1}\right)$ is therefore the set

$$
\left[\left(x_{1}, x_{2}, x_{3}, \ldots, x_{n}, x_{n+1}\right)\right]=\left\{\lambda\left(x_{1}, x_{2}, x_{3}, \ldots, x_{n}, x_{n+1}\right) \mid \lambda \in \mathbb{R}, \lambda \neq 0\right\} .
$$

Definition 3.1.1 The $\mathbf{n}$-dimensional projective space over the field $\mathbb{R}$, denoted $P^{n}(\mathbb{R})$, is the set of equivalence classes of $\sim$ on $\mathbb{R}^{n+1}-\{\boldsymbol{0}\}$ and we write:

$$
P^{n}(\mathbb{R})=\left(\mathbb{R}^{n+1}-\{\mathbf{0}\}\right) / \sim
$$

The coordinates $\left(x_{1}, x_{2}, x_{3}, \ldots, x_{n+1}\right) \in \mathbb{R}^{n+1}$ of a point $p \in P^{n}(\mathbb{R})$ are its homogenous coordinates and are written as ratios $\left(x_{1}: x_{2}: x_{3}: \ldots: x_{n+1}\right)$ to distinguish them from the coordinates of a point in $\mathbb{R}^{n+1}$. The homogenous coordinates of a point in $P^{n}(\mathbb{R})$ are thus not unique, since any non-zero multiple represents the same point $p$.

Geometrically, the points of $P^{n}(\mathbb{R})$ correspond to lines through the origin in $\mathbb{R}^{n+1}$ (excluding $\mathbf{0}$ ). To help our thinking of this notion let us for instance consider $P^{2}(\mathbb{R})$. Every point in the plane $x_{3}=1$ will meet a unique line through the origin 
in $\mathbb{R}^{3}$. Thus, these lines represent a point in $P^{2}(R)$ with homogenous coordinates $(x: y: 1)$.

There is a relationship between the cartesian coordinates $(x, y)$ in this plane and its homogenous coordinates $\left(x_{1}: x_{2}: x_{3}\right)$, given by:

$$
x=\frac{x_{1}}{x_{3}}, y=\frac{x_{2}}{x_{3}} .
$$

In the case $x_{3}=0$, the point $\left(x_{1}: x_{2}: 0\right)$ corresponds to a point at infinity. Such points form a projective line by treating $\left(x_{1}: x_{2}\right)$ as homogenous coordinates for $P^{1}(\mathbb{R})$. In 3-dimensional projective space, a similar correspondence applies with a relationship between cartesian coordinates $(x, y, z)$ and homogenous coordinates $\left(x_{1}: x_{2}: x_{3}: x_{4}\right)$ given by:

$$
x=\frac{x_{1}}{x_{4}}, y=\frac{x_{2}}{x_{4}}, z=\frac{x_{3}}{x_{4}}
$$

where the case $x_{4}=0$ represents a point on a plane at infinity.

\subsubsection{Duality}

One of the fundamental principles in mathematics is duality. It plays a role in many areas, such as geometry. Fundamentally, the equation of a line in $\mathbb{R}^{2}$ is:

$$
L_{1} x+L_{2} y+L_{3}=0, \quad L_{1} \quad \text { or } \quad L_{2} \neq 0
$$

where $(x, y)$ are the cartesian coordinates for any point on this line and $L_{1}, L_{2}$ and $L_{3}$ are arbitrary constants that determine the slope and the intersections with the coordinate axes. Rewriting this equation in terms of the homogenous coordinates $\left(x_{1}: x_{2}: x_{3}\right)$ of $(x, y)$ we have:

$$
\begin{array}{r}
\frac{x_{1}}{x_{3}} L_{1}+\frac{x_{2}}{x_{3}} L_{2}+L_{3}=0 \Leftrightarrow \\
x_{1} L_{1}+x_{2} L_{2}+x_{3} L_{3}=0
\end{array}
$$


Eq.(3.1) is the homogenous line equation defining a line in $P^{2}(\mathbb{R})$. Treating the parameters $\left(L_{1}, L_{2}, L_{3}\right)$ and the homogenous coordinates as vectors in $\mathbb{R}^{3}$, the dot product of $\left(L_{1}, L_{2}, L_{3}\right)$ and any point on the line vanishes:

$$
\left(L_{1}, L_{2}, L_{3}\right) \cdot\left(x_{1}, x_{2}, x_{3}\right)=0
$$

Clearly, this line can be specified by any non-zero multiple of $\left(L_{1}, L_{2}, L_{3}\right)$. Then, $\left(L_{1}: L_{2}: L_{3}\right)$ can be regarded as homogenous coordinates for the projective line, corresponding geometrically to a plane through the origin in $\mathbb{R}^{3}$. Then, any point $\left(x_{1}: x_{2}: x_{3}\right)$ that fulfills Eq. (3.2) is incident with the line $\left(L_{1}: L_{2}: L_{3}\right)$ and any line $\left(L_{1}: L_{2}: L_{3}\right)$ that fulfills Eq. (3.2) is incident with the point $\left(x_{1}: x_{2}: x_{3}\right)$. Hence, points and lines are dual objects in $P^{2}(\mathbb{R})$. Similarly, an equation for a plane in 3-dimensional space $\mathbb{R}^{3}$ is given by

$$
L_{1} x+L_{2} y+L_{3} z+L_{4}=0
$$

where $(x, y, z)$ are the cartesian coordinates for a point in $\mathbb{R}^{3}$ on this line. The corresponding projective equation is given by:

$$
L_{1} x_{1}+L_{2} x_{2}+L_{3} x_{3}+L_{4} x_{4}=0
$$

where $\left(x_{1}: x_{2}: x_{3}: x_{4}\right)$ are homogenous coordinate of a point in $P^{3}(\mathbb{R})$. Using the dot product in $\mathbb{R}^{4}$ the projective line equation is written:

$$
\left(L_{1}, L_{2}, L_{3}, L_{4}\right) \cdot\left(x_{1}, x_{2}, x_{3}, x_{4}\right)=0
$$

and the equivalence class of $\left(L_{1}, L_{2}, L_{3}, L_{4}\right)$ now determines a plane, hence they provide homogenous coordinates of this plane. Then, any point that satisfies Eq. (3.3) is incident with the plane $\left(L_{1}: L_{2}: L_{3}: L_{4}\right)$ and any plane $\left(L_{1}: L_{2}: L_{3}: L_{4}\right)$ that satisfies Eq. (3.3) is incident with the point $\left(x_{1}: x_{2}: x_{3}: x_{4}\right)$. Therefore, points and planes are dual objects in projective 3 -space [5].

In addition, another well known way that Eq.(3.1) can be rewritten is by using the determinant. Then, assume $\left(y_{1}: y_{2}: y_{3}\right)$ and $\left(z_{1}: z_{2}: z_{3}\right)$ are the homogenous 
coordinates of two points on a line. The line equation is then given by:

$$
\operatorname{det}\left[\begin{array}{lll}
x_{1} & x_{2} & x_{3} \\
y_{1} & y_{2} & y_{3} \\
z_{1} & z_{2} & z_{3}
\end{array}\right]=0
$$

where $\left(x_{1}: x_{2}: x_{3}\right)$ is any point on the line. Using the Grassmann expansion [12] we have:

$$
\operatorname{det}\left[\begin{array}{ll}
y_{2} & y_{3} \\
z_{2} & z_{3}
\end{array}\right] x_{1}+\operatorname{det}\left[\begin{array}{ll}
y_{3} & y_{1} \\
z_{3} & z_{1}
\end{array}\right] x_{2}+\operatorname{det}\left[\begin{array}{ll}
y_{1} & y_{2} \\
z_{1} & z_{2}
\end{array}\right] x_{3}=0 .
$$

The line coordinates are therefore given by:

$$
\left(L_{1}: L_{2}: L_{3}\right)=\left(\operatorname{det}\left[\begin{array}{ll}
y_{2} & y_{3} \\
z_{2} & z_{3}
\end{array}\right]: \operatorname{det}\left[\begin{array}{ll}
y_{3} & y_{1} \\
z_{3} & z_{1}
\end{array}\right]: \operatorname{det}\left[\begin{array}{ll}
y_{1} & y_{2} \\
z_{1} & z_{2}
\end{array}\right]\right) .
$$

Similarly, since points and lines in the plane are dual elements, then two distinct lines determine exactly one point. If $\left(B_{1}: B_{2}: B_{3}\right)$ and $\left(C_{1}: C_{2}: C_{3}\right)$ are distinct lines in $P^{2}(\mathbb{R})$, then we have:

$$
\operatorname{det}\left[\begin{array}{lll}
L_{1} & L_{2} & L_{3} \\
B_{1} & B_{2} & B_{3} \\
C_{1} & C_{2} & C_{3}
\end{array}\right]=0
$$

where $\left(L_{1}: L_{2}: L_{3}\right)$ is any line through the intersection point. Using Grassmann expansion we get:

$$
\operatorname{det}\left[\begin{array}{ll}
B_{2} & B_{3} \\
C_{2} & C_{3}
\end{array}\right] L_{1}+\operatorname{det}\left[\begin{array}{ll}
B_{1} & B_{3} \\
C_{1} & C_{3}
\end{array}\right] L_{2}+\operatorname{det}\left[\begin{array}{ll}
B_{1} & B_{2} \\
C_{1} & C_{2}
\end{array}\right] L_{3}=0
$$

Hence, the point coordinates are given by:

$$
\left(x_{1}: x_{2}: x_{3}\right)=\left(\operatorname{det}\left[\begin{array}{ll}
B_{2} & B_{3} \\
C_{2} & C_{3}
\end{array}\right]: \operatorname{det}\left[\begin{array}{ll}
B_{1} & B_{3} \\
C_{1} & C_{3}
\end{array}\right]: \operatorname{det}\left[\begin{array}{ll}
B_{1} & B_{2} \\
C_{1} & C_{2}
\end{array}\right]\right) \text {. }
$$

\subsection{The Grünwald-Blaschke Mapping}

The Grünwald-Blaschke Mapping is a planar kinematic mapping and can be considered as a special case of Study's Mapping which maps the set $S E(3)$ into 
$P^{7}(\mathbb{R})$ [11]. The Grünwald-Blaschke Mapping maps the set $S E(2)$ onto points in a 3-dimensional projective image space $P^{3}(\mathbb{R})$. The general planar displacement for a moving frame $E$ with respect to a fixed frame $\Sigma$, as shown in Chapter 2, is given by:

$$
X=A x+d
$$

where in $\mathbb{R}^{3}$,

$$
A=\left[\begin{array}{cc}
\cos \phi & -\sin \phi \\
\sin \phi & \cos \phi
\end{array}\right] \in S O(2), \quad d=\left[\begin{array}{l}
a \\
b
\end{array}\right] \in \mathbb{R}^{2} .
$$

The rotation matrix $A$ can be rewritten in another nice form by using Cayley's formula as follows [1]:

$$
A=(I-B)^{-1}(I+B)
$$

where $B$ is any skew matrix. To demonstrate that such an $A$ is orthogonal, we have:

$$
(I-B) A=(I+B)
$$

Note that $(I+B)(I-B)=(I-B)(I+B)=I-B^{2}$, so that the matrices commute.

By taking the transpose of both sides of $(I-B) A=(I+B)$ :

$A^{T}(I-B)^{T}=(I+B)^{T}$

$\Leftrightarrow A^{T}\left(I^{T}-B^{T}\right)=I^{T}+B^{T}$ by properties of transpose

$\Leftrightarrow A^{T}(I+B)=I-B \quad$ since $\quad B^{T}=-B$

$\Leftrightarrow A^{T}=(I-B)(I+B)^{-1} \quad$ assuming $\quad I+B \quad$ is invertible i.e. $\quad \operatorname{det}(I+B) \neq 0$.

Now we have,

$$
\begin{aligned}
& A A^{T}=(I-B)^{-1}(I+B)(I-B)(I+B)^{-1} \\
& =(I-B)^{-1}(I-B)(I+B)(I+B)^{-1} \\
& =I .
\end{aligned}
$$


Then, the orthogonality condition $A A^{T}=I$ is fulfilled and $A \in O(n)$ by Theorem 2.1.2. Now we show $A \in S O(n)$ by checking its determinant as follows:

$$
\begin{aligned}
& A=(I-B)^{-1}(I+B) \\
& \Rightarrow \operatorname{det} A=\operatorname{det}(I-B)^{-1} \operatorname{det}(I+B) \\
\text { Now, } & \operatorname{det}(I+B)=\operatorname{det}\left(I-B^{T}\right)=\operatorname{det}(I-B)^{T}=\operatorname{det}(I-B) \quad \text { then, } \\
& \operatorname{det} A=\frac{\operatorname{det}(I+B)}{\operatorname{det}(I-B)}=\frac{\operatorname{det}(I-B)}{\operatorname{det}(I-B)}=1
\end{aligned}
$$

thus, $A \in S O(n)$. In particular when $n=2, A \in S O(2)$. The skew matrix $B$ is expressed as

$$
B=\left[\begin{array}{cc}
0 & -\alpha \\
\alpha & 0
\end{array}\right]
$$

If we let $\alpha=\tan \frac{\phi}{2},-\pi<\phi<\pi$, then an orthogonal matrix $A$ can be written explicitly in terms of the tangent of half the rotation angle:

$$
\begin{gathered}
A=(I-B)^{-1}(I+B) \\
=\left(\left[\begin{array}{ll}
1 & 0 \\
0 & 1
\end{array}\right]-\left[\begin{array}{cc}
0 & -\alpha \\
\alpha & 0
\end{array}\right]\right)^{-1}\left[\begin{array}{cc}
1 & -\alpha \\
\alpha & 1
\end{array}\right] \\
=\left[\begin{array}{cc}
1 & \alpha \\
-\alpha & 1
\end{array}\right]^{-1}\left[\begin{array}{cc}
1 & -\alpha \\
\alpha & 1
\end{array}\right] \\
=\frac{1}{1+\alpha^{2}}\left[\begin{array}{cc}
1 & -\alpha \\
\alpha & 1
\end{array}\right]\left[\begin{array}{cc}
1 & -\alpha \\
\alpha & 1
\end{array}\right] \\
=\frac{1}{1+\alpha^{2}}\left[\begin{array}{cc}
1-\alpha^{2} & -2 \alpha \\
2 \alpha & 1-\alpha^{2}
\end{array}\right] \\
=\Delta^{-1}\left[\begin{array}{cc}
1-\alpha^{2} & -2 \alpha \\
2 \alpha & 1-\alpha^{2}
\end{array}\right]
\end{gathered}
$$

where $\Delta=1+\alpha^{2}=1+\tan ^{2} \frac{\phi}{2}$ and it tells us how the parameter $\alpha$ interpreted geometrically [1]. We exclude $\phi=\pi$ for the time being to avoid points at infinity. Now if we let $\alpha=\frac{u}{v}$ with $v \neq 0$ (as we so far exclude $\phi=\pi$ ), then

$$
A=\frac{1}{1+\frac{u^{2}}{v^{2}}}\left[\begin{array}{cc}
1-\frac{u^{2}}{v^{2}} & -2 \frac{u}{v} \\
2 \frac{u}{v} & 1-\frac{u^{2}}{v^{2}}
\end{array}\right]
$$


$=\frac{1}{\frac{v^{2}+u^{2}}{v^{2}}}\left[\begin{array}{cc}\frac{v^{2}-u^{2}}{v^{2}} & \frac{-2 u}{v} \\ \frac{2 u}{v} & \frac{v^{2}-u^{2}}{v^{2}}\end{array}\right]=\frac{1}{v^{2}+u^{2}}\left[\begin{array}{cc}v^{2}-u^{2} & -2 u v \\ 2 u v & v^{2}-u^{2}\end{array}\right]=N^{-1}\left[\begin{array}{cc}v^{2}-u^{2} & -2 u v \\ 2 u v & v^{2}-u^{2}\end{array}\right]$ where $N=v^{2}+u^{2}[15]$. Therefore, a planar Euclidean isometry in terms of the three characteristic parameters $(a, b, \phi)$ can be converted to homogenous coordinates, and the exclusion case disappears. The linear homogenous transformation is then:

$$
\left[\begin{array}{l}
X \\
Y \\
Z
\end{array}\right]=\left[\begin{array}{ccc}
\frac{v^{2}-u^{2}}{N} & \frac{-2 u v}{N} & a \\
\frac{2 u v}{N} & \frac{v^{2}-u^{2}}{N} & b \\
0 & 0 & 1
\end{array}\right]\left[\begin{array}{l}
x \\
y \\
z
\end{array}\right]
$$

Multiplying by $\mathrm{N}$ we get

$$
\left[\begin{array}{l}
X \\
Y \\
Z
\end{array}\right]=\left[\begin{array}{ccc}
v^{2}-u^{2} & -2 u v & a\left(v^{2}+u^{2}\right) \\
2 u v & v^{2}-u^{2} & b\left(v^{2}+u^{2}\right) \\
0 & 0 & v^{2}+u^{2}
\end{array}\right]\left[\begin{array}{l}
x \\
y \\
z
\end{array}\right]
$$

since, for a homogenous coordinates $(N X: N Y: N Z)=(X: Y: Z)$. The matrix form of the transformation

$$
\left[\begin{array}{ccc}
v^{2}-u^{2} & -2 u v & a\left(v^{2}+u^{2}\right) \\
2 u v & v^{2}-u^{2} & b\left(v^{2}+u^{2}\right) \\
0 & 0 & v^{2}+u^{2}
\end{array}\right]
$$

indeed represents an element of $S E(2)$ and the ratios $(x: y: z)$ represent the homogeneous coordinates of a point in $E$ and $(X: Y: Z)$ are those of the same point in $\Sigma$. Then the moving coordinate frame $E$ transforms from its zero position by three parameters $(a, b, \phi)$ in $\Sigma$ where the point $(a, b)$ is the origin of $E$ that is measured in $\Sigma$. The rotation angle between the frames is $\phi(0 \leq \phi \leq 2 \pi)$ which is measured from the $X$-axis to the $x$-axis, and a rotation occurs around a fixed point in a plane. Then, a position of $E$ relative to $\Sigma$ is determined by the three parameters. This tells $E$ is a three-dimensional space. This gives rise to the existence of the Grünwald-Blaschke mapping for which $P^{3}(\mathbb{R})$ is its image space, with homogenous coordinates $\left(X_{1}: X_{2}: X_{3}: X_{4}\right)$. Note that since $\alpha=\tan \frac{\phi}{2}=\frac{\sin \frac{\phi}{2}}{\cos \frac{\phi}{2}}$, we may write $u=\sin \frac{\phi}{2}, v=\cos \frac{\phi}{2}$ above. 
Definition 3.2.1 The Grünwald-Blaschke mapping $S E(2) \longrightarrow P^{3}(\mathbb{R})$ is given by

$$
\left(X_{1}: X_{2}: X_{3}: X_{4}\right)=((a u-b v):(a v+b u): 2 u: 2 v),
$$

further, writing $u=\sin \frac{\phi}{2}, v=\cos \frac{\phi}{2}$,

$$
\left(X_{1}: X_{2}: X_{3}: X_{4}\right)=\left(\frac{1}{2}\left(a \sin \frac{\phi}{2}-b \cos \frac{\phi}{2}\right): \frac{1}{2}\left(a \cos \frac{\phi}{2}+b \sin \frac{\phi}{2}\right): \sin \frac{\phi}{2}: \cos \frac{\phi}{2}\right) \text {. }
$$

Consequently, the inverse of the Grünwald-Blaschke Mapping provides an algebraic description of the planar motion group $S E$ (2). Rewriting Eq.(3.10) in terms of the image space coordinates $\left(X_{i}\right), i=1, \ldots, 4$ [7]:

From Grünwald-Blaschke Mapping Eq.(3.11):

$$
\begin{aligned}
& X_{1}=a u-b v \\
& X_{2}=a v+b u \\
& X_{3}=2 u \\
& X_{4}=2 v,
\end{aligned}
$$

then, solving these equations simultaneously we obtain the inverse:

$$
\begin{aligned}
b & =\frac{2\left(X_{3} X_{2}-X_{1} X_{4}\right)}{X_{4}^{2}+X_{3}^{2}} \\
a & =\frac{2\left(X_{3} X_{1}+X_{4} X_{2}\right)}{X_{3}^{2}+X_{4}^{2}} \\
\tan \frac{\phi}{2} & =\frac{X_{3}}{X_{4}} .
\end{aligned}
$$

Substituting these values in Eq.(3.13) into Eq.(3.10) and then multiplying it by 4, since the transformation is homogenous, we obtain:

$$
\left[\begin{array}{c}
X \\
Y \\
Z
\end{array}\right]=\left[\begin{array}{ccc}
X_{4}^{2}-X_{3}^{2} & -2 X_{3} X_{4} & 2\left(X_{1} X_{3}+X_{2} X_{4}\right) \\
2 X_{3} X_{4} & X_{4}^{2}-X_{3}^{2} & 2\left(X_{2} X_{3}-X_{1} X_{4}\right) \\
0 & 0 & X_{3}^{2}+X_{4}^{2}
\end{array}\right]\left[\begin{array}{l}
x \\
y \\
z
\end{array}\right]
$$


The Grünwald-Blaschke Mapping is an injective map but not surjective, because a point in the image space with the coordinate $\left(X_{1}, X_{2}, 0,0\right)$ does not have a pre-image identified by the displacement parameters $(a, b, \phi)$. This is because the parameters $a, b$ will tend to infinity if $X_{3}=X_{4}=0$ and leave $\phi$ undefined, hence producing a non real displacement of $\mathrm{E}$ which does not concern us here. Therefore, a condition must be satisfied on the image space coordinates, namely $X_{3}$ and $X_{4}$ are not both zero in order to obtain images of real displacements.

\subsubsection{The inverse of the homogenous displacement}

The matrix associated with the homogenous transformation represents an element of $S E(2)$ as mentioned which leads to the existence of its inverse since $S E(2)$ is a group. Hence, the homogenous transformation may be rewritten

$$
\left[\begin{array}{l}
x \\
y \\
z
\end{array}\right]=\left[\begin{array}{ccc}
X_{4}^{2}-X_{3}^{2} & 2 X_{3} X_{4} & 2\left(X_{1} X_{3}-X_{2} X_{4}\right) \\
-2 X_{3} X_{4} & X_{4}^{2}-X_{3}^{2} & 2\left(X_{2} X_{3}+X_{1} X_{4}\right) \\
0 & 0 & X_{3}^{2}+X_{4}^{2}
\end{array}\right]\left[\begin{array}{c}
X \\
Y \\
Z
\end{array}\right] .
$$

Then, from Eq.(3.14) the coordinates of a point $(x: y: z)$ in the moving frame has coordinates $(X: Y: Z)$ in the fixed frame:

$$
\begin{aligned}
& X=\left(X_{4}^{2}-X_{3}^{2}\right) x-\left(2 X_{3} X_{4}\right) y+2\left(X_{1} X_{3}+X_{2} X_{4}\right) z \\
& Y=\left(2 X_{3} X_{4}\right) x+\left(X_{4}^{2}-X_{3}^{2}\right) y+2\left(X_{2} X_{3}-X_{1} X_{4}\right) z \\
& Z=\left(X_{3}^{2}+X_{4}^{2}\right) z
\end{aligned}
$$

whereas the coordinates of a point $(X: Y: Z)$ in the moving frame from Eq.(3.15) has coordinates $(x: y: z)$ in the fixed frame which are given by:

$$
\begin{aligned}
& x=\left(X_{4}^{2}-X_{3}^{2}\right) X+\left(2 X_{3} X_{4}\right) Y+2\left(X_{1} X_{3}-X_{2} X_{4}\right) Z, \\
& y=-\left(2 X_{3} X_{4}\right) X+\left(X_{4}^{2}-X_{3}^{2}\right) Y+2\left(X_{2} X_{3}+X_{1} X_{4}\right) Z, \\
& z=\left(X_{3}^{2}+X_{4}^{2}\right) Z
\end{aligned}
$$

Eq.(3.16) and Eq.(3.17) are algebraic inverse and they are required for the kinematic inversion that is introduced in Chapter 5 [8]. 


\section{Chapter 4}

\section{Gröbner Bases}

The purpose of this chapter is to introduce an algebraic tool of Gröbner bases and an important result, Bezout's Theorem. These are valuable in many problems in algebraic geometry and non linear computational geometry, in particular for our main problem. Polynomials provide a relation between algebra and geometry. The set of polynomials in $n$ variables with coefficients in a field $k$ is denoted $k\left[x_{1}, \ldots, x_{n}\right]$. It is a ring under polynomial addition and multiplication but it fails to be a field because in general a polynomial has no multiplicative inverse in $k\left[x_{1}, \ldots, x_{n}\right]$. Polynomials give rise on the one hand to geometric structure called affine varieties, and on the other hand algebraic structure called ideals. A brief discussion will cover both of these structures. This will lead us to our useful tool Gröbner bases which are produced by an algorithm that transforms a given polynomial set in a problem into Gröbner basis form. Ordering the terms in a polynomial in a standard way is the key to obtain the Gröbner basis form. These terms are called monomials which have the form $x^{\alpha}=x_{1}^{\alpha_{1}} \cdot x_{2}^{\alpha_{2}} \ldots x_{n}^{\alpha_{n}}$ where $\alpha=\left(\alpha_{1}, \ldots, \alpha_{n}\right) \in \mathbb{Z}_{\geq 0}^{n}$ and $\mathbb{Z}_{\geq 0}=\{0,1,2, \ldots\}$ the set of natural numbers. The total degree of this monomial is $|\alpha|=\sum_{i=1}^{n} \alpha_{i}$. Then, a polynomial $f\left(x_{1}, \ldots, x_{n}\right)$ with coefficients in $k$ is a finite linear combination of monomials and it is written:

$$
f=\sum_{\alpha \in \mathbb{Z}_{\geq 0}^{n}} c_{\alpha} x^{\alpha}, \quad c_{\alpha} \in k
$$




\subsection{Affine Varieties and Ideals}

We follow [3] to define an affine variety $V$ and an ideal $I$ :

Definition 4.1.1 Let $k$ be a field and $f_{1}, \ldots, f_{s}$ be polynomials in $k\left[x_{1}, \ldots, x_{n}\right]$. Then, an affine variety $V$ defined by $f_{1}, \ldots, f_{s}$ is

$$
V\left(f_{1}, \ldots, f_{s}\right)=\left\{\left(a_{1}, \ldots, a_{n}\right) \in k^{n}: f_{i}\left(a_{1}, \ldots, a_{n}\right)=0 \quad \forall \quad 1 \leq i \leq s\right\}
$$

where $k^{n}=\left\{\left(a_{1}, \ldots, a_{n}\right): a_{1}, \ldots, a_{n} \in k\right\}$ is the $n$-dimensional affine space.

Definition 4.1.2 An ideal $I \subset k\left[x_{1}, \ldots, x_{n}\right]$ satisfies the following:

(1) $0 \in I$

(2) If $f, g \in I$, then $f+g \in I$

(3) If $f \in I$ and $h \in k\left[x_{1}, \ldots, x_{n}\right]$, then $h f \in I$.

We denote the ideal generated by polynomials $f_{1}, \ldots, f_{s} \in k\left[x_{1}, \ldots, x_{n}\right]$ by $\left\langle f_{1}, \ldots, f_{s}\right\rangle$ and it is defined:

Definition 4.1.3 The ideal generated by $f_{1}, \ldots, f_{s} \in k\left[x_{1}, \ldots, x_{n}\right]$ is the set of all elements of the form

$$
\left\langle f_{1}, \ldots, f_{s}\right\rangle=\left\{\sum_{i=1}^{s} h_{i} f_{i}: h_{1}, \ldots, h_{s} \in k\left[x_{1}, \ldots, x_{n}\right]\right\} .
$$

In the case $I=\left\langle f_{1}, \ldots, f_{s}\right\rangle$ we say $I$ is finitely generated and $\left\{f_{i}, i=1, \ldots, s\right\}$ is a basis for $I$. There are may be many different bases for an ideal $I$. This leads us to the Hilbert Basis Theorem and a Gröbner basis that will be presented later in this chapter. Before introducing them we need to first define monomial ordering. 


\subsection{Monomial Ordering}

The only way that monomials in a single variable can be sensibly ordered is by their degrees. In the multivariable case, the situation is more complicated and the definition of monomial ordering gives the acceptable features to consider to order these monomials.

Definition 4.2.1 A relation $>$ on the set of monomials $\left\{x^{\alpha}: \alpha \in \mathbb{Z}_{\geq 0}^{n}\right\}$, or equivalently on $\mathbb{Z}^{n}$ itself is called a monomial ordering on $k\left[x_{1}, \ldots, x_{n}\right]$ if it obeys:

(1) $>$ is a total ordering.

(2) For any $\gamma \in \mathbb{Z}^{n}$, if $x^{\alpha}>x^{\beta}$, then $x^{\alpha} x^{\gamma}>x^{\beta} x^{\gamma}$.

(3) $>$ is well ordering. This means that there is a least element under $>$ for every non-empty set of monomials in $k\left[x_{1}, \ldots, x_{n}\right]$.

Some examples of monomial orderings with the necessary properties are:

(1) Lexicographic Order: Let $\alpha=\left(\alpha_{1}, \ldots, \alpha_{n}\right)$ and $\beta=\left(\beta_{1}, \ldots, \beta_{n}\right) \in \mathbb{Z}^{n}$. If the left-most non-zero component in the vector difference $\alpha-\beta$ is $\alpha_{i}-\beta_{i}$ for some $i \in\{1, \ldots, n\}$, then,

$$
\alpha>_{\text {lex }} \beta \quad \text { or } \quad\left(x^{\alpha}>_{\text {lex }} x^{\beta}\right) \quad \text { if and only if } \quad \alpha_{i}-\beta_{i}>0
$$

for instance, under the assumption of $x_{1}>x_{2}>x_{3}$ in $k\left[x_{1}, x_{2}, x_{3}\right]: x_{1}^{5} x_{2} x_{3}^{4}>$ lex $x_{1}^{2} x_{2}^{8}>_{\text {lex }} x_{1} x_{2}^{4}>_{\text {lex }} x_{1} x_{2} x_{3}^{3}$.

(2) Graded Lexicographic Order: This takes account of the total degree of the monomials. Let $\alpha, \beta \in \mathbb{Z}^{n}$. If either

$$
|\alpha|>|\beta| \text { or }|\alpha|=|\beta| \text { and } \alpha>_{\text {lex }} \beta
$$


then, we say

$$
x^{\alpha}>_{\text {glex }} x^{\beta}
$$

for instance, in $k\left[x_{1}, x_{2}, x_{3}\right]: x_{1}^{5} x_{2} x_{3}^{6}>_{\text {grlex }} x_{1}^{3} x_{2}^{6}>_{\text {grlex }} x_{1}^{2} x_{2}^{4}>_{\text {grlex }} x_{1}^{2} x_{2} x_{3}^{3}$.

(3) Graded Reverse Lexicographic Order Let $\alpha, \beta \in \mathbb{Z}^{n}$. Take the right-most non-zero component in the vector difference $\alpha-\beta$ say $\alpha_{i}-\beta_{i}$ where $i \in$ $\{1, \ldots, n\}$. Then, $\alpha>_{\text {grevlex }} \beta$ or $x^{\alpha}>_{\text {grevlex }} x^{\beta}$ if either

$$
|\alpha|>|\beta| \text { or }|\alpha|=|\beta| \text { and }
$$

$\alpha_{i}-\beta_{i}<0$. For instance, $x_{1}^{2} x_{2}^{8}>_{\text {grevlex }} x_{1}^{5} x_{2} x_{3}^{4}>_{\text {grevlex }} x_{1} x_{2}^{4}>_{\text {grevlex }} x_{1} x_{2} x_{3}^{3}$. This is again under the assumption $x_{1}>x_{2}>x_{3}$ in $k\left[x_{1}, x_{2}, x_{3}\right]$.

The following proof shows that the grevlex order is a monomial order according to Definition 4.2.1:

(1) Graded Reverse Lexicographic Order $>_{\text {grevlex }}$ is a total ordering follows directly from the definition and the fact that the usual numerical order on $\mathbb{Z}_{\geq 0}$ is a total ordering, since if $\alpha \neq \beta$, then either $|\alpha|>|\beta|$ or $|\alpha|<|\beta|$, or if $|\alpha|=|\beta|$, then for some $i, \alpha_{i} \neq \beta_{i}$ and for the greatest such $i$, either $\alpha_{i}>\beta_{i}$ or $\alpha_{i}<\beta_{i}$.

(2) If $\alpha>_{\text {grevlex }} \beta$, then $|\alpha|>|\beta|$ or $|\alpha|=|\beta|$ and the right-most nonzero entry in $\alpha-\beta$, say $\alpha_{k}-\beta_{k}$, is negative. But $x^{\alpha} x^{\gamma}=x^{\alpha+\gamma}$ and $x^{\beta} x^{\gamma}=x^{\beta+\gamma}$, then we need to show $|\alpha+\gamma|>|\beta+\gamma|$ or $|\alpha+\gamma|=|\beta+\gamma|$ and the right-most nonzero entry in $(\alpha+\gamma)-(\beta+\gamma)$ is negative. Since $|\alpha+\gamma|=|\alpha|+|\gamma|$ that is shown by the proof: If $\alpha=\left(\alpha_{1}, \ldots, \alpha_{n}\right) \in \mathbb{Z}^{n}, \gamma=\left(\gamma_{1}, \ldots, \gamma_{n}\right) \in \mathbb{Z}^{n}$, then $|\alpha|=\sum_{i=1}^{n} \alpha_{i},|\gamma|=\sum_{i=1}^{n} \gamma_{i}$. This implies $|\alpha+\gamma|=\sum_{i=1}^{n}\left(\alpha_{i}+\gamma_{i}\right)=$ $\sum_{i}^{n} \alpha_{i}+\sum_{i}^{n} \gamma_{i}=|\alpha|+|\gamma|$, then $|\alpha+\gamma|=|\alpha|+|\gamma|$. When $|\alpha|>|\beta|$, then, $|\alpha+\gamma|>|\beta+\gamma|$ or when $|\alpha|=|\beta|$ and the right-most non zero entry in $\alpha-\beta$ is negative, then, $|\alpha+\gamma|=|\beta+\gamma|$, and in $(\alpha+\gamma)-(\beta+\gamma)=\alpha-\beta$, the right-most nonzero entry is again $\alpha_{k}-\beta_{k}<0$. 
(3) Suppose that $>_{\text {grevlex }}$ were not a well-ordering, then there would be an infinite strictly decending sequence of elements of $\mathbb{Z}_{\geq 0}^{n}$

$$
\alpha(1)>_{\text {grevlex }} \alpha(2)>_{\text {grevlex }} \alpha(3)>_{\text {grevlex }} \ldots,
$$

we will show that this leads to a contradiction. Consider the last entries of the vectors $\alpha(i) \in \mathbb{Z}_{\geq 0}^{n}$. By definition of grevlex order, these last entries form a non increasing sequence of negative integers. Since $\mathbb{Z}$ is well ordered, the last entries of the $\alpha(i)$ must stabilize eventually. That is, there exists a $k$ such that all the last components of the $\alpha(i)$ with $i \geq k$ are equal. Beginning at $\alpha(k)$, the the last subsequent entries come into play in determining the grevlex order. The subsequent of the last entries of $\alpha(k), \alpha(k+1), \ldots$ form a non increasing sequence. By the same reasoning, these subsequent entries stabilize eventually. Counting in the same way, we see that for some $l$, the $\alpha(l), \alpha(l+1), \ldots$ all are equal. This contradicts the fact that $\alpha(l)>\alpha(l+1)$.

These monomial orders are available to use in most computer algebra systems such as Maple. Once such an order is decided, we apply it to the terms of a given polynomial $f=\sum_{\alpha} c_{\alpha} x^{\alpha}$. Then, the multideg of $f$ with respect to the chosen order is the $\max \left(\alpha \in \mathbb{Z}_{\geq 0}^{n}: c_{\alpha} \neq 0\right)$ which is denoted multideg $(f)$. The leading term is denoted $L T(f)$ and it includes the multideg $(f)$ together with the coefficient. The leading term without the coefficient is called the leading monomial, denoted $L M(f)$. Clearly, the $L T(f)$ for a particular monomial order is unique.

The ideal of leading terms $\langle L T(I)\rangle$ for a given ideal $I \subset k\left[k_{1}, \ldots, k_{n}\right]$ is the ideal generated by the set $L T(I)=\{L T(f): f \in I\}$, namely its set of leading terms. When an ideal $I$ is finitely generated, that is $I=\left\langle f_{1}, \ldots, f_{S}\right\rangle$, this does not always give $\langle L T(I)\rangle=\left\langle L T\left(f_{1}\right), \ldots, L T\left(f_{s}\right)\right\rangle$, for example: if $I=\left\langle f_{1}, f_{2}\right\rangle$, where $f_{1}=$ $x^{3}-2 x y$ and $f_{2}=x^{2} y-2 y^{2}+x$, and use the grlex ordering on monomials in $k[x, y]$. Then,

$$
x \cdot\left(x^{2} y-2 y^{2}+x\right)-y \cdot\left(x^{3}-2 x y\right)=x^{2}
$$


which means that $x^{2} \in I$. Thus, $L T\left(x^{2}\right)=x^{2} \in\langle L T(I)\rangle$. But $x^{2}$ is not divisible by $L T\left(f_{1}\right)=x^{3}$ or $L T\left(f_{2}\right)=x^{2} y$, then $x^{2} \notin\left\langle L T\left(f_{1}\right), L T\left(f_{2}\right)\right\rangle$, which shows

$$
\langle L T(I)\rangle \not \subset\left\langle L T\left(f_{1}\right), \ldots, L T\left(f_{s}\right)\right\rangle,
$$

although it may occur and the basis of $I$ then becomes a standard basis with special properties. Such bases are called Gröbner bases and they are introduced in the following section.

\subsection{Gröbner bases and their Properties}

Definition 4.3.1 [3] With chosen monomial order, a Gröbner basis for an ideal $I \subset k\left[x_{1}, \ldots, k_{n}\right]$ is a finite subset $G=\left\{g_{1}, \ldots, g_{s}\right\} \subset I$ such that

$$
\left\langle L T\left(g_{1}\right), \ldots, L T\left(g_{s}\right)\right\rangle=\langle L T(I)\rangle
$$

Equivalently, the leading terms of any element of $I$ is divisible by one of the $L T\left(g_{i}\right)$. Gröbner bases have nice properties, one of which is a solution for the ideal membership problem which examines the membership of a polynomial $f$ in a given ideal $I$. An algorithm for solving this is obtained by calculating a remainder modulo $G$ by the multivariate division algorithm. This remainder is denoted $\bar{f}^{G}$ and it is unique when $G$ is a Gröbner basis. Sometimes $\bar{f}^{G}$ is called a normal form for $f$ with respect to $G$. If $\bar{f}^{G}=0$ the solution for the ideal membership is solved positively. One additional property is that a Gröbner basis is also a basis.

Theorem 4.3.2 (Hilbert Basis Theorem) Every ideal $I \subset k\left[x_{1}, \ldots, x_{n}\right]$ has a finite generating set. That is $I=\left\langle g_{1}, \ldots, g_{s}\right\rangle$ for some $g_{1}, \ldots, g_{s} \in I$.

The proof of this theorem [3] shows that the basis $\left\{g_{1}, \ldots, g_{s}\right\}$ for $I$ has the special property $\langle L T(I)\rangle=\left\langle L T\left(g_{1}\right), \ldots, L T\left(g_{s}\right)\right\rangle$ and hence every ideal has a Gröbner basis. 
The technique we use Gröbner bases for is algebraic variable elimination [14]. A monomial order on $k\left[x_{1}, \ldots, x_{n}\right]$ is called an elimination order if for each $i=1, \ldots, n-1$ any monomial involving $x_{1}, \ldots, x_{i}$ is greater than any monomial involving only $x_{i+1}, \ldots, x_{n}$. The lexicographic order is of this type with $\left(x_{1}>\right.$ $\left.x_{2}>\ldots>x_{n}\right)$. In any Gröbner basis for an ideal $I \subset k\left[x_{1}, \ldots, x_{n}\right]$, the elements containing only $x_{i+1}, \ldots, x_{n}$ are also a Gröbner basis for the ideal of polynomials in $I$ with that property. When a Gröbner basis is found with an elimination order, we hope it includes a univariate polynomial in the variable $x_{n}$ only. This can be solved in principle (perhaps numerically). If each consecutive equation has at least one new variable, then for each of these solutions there are values of the other variables which are determined by the other equations. These solutions of the Gröbner basis are the same as for the original system since all polynomial sets that generate the same ideal have the same set of zeroes by Definition 4.1.3.

\subsection{Buchberger's Algorithm}

It is possible to construct a Gröbner basis for an ideal $I$ from a basis not in the Gröbner basis form, by an algorithm called Buchberger's algorithm [3]. The structure of this algorithm is based on the following theorem which is sometimes called "Buchberger's S-pair criterion". To introduce this theorem, we define the following special combinations which such an algorithm is based on.

Definition 4.4.1 Let $g_{i}, g_{j} \in k\left[x_{1}, \ldots, x_{n}\right]$ be non-zero polynomials. The $\mathbf{S}$-polynomial of $g_{i}$ and $g_{j}$ is given by the combination

$$
S\left(g_{i}, g_{j}\right)=\frac{x^{\gamma}}{L T\left(g_{i}\right)} \cdot g_{i}-\frac{x^{\gamma}}{L T\left(g_{j}\right)} \cdot g_{j}
$$

where $\gamma=\max \left(\alpha_{i}, \beta_{i}\right)$ for $1 \leq i \leq n, \alpha=\operatorname{multideg}\left(g_{i}\right), \beta=\operatorname{multideg}\left(g_{j}\right)$ and $x^{\gamma}$ is the least common multiple of $\operatorname{LM}\left(g_{i}\right)$ and $\operatorname{LM}\left(g_{j}\right)$. 
Theorem 4.4.2 The set $G=\left\{g_{1}, \ldots, g_{s}\right\}$ for an ideal $I$ is called a Gröbner basis if and only if for all pairs $i \neq j, \overline{S\left(g_{i}, g_{j}\right)} G=0$ where $i \leq j-1$.

Now, we introduce Buchberger's Algorithm. Let $I=\left\langle f_{1}, \ldots, f_{s}\right\rangle$ be a polynomial ideal and fix a monomial order. A finite number of steps are involved in this algorithm. They are:

(Input) $G^{\prime}=\left\{f_{1}, \ldots, f_{s}\right\}$ is the given initial finite subset of $k\left[x_{1}, \ldots, x_{n}\right]$.

(Processing) We select $f_{i}, f_{j} \in G^{\prime}$ with $i \leq j-1$ and find $S\left(f_{i}, f_{j}\right)$ for all $1 \leq i \leq j \leq$ $s$. Then, calculate the remainder of $S\left(f_{i}, f_{j}\right)$ modulo $G^{\prime}$ and take it to be $\overline{S\left(f_{i}, f_{j}\right)}{ }^{\prime}=h^{\prime}$. Let $\mathscr{H}$ be the set of all non-zero $h^{\prime}$. If $\mathscr{H}=\emptyset$, then the algorithm stops. Alternatively, $G=G^{\prime} \cup \mathscr{H}$ and repeat the step.

(Output) The union of initial set $G^{\prime}$ and the set of nonzero normal forms $\mathscr{H}$ which form a Gröbner basis.

Theorem 4.4.3 Let $I=\left\langle f_{1}, \cdots, f_{s}\right\rangle \neq\{0\}$ be a polynomial ideal. Then a Gröbner basis for I is constructed in a finite number of steps.

The set of Gröbner bases $G$ may contain a generator $p$ such that $L T(p) \notin\langle L T(G-$ $\{p\})\rangle$. In that case a Gröbner basis is minimal.

Now we introduce our second task in this chapter, Bezout's theorem:

\subsection{Bezout's Theorem}

Bezout's theorem focuses on relating the number of points of intersection of two curves in $P^{2}(\mathbb{C})$ to the degrees of their reduced defining equations [3]. 
Proposition 4.5.1 The irreducible factors of a nonzero homogenous polynomial $f \in \mathbb{C}[x, y, z]$ are also homogenous, and we write:

$$
f=f_{1}^{a_{1}} \ldots f_{s}^{a_{s}}
$$

where $f_{i}$ s are irreducible factors with $f_{i}$ is not a constant multiple of $f_{j}$ for $i \neq j$, and then

$$
V(f)=V\left(f_{1}\right) \cup \ldots \cup V\left(f_{s}\right)
$$

is the minimal decomposition of $V(f)$ into irreducible components in $P^{2}(\mathbb{C})$ [3]. In addition,

$$
I(V(f))=\left\langle f_{1}, \ldots, f_{s}\right\rangle
$$

This proposition shows that every curve $C \subset P^{2}(\mathbb{C})$ has a best defining equation. If we assume $C-V(f)=0$ which implies that $I(C)=\left\langle f_{1}, \ldots, f_{s}\right\rangle$, then, $f_{1} \ldots f_{s}=0$ is the reduced defining equation for the variety $C \subset P^{2}(\mathbb{C})$.

Definition 4.5.2 Let $f$ and $g \in k[x]$ are polynomials of positive degrees

$$
\begin{aligned}
& f=a_{m} x^{m}+a_{m-1} x^{m-1}+\ldots+a_{0}=0, \quad a_{m} \neq 0 \\
& g=b_{n} x^{n}+b_{n-1} x^{n-1}+\ldots+b_{0}=0, \quad b_{n} \neq 0 .
\end{aligned}
$$

The resultant of $f$ and $g$ with respect to $x$ is defined as the $(m+n) \times(m+n)$ determinant

$$
\operatorname{Res}(f, g, x)=\operatorname{det}\left[\begin{array}{cccccccc}
a_{m} & a_{m-1} & \ldots & a_{0} & 0 & 0 & \ldots & 0 \\
0 & a_{m} & a_{m-1} & \ldots & a_{0} & 0 & \ldots & 0 \\
0 & 0 & \ddots & \ddots & \ldots & \vdots & \vdots & 0 \\
0 & 0 & \ldots & 0 & a_{m} & a_{m-1} & \ldots & a_{0} \\
b_{n} & b_{n-1} & \ldots & b_{0} & 0 & 0 & \ldots & 0 \\
0 & b_{n} & b_{n-1} & \ldots & b_{0} & 0 & \ldots & 0 \\
0 & 0 & \ddots & \ddots & \ldots & \vdots & \vdots & 0 \\
0 & 0 & \ldots & 0 & b_{n} & b_{n-1} & \ldots & b_{0}
\end{array}\right] .
$$


Lemma 4.5.3 [3] Suppose $f, g \in \mathbb{C}[x, y, z]$ are homogenous polynomials of total degree $m, n$ respectively. If $f(0,0,1)$ and $g(0,0,1)$ are nonzero, then the resultant $\operatorname{Res}(f, g, z)$ is homogenous in $x$ and $y$ of total degree $m n$.

Definition 4.5.4 Let $C$ and $D$ be curves in $P^{2}(\mathbb{C})$ without common factors and their reduced defining equations are $f=0$ and $g=0$ respectively. Choose coordinates for $P^{2}(\mathbb{C})$ such that

$$
(0,0,1) \notin C \cup D \cup \underset{p \neq q}{\bigcup_{\text {in }}} L_{C \cap D} L_{p q}
$$

where $L_{p q}$ is the projective line connecting points $p$ and $q$ in $P^{2}(\mathbb{C})$ with $p \neq q$. Then, given $p=(u: v: w) \in C \cap D$, the intersection multiplicity $I_{p}(C, D)$ is defined to be the exponent of $v x-u y$ in the factorization of $\operatorname{Res}(f, g, z)$ [3].

The above ideas are central in Bezout's Theorem (see [3] for the proof) as follows:

Theorem 4.5.5 (Bezout's Theorem). Let $C$ and $D$ be curves in $P^{2}(\mathbb{C})$ without common factors, and let the degrees of their reduced defining equations be $m$ and n. Then,

$$
\sum_{p \in C \cap D} I_{p}(C, D)=m n
$$

Furthermore, Bezout's Theorem generalizes to the projective space $P^{n}(\mathbb{C})$ [17]. This means that the number of solutions of a system of $n$ homogenous equations in $n+1$ unknowns is either infinite or equal to the product of the degrees, namely their solutions are counted with their multiplicities. 


\section{Chapter 5}

\section{Constraint Equations}

This chapter presents the application of the Grünwald-Blaschke mapping and the method of Gröbner basis to find the univariate polynomials for the fully parallel symmetric GP3LP in the forward kinematic problem. In order to define a GP3LP, we first introduce some of the necessary terminology, as follows $[9,10]$ :

(1) A collection of mechanical parts without any relative motion between individual parts is called a link.

(2) Any two links can be connected together by a mechanical constraints that allow relative motion. This relative constraint motion is described by a number of free parameters. Such mechanical constraints are called joints and such a number of free parameters is called the degree of freedom of the joint. There are in general two kinds of joint: lower pairs which are classified into six types and higher pairs. They distinguish by the type of contact. Lower pairs are determined by contact between surfaces whereas higher pairs may allow line or point contact. As planar motion is our concern, only two types of the lower pairs are planar: revolute joint which is denoted by $(R)$ and prismatic joint which is denoted by $(P)$. Each of them has one degree of freedom: rotation about a fixed point in the plane and translation in a fixed direction respectively. 
(3) A set of links connected by joints is called kinematic chain. A kinematic chain can be represented by a graph, where a graph consists of two finite sets: a set of vertices and a set of edges and edges correspond to pairs of vertices. Then, a kinematic chain consists of two finite sets: a set of links represent the vertices of the graph and a set of joints represent the edges. There are two types of kinematic chain: simple kinematic chain and complex kinematic chain. If each link is joined to at most two other links, then the chain is simple and it can be open or closed. If a simple chain contains a cycle, namely, every link in the chain joined to two other links, then it is closed. Alternately, a simple chain is open if it does not have a cycle, that is the first link and the last link in the chain are joined to just one link. However, a chain is said to be complex if at least one of its links is joined to three or more links. A complex chain can be decomposed into simple kinematic chains called sub-chains.

(4) A mechanism is the fundamental object in computational kinematics [11]. It is defined as a kinematic chain where one of the links is fixed to the fixed frame coordinate system and certain joints are identified as actuated. That is, motion of these joints is directly controlled. If the relative motion of the links occurs in one plane or parallel planes, then the mechanism is called $a$ planar mechanism.

(5) We described the DOF in Chapter 1. Here we give the formula that gives the total DOF (mobility) for a manipulator which is given by ChebychevGrübler-Kutzbach's formula [4]:

$$
\mu=n(l-1)-\sum_{i=1}^{k}\left(n-\delta_{i}\right)=\sum_{i=1}^{k} \delta_{i}-n(k-l+1)
$$

in which $\mu$ is the mobility, or relative degrees of freedom of the platform, $n$ is the number of degrees of freedom of an independent kinematic chain ( $n=3$ for planar, $n=6$ for spatial), $k$ is the number of joints, $l$ the number of links and $\delta_{i}$ the DOF of the $i$ th joint. This formula may give the wrong 
mobility in some special cases for example, when special design parameters in a manipulator act irregularly, see [4].

(6) If a mechanism consists of an open kinematic chain, then it is serial mechanism, namely the joints are built in a series, while a mechanism contains a closed kinematic chain is called parallel mechanism.

\subsection{GP3LP}

A general planar 3-legged platform (GP3LP) is constituted of a platform in a triangular shape moving in 2-dimension connected to a fixed frame of coordinate system via three simple open kinematic chains; see Fig. 5.1. Each of the three kinematic chains are formed by three independent one DOF joints confined to be either a prismatic $(\mathrm{P})$ or a revolute $(\mathrm{R})$ joint. One of them is actuated and the other two joints are free to move. These features of the joints have an effect in controlling and constraint of the motion of the moving platform which occurs with respect to the independent open kinematic chains. Therefore, each independent kinematic chain has 3 DOF. The three kinematic chains are considered as the three legs of the platform. In addition, the GP3LP is a complex chain since the platform connects to three links and each leg is a simple sub-chain. As a consequence, each of the three kinematic chain is denoted by a set of three letters indicating the sequence of the joints beginning from the fixed frame which leads to the following eight possible combination of R- and P-pairs [13]:

$$
R R R, R P R, R R P, R P P, P R R, P P R, P R P, P P P .
$$

The purpose of these leg architectures is to allow full translation and the rotation of the platform for each leg, that is to obtain three DOF. Applying the ChebychevGrübler-Kutzbach's formula to the platform in Fig. 5.1, we have $n=3, l=8$ 


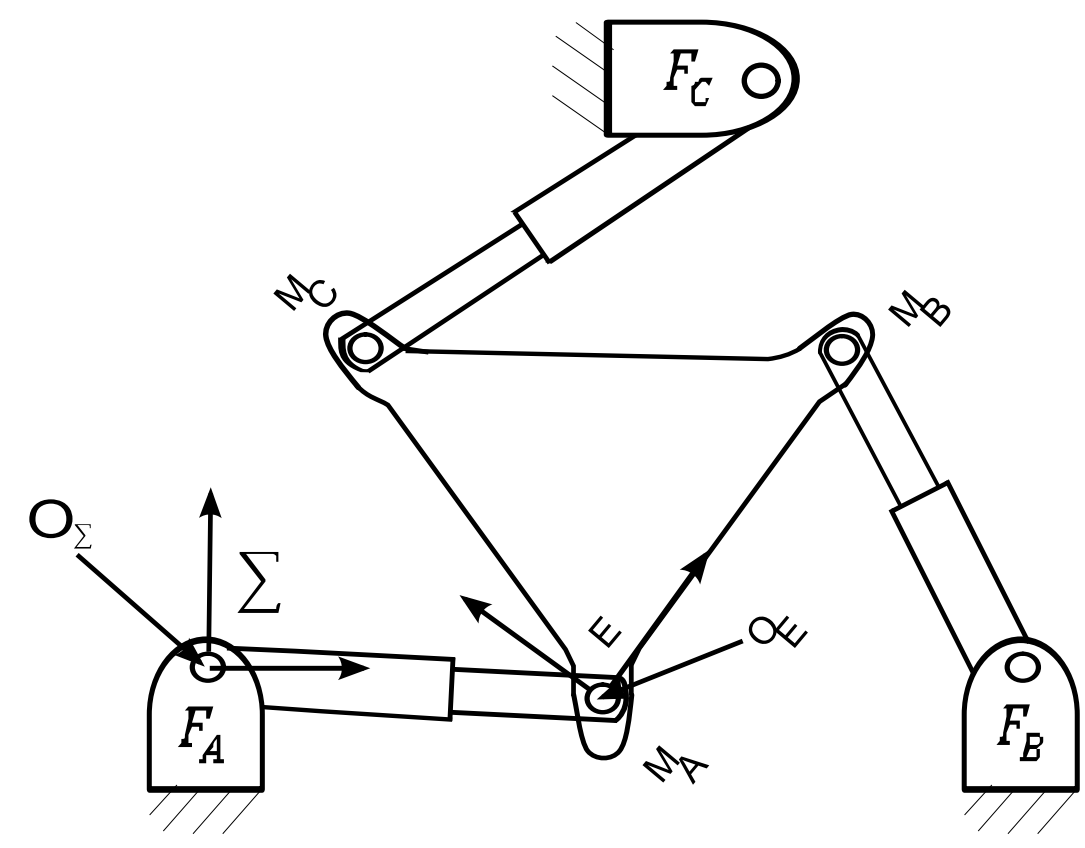

Figure 5.1: A platform with three RPR legs [8]

(including the base), $\delta_{i}=1$ for all $i=1, \ldots, 9, k=9$, so

$$
\begin{aligned}
\mu & =3(8-1)-\sum_{i=1}^{9}(3-1) \\
& =21-18 \\
& =3 .
\end{aligned}
$$

Note, there is one of the 8 combination that must be removed which is PPP since it can not give rise to any rotation, only to translations. Therefore, seven possible kinematic chains are possible and these are illustrated in Fig. 5.2. 

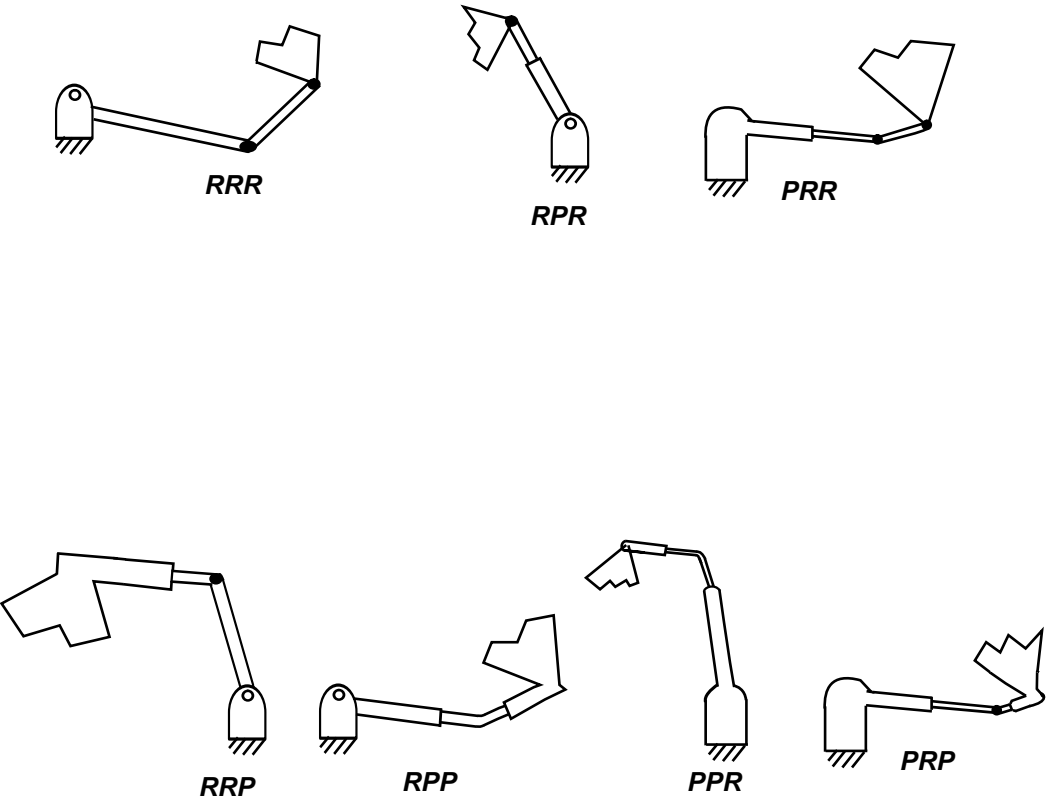

Figure 5.2: The seven possible leg topologies [8].

\subsubsection{The Passive Sub-Chain}

In order to achieve three DOF it is only necessary to have control of 3 joints. In practice, one joint in each leg is activated, that is connected to some drive mechanism. The other joints are described as passive. The actuated joint is distinguished by underlining it, for instance, $R \underline{P R}$. Hence, there are twenty-one leg architectures taking into account the active joint, as we have three choices of joint in seven possible legs. The uncontrolled motion of the leg occurs by means of the so called passive sub-chain. The formation of the passive sub-chain is caused by locking the active joint and it is effectively removed from the chain while the other two passive joints remain in the chain. As we investigate the passive sub-chains that result from the twenty-one kinematic chains, we get at most four classes. They are either RR, PR, RP or PP. The PP sub-chain is omitted as a platform with more 


\begin{tabular}{|c|c|c|}
\hline RR-Type & PR-Type & RP-Type \\
\hline RRR & RPR & RRP \\
\hline R $\underline{R R}$ & PRR & RRP \\
\hline RRR & PPR & RPR \\
\hline PRR & PPR & PRP \\
\hline RPR & $\overline{\mathrm{PPR}}$ & RPP \\
\hline RRP & PRP & RPP \\
\hline
\end{tabular}

Table 5.1: The 18 possible leg architectures.

than one such leg is not able to be assembled or controlled once the actuated joint variables are identified [9]. This exception yields a reduction in the number of the possible legs architectures from 21 to 18. They are listed in Table 5.1.

The previous description asserts there are variants of GP3LP with three DOF that can be enumerated if we consider that each of the three legs has 18 possible choices since there is a set of 18 possible kinematic chains to choose from.

\subsection{Kinematic Constraints}

This section presents the main theme of the constrained motion in the plane and the corresponding surfaces in the image space via the Grünwald-Blaschke mapping. These surfaces are called 'constraint surfaces' due to the constraint motion caused by locking the actuated joint. Initially, we determine the type of constraints for different passive sub-chains. Since a motion is a continuous set of displacements, and as a displacement transforms to a point, then a constrained motion will map to a continuous set of points in the image space [8]. Secondly, our goal is to find the equation that defines a point on a constraint surface. Thirdly, we will describe briefly the ten classes of GP3LP. Finally, we will apply the point equation to find the univariate polynomials by Gröbner basis for the symmetric GP3LP which explain the solution for the forward kinematic problem and we will examine the maximum number of real solutions for these polynomials. 


\subsubsection{The Lower-Pair Constraints}

Kinematic constraints in our case are called lower-pair constraints since they are determined by lower-pair joints. A specific leg of a GP3LP involves a particular type of the passive sub-chains: RR-type, PR-type and RP-type.

\section{RR-Type}

This passive sub-chain includes two free joints both of which are revolute (R). One of them is attached to the active joint and is fixed in the reference frame $\Sigma$, while the other one represents a point on the moving frame $E$ and is constrained to move on a circle of fixed radius and fixed centre in $\Sigma$. This constraint motion is called circular constraint.

\section{PR-Type}

Two ordered different types of joints are involved in this passive sub-chain starting from the ground (the fixed frame). The R-joint represents a point of $E$ that is bound to move on a line determined by the P-joint on the fixed frame $\Sigma$. This constrained motion is called linear constraint.

\section{RP-Type}

This passive sub-chain is the kinematic inversion of the former one [8]. This means that a line in the moving frame $E$ moves on a point in the fixed frame $\Sigma$. This can easily be seen by the duality principle. Since the motion caused by a prismatic joint is a translation, then the leg is translated with different leg lengths that intersect in a point in $\Sigma$. This is also called linear constraint. 


\subsubsection{The Constraint Surface Equations}

The constraint surface is associated with the constraint motion of a leg in GP3LP where the aforementioned passive sub-chain types cause such motion. An implicit equation of the constraint surface is introduced in this section. A line might be considered as a circle with infinite radius. The general form of a circle equation is given by

$$
\left(X-X_{c}\right)^{2}+\left(Y-Y_{c}\right)^{2}=r^{2}
$$

where $\left(X_{c}, Y_{c}\right)$ is the centre of a circle with the radius $r$. It can be rewritten

$$
X^{2}-2 X X_{c}+X_{c}^{2}+Y^{2}-2 Y Y_{c}+Y_{c}^{2}=r^{2} .
$$

Passing to homogenous coordinates by putting $X=\frac{X}{Z}, Y=\frac{Y}{Z}$ we get:

$$
\left(X^{2}+Y^{2}\right)+\left(-2 X X_{c}-2 Y Y_{c}\right) Z+\left(X_{c}^{2}+Y_{c}^{2}-r^{2}\right) Z^{2}=0
$$

which is essentially expressed as:

$$
k_{0}\left(X^{2}+Y^{2}\right)+2 k_{1} X Z+2 k_{2} Y Z+k_{3} Z^{2}=0
$$

$\left[k_{0}, k_{1}, k_{2}, k_{3}\right]$ are called circle coefficients [8], where

$$
\begin{aligned}
& k_{1}=-X_{c} \\
& k_{2}=-Y_{c} \\
& k_{3}=X_{c}^{2}+Y_{c}^{2}-r^{2}
\end{aligned}
$$

and $k_{0}$ is an arbitrary homogenous constant [8], that is employed in order to distinguish the circular constraint from the linear constraint. Hence, the form of the most general constraint is given by Eq.(5.2). Then, when $k_{0}=1$ the constraint is circular and when $k_{0}=0$ the constraint is linear. By using the linear homogenous transformation of a planar displacement described in terms of the image space points that is defined by Grünwald-Blaschke Mapping Eq.(3.14) in chapter 3 (that gives the coordinates $(X, Y, Z)$ of points in the fixed frame $\Sigma$ in terms of the points 
$(x, y, z)$ in the moving frame $E)$. Then, the image space constraint surface equation obtains when the expressions for $(X: Y: Z)$ from Eq.(3.14) are substituted into Eq.(5.2) [7]:

$$
\begin{aligned}
& \left(k_{0} z^{2}\left(X_{1}^{2}+X_{2}^{2}\right)+\frac{1}{4}\left[k_{0}\left(x^{2}+y^{2}\right)+k_{3} z^{2}-2 z\left(k_{1} x+k_{2} y\right)\right] X_{3}^{2}\right. \\
& +\frac{1}{4}\left[k_{0}\left(x^{2}+y^{2}\right)+k_{3} z^{2}+2 z\left(k_{1} x+k_{2} y\right)\right] X_{4}^{2}+\left(k_{1} z^{2}-k_{0} x z\right) X_{1} X_{3} \\
& -\left(k_{2} z^{2}+k_{0} y z\right) X_{1} X_{4}+\left(k_{2} z^{2}-k_{0} y z\right) X_{2} X_{3}+\left(k_{0} x z+k_{1} z^{2}\right) X_{2} X_{4} \\
& \left.+\left(k_{2} x z-k_{1} y z\right) X_{3} X_{4}\right)\left(\frac{1}{4}\left(X_{3}^{2}+X_{4}^{2}\right)=0\right.
\end{aligned}
$$

This equation is the equation for a point on a constraint surface that is obtained in terms of the image space coordinates $X_{i}(i=1, \ldots, 4)$. It can be simplified as the second factor is necessarily non-zero. Accordingly, we may without danger divide the expression by the non-zero factor to yield a quadric in $X_{i}$, with coefficients in terms of the circle coefficients $k_{i}$ and the homogenous coordinate $(x: y: z)$ for a point in $E$ (the attachment point of a leg to the platform). As a result, this quadric equation defines points on the constraint surface in the 3-D projective image space. Moreover, some simplifications can be applied to this equation by making some assumptions regarding the platform associated with it. These assumptions are as follows [8]:

(1) Set all the attachment points in $E$ to be on the affine plane $z=1$.

(2) It is reasonable to normalize the image space coordinates $\left(X_{1}, X_{2}, X_{3}, X_{4}\right)$ by setting $X_{4}=1$, after removing the image points associated with the platform orientation $\phi=\pi$. Thus, the image coordinates in Eq.(3.12) become:

$$
\begin{aligned}
X_{1} & \left.=\frac{1}{2}\left(\tan \left(\frac{\phi}{2}\right)\right)-b\right) \\
X_{2} & =\frac{1}{2}\left(a+b\left(\tan \frac{\phi}{2}\right)\right) \\
X_{3} & =\tan \left(\frac{\phi}{2}\right) \\
X_{4} & =1 .
\end{aligned}
$$


Consequently, the constraint surface equation can be reduced to the simplified form:

$$
\begin{aligned}
& k_{0}\left(X_{1}^{2}+X_{2}^{2}\right)+\frac{1}{4}\left[k_{0}\left(x^{2}+y^{2}\right)+k_{3}-2\left(k_{1} x+k_{2} y\right)\right] X_{3}^{2}+\frac{1}{4} \\
& {\left[k_{0}\left(x^{2}+y^{2}\right)+k_{3}+2\left(k_{1} x+k_{2} y\right)\right]+\left(k_{1}-k_{0} x\right) X_{1} X_{3}-\left(k_{2}+k_{0} y\right) X_{1}} \\
& +\left(k_{2}-k_{0} y\right) X_{2} X_{3}+\left(k_{0} x+k_{1}\right) X_{2}+\left(k_{2} x-k_{1} y\right) X_{3}=0 .
\end{aligned}
$$

\section{Circular Constraint}

The motion of a platform's leg is constrained to move on a circle (its centre and radius are fixed) when it is caused by a passive sub-chain of RR-Type so that the platform can rotate with respect to this leg when the other two legs are disconnected. The constraint surface equation is therefore obtained by setting $k_{0}=1$ in Eq.(5.5) and we get:

$$
\begin{aligned}
& \left(X_{1}^{2}+X_{2}^{2}\right)+\frac{1}{4}\left[\left(x^{2}+y^{2}\right)+k_{3}-2\left(k_{1} x+k_{2} y\right)\right] X_{3}^{2}+\frac{1}{4} \\
& {\left[\left(x^{2}+y^{2}\right)+k_{3}+2\left(k_{1} x+k_{2} y\right)\right]+\left(k_{1}-x\right) X_{1} X_{3}-\left(k_{2}+y\right) X_{1}} \\
& +\left(k_{2}-y\right) X_{2} X_{3}+\left(x+k_{1}\right) X_{2}+\left(k_{2} x-k_{1} y\right) X_{3}=0 .
\end{aligned}
$$

The following algebra will show that cross-section $X_{3}=$ constant are in fact circles. First, substitute the relation between the circle coefficients $k_{i}$ in Eq.(5.3) for $k_{3}$ into Eq.(5.6). Then, we have:

$$
\begin{aligned}
& \left(X_{1}^{2}+X_{2}^{2}\right)+\frac{1}{4}\left[\left(x^{2}+y^{2}\right)+k_{1}^{2}+k_{2}^{2}-r^{2}-2\left(k_{1} x+k_{2} y\right)\right] X_{3}^{2}+\frac{1}{4} \\
& {\left[\left(x^{2}+y^{2}\right)+k_{1}^{2}+k_{2}^{2}-r^{2}+2\left(k_{1} x+k_{2} y\right)\right]+\left(k_{1}-x\right) X_{1} X_{3}-\left(k_{2}+y\right) X_{1}} \\
& +\left(k_{2}-y\right) X_{2} X_{3}+\left(x+k_{1}\right) X_{2}+\left(k_{2} x-k_{1} y\right) X_{3}=0 .
\end{aligned}
$$

Next, we collect the terms $X_{1}$ and $X_{2}$ on the left and $X_{3}$ terms on the right. Then, complete the squares in $X_{1}$ and $X_{2}$. We acquire:

$$
\left(X_{1}-\frac{1}{2}\left[\left\{x-k_{1}\right\} X_{3}+k_{2}+y\right]\right)^{2}+\left(X_{2}-\frac{1}{2}\left[\left\{y-k_{2}\right\} X_{3}-k_{1}-x\right]\right)^{2}=\frac{r^{2}}{4}\left(1+X_{3}^{2}\right) .
$$




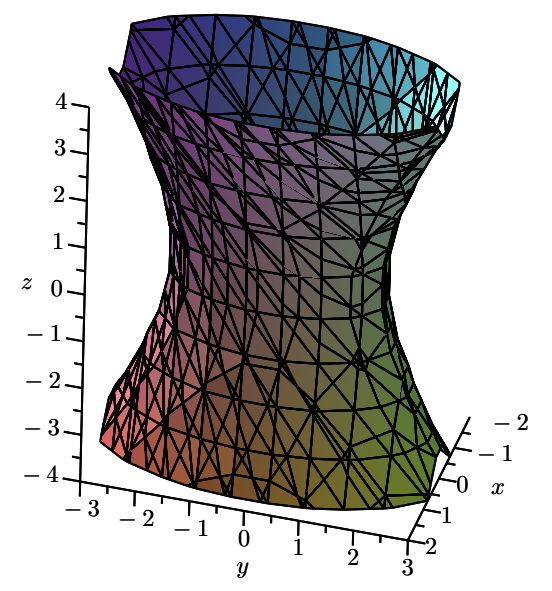

Figure 5.3: Hyperboloid of one sheet

Clearly, this represents a circle equation with the centre coordinates

$$
\left(\frac{1}{2}\left[\left\{x-k_{1}\right\} X_{3}+k_{2}+y\right]: \frac{1}{2}\left[\left\{y-k_{2}\right\} X_{3}-k_{1}-x\right]: X_{3}\right)
$$

and radius $\frac{1}{2} r \sqrt{1+X_{3}^{2}}$ [7]. This shows that cross-section $X_{3}=$ constant are circles. Then, the circle's radius vary as $X_{3}$ is varied which lead to a family of circles whose centers are collinear. Hence, this constraint variety is the family of these circles where the smallest circle occurs when $X_{3}=0$. Once the value of $X_{3}$ increases with regardless of its sign, we get larger circles and then the quadric variety extends to infinity in two directions. Hence, a quadric fulfills such properties is a hyperboloid of one sheet; see Fig. 5.3.

\section{Linear Constraint}

In the circumstance of linear constraint there are two factors that emerge when the value of $k_{0}$ in Eq.(5.2) vanishes. Then we have,

$$
Z\left(2 k_{1} X+2 k_{2} Y+k_{3} Z\right)=0
$$




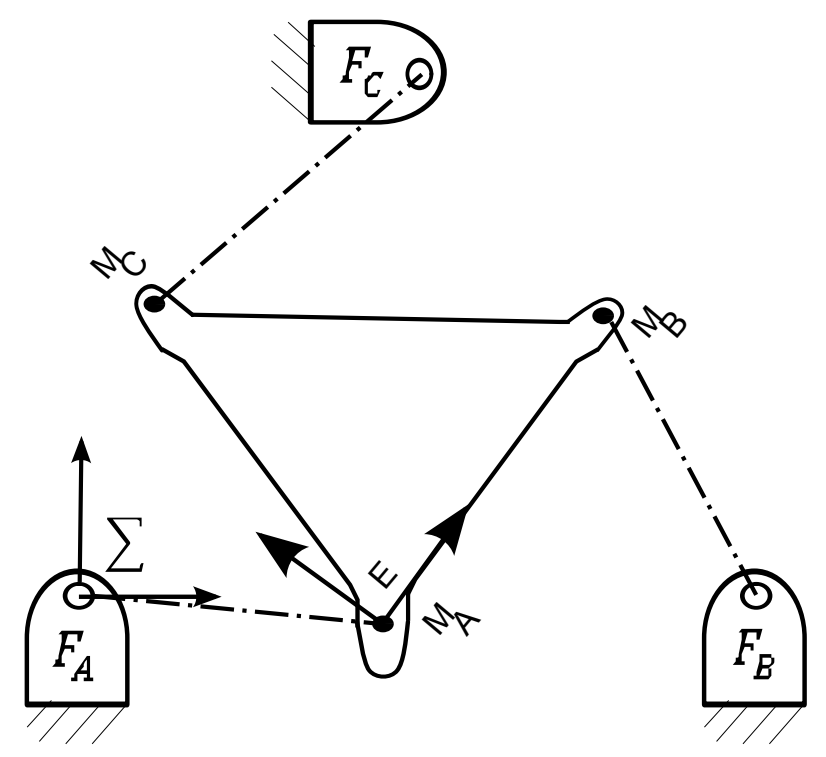

Figure 5.4: The fixed frame $\Sigma$ and the moving frame $E$ for any set of legs from Table 5.1.

Both of the factors in this equation are linear. The first factor $Z=0$ describes the line at infinity which enables us to omit this factor as it does not correspond to a real displacement. The second factor can be understood from the duality principle described in chapter 3 , that is the point $(X: Y: Z)$ determines the line $\left(2 k_{1}: 2 k_{2}: k_{3}\right)$. The line coordinates are denoted in Eq.(3.6) by $\left(L_{1}: L_{2}: L_{3}\right)$ as shown in chapter 3 , then we have:

$$
\left(2 k_{1}: 2 k_{2}: k_{3}\right)=\left(L_{1}: L_{2}: L_{3}\right)
$$

which can be rewritten to give:

$$
\left(k_{1}: k_{2}: k_{3}\right)=\left(\frac{1}{2} L_{1}: \frac{1}{2} L_{2}: L_{3}\right) .
$$

This reveals that the coefficients $k_{i}$ are equivalent to line coordinates. To determine the line coefficients we use Eq.(3.4) that specifies a line equation. Following the 
illustration in [8], and an RPR leg is used for such illustration. The line coordinates for these legs are determined by two points in the fixed frame $\Sigma$, namely by the base R-pair input and the corresponding fixed point, $F_{i}\left(X_{i}: Y_{i}: Z_{i}\right), i \in\{A, B, C\}$; see Fig. 5.4, where the base R-pair input coordinates verify the direction of the line by the angle of the actuated joint with respect to the fixed frame $\Sigma, \phi_{\Sigma}$. Since the direction of the line is a point at infinity in the projective plane, that is $z=0$, then the line equation in $\Sigma$ for the given leg is:

$$
\operatorname{det}\left[\begin{array}{ccc}
X & Y & Z \\
X_{A} & Y_{A} & Z_{A} \\
\cos \phi_{\Sigma} & \sin \phi_{\Sigma} & 0
\end{array}\right]=0
$$

which, after expansion, gives:

$$
-Z_{A} \sin \phi_{\Sigma} X+Z_{A} \cos \phi_{\Sigma} Y+\left(X_{A} \sin \phi_{\Sigma}-Y_{A} \cos \phi_{\Sigma}\right) Z=0
$$

Then, we can deduce from this equation the values of the line coefficients $k_{i}$ after applying $\left(k_{1}: k_{2}: k_{3}\right)=\left(\frac{1}{2} L_{1}: \frac{1}{2} L_{2}: L_{3}\right)$, we obtain:

$$
\left(k_{1}: k_{2}: k_{3}\right)=\left(-\frac{Z_{A}}{2} \sin \phi_{\Sigma}: \frac{Z_{A}}{2} \cos \phi_{\Sigma}:\left(X_{A} \sin \phi_{\Sigma}-Y_{A} \cos \phi_{\Sigma}\right)\right) .
$$

The constraint surface equation equipped with the linear constraint for the PR-Type leg emerges when we set $k_{0}=0$ and the values of the $k_{i}$ given above in Eq.(5.7) in the general equation for the constraint surface Eq.(5.5). Since the kinematic inversion of PR-Type leg is RP-Type, then the coefficients $k_{i}$ of the latter can be found in a similar way of PR-Type leg, though, in this case the kinematic constraint is described as a line in $E$ moves on a point in $\Sigma$. Then, the coordinates of the moving line are determined by two points in $E$, one of these points represents the homogenous coordinates $\left(x_{A}: y_{A}: z_{A}\right)$ of the revolute centre that is fixed relative to $E$ while the homogenous coordinates of the other point represent the direction of such line by the input angle of the actuated joint with respect to the moving frame $E, \phi_{E}$. Then, the line equation is defined as:

$$
\operatorname{det}\left[\begin{array}{ccc}
x & y & z \\
x_{A} & y_{A} & z_{A} \\
\cos \phi_{E} & \sin \phi_{E} & 0
\end{array}\right]=0
$$


moreover, in this case the coefficients $k_{i}$ are determined by:

$$
\left(k_{1}: k_{2}: k_{3}\right)=\left(-\frac{z_{A}}{2} \sin \phi_{E}: \frac{z_{A}}{2} \cos \phi_{E}: x_{A} \sin \phi_{E}-y_{A} \cos \phi_{E}\right)
$$

Since the homogenous linear transformation Eq.(3.14) has an inverse Eq.(3.15), then, either $(X: Y: Z)$ or $(x: y: z)$ from Eq.(3.16) or Eq.(3.17) are substituted into Eq.(5.2) the general equation of the constraint. The point equation for the most general constraint surfaces of either leg PR-Type or RP-Type is then:

$$
\begin{aligned}
& \frac{1}{4}\left[k_{3}-2\left(k_{1} x+k_{2} y\right)\right] X_{3}^{2}+k_{1} X_{1} X_{3}+k_{2} X_{2} X_{3} \\
& \mp k_{2} X_{1} \pm k_{1} X_{2} \mp\left(k_{1} y-k_{2} x\right) X_{3}+\frac{1}{4}\left(k_{3}+2\left(k_{1} x+k_{2} y\right)\right)=0
\end{aligned}
$$

where the alternate signs emerge due to the kinematic inversion that requires such algebraic inverse. In the case of PR-Type leg we substitute the values of the $k_{i}$ from equation (5.7) into Eq.(5.9) and the upper sign employ to obtain the point equation. Alternately, we substitute the values of the $k_{i}$ from equation (5.8) into Eq.(5.9), then $(X: Y: Z)$ are substituted for $(x: y: z)$ and employ the lower sign to perform the point equation for a leg of RP-Type. The constraint surface described by the linear constraint is seen to be an hyperbolic paraboloid as explained in [7], that is "Eq.(5.9) is also intersected with planes where $X_{3}$ is a constant. As $X_{3}$ is varied a family of mutually skew lines is obtained that are all parallel to a plane, but not to each other. The quadric is therefore a regulus of an hyperbolic paraboloid"; see Fig. 5.5. We conclude that the hyperboloids of one sheet and the hyperbolic paraboloids are the only surfaces corresponding to the kinematic constraints that arise from applying the kinematic mapping to a single leg of GP3LP.

\subsubsection{The Formulation of GP3LP}

The GP3LP is considered to be either symmetric when it is formulated by three legs of the same type or asymmetric alternatively. According to the three types for a passive sub-chain in a leg in GP3LP, there are 10 classes of GP3LP formed 


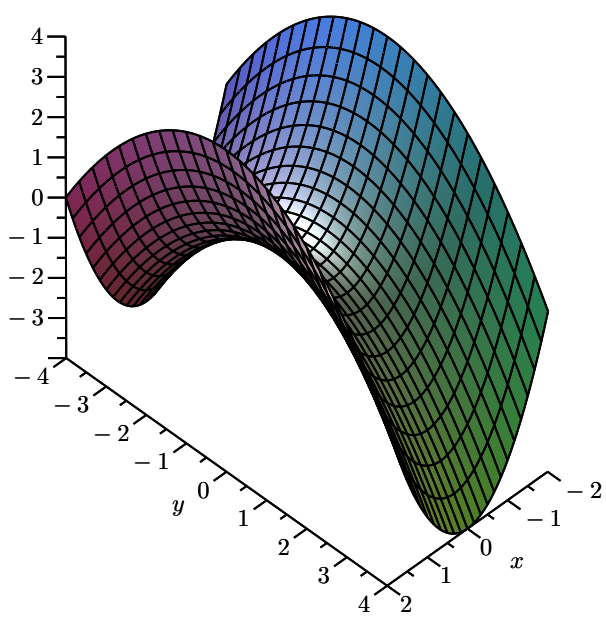

Figure 5.5: Hyperbolic Paraboloid

by both symmetric and asymmetric platforms [2]. If we use the labels established in [2], that is, we label the type RR- by $\mathrm{C}$ as it corresponds to a circular constraint, the type PR- by L since a point moves on a line and the type RP- by T since a movement occurs on a point, then, the 10 classes of GP3LP are listed in Table 5.2.

\subsection{The Forward Kinematic Problems}

Gosselin [13], was the first to analyse the forward kinematic problem of the mechanism 3-RRR, completely ( which from the forward kinematics point of view is equivalent to the 3 -RPR platform ). He obtained a univariate polynomial of degree 6 which solves this problem, by determining all the possible poses for the moving platform. The method Gosselin used was by considering the intersection between a coupler curve of the four bar mechanism with a circle where the coupler curve has degree 6 . As the number of real intersection points between the coupler curve of degree 6 and a circle is at most 6 , then, his univariate polynomial is 


\begin{tabular}{|c|c|c|c|}
\hline class of GP3LP & first leg type & second leg type & third leg type \\
\hline CCC & C & C & C \\
\hline LLL & L & L & L \\
\hline TTT & T & T & T \\
\hline CCL & C & C & L \\
\hline CCT & C & C & T \\
\hline LLT & L & L & T \\
\hline LLC & L & L & C \\
\hline TTC & T & T & C \\
\hline TTL & T & T & L \\
\hline CLT & C & L & T \\
\hline
\end{tabular}

Table 5.2: The 10 classes of GP3LP

optimum [13]. However, we solve the direct kinematics by using the method of Gröbner bases to find the univariate polynomials. This method will be applied to the symmetric platforms of the 10 formulation listed in Table 5.2. To begin with we consider the Grünwald-Blaschke Mapping that gives for each of the three legs in GP3LP a constraint surface in the projective image space. Thus, the solution for the forward kinematics in our case will be specified by the intersection points of these three surfaces in the image space. The intersection points represent the possible postures for the moving platform $E$ relative to the fixed frame $\Sigma$.

\subsubsection{C-C-C Class Legs}

A GP3LP of this class has three circular legs equations each of which is of the form Eq.(5.6). Each point with fixed coordinates in the moving frame will move on a fixed circle with a fixed centre and radius in the fixed frame. We determine first the centers of these circles. By choice of $\Sigma$, see Fig. 5.1, the first circle is centered at the origin in $\Sigma$, the second circle is centered on the $\mathrm{X}$-axis and the third one is centered on a general point with non-zero value in $\Sigma$ whereas the attachment point coordinates in the moving frame $E$ for each leg are the origin in $E$ for the first leg, for the second leg it is located on the $\mathrm{x}$-axis and a general point with non-zero 


\begin{tabular}{|c|c|c|c|}
\hline The leg & Circle center & Attachment point & Leg length \\
\hline leg 1 & $(0,0,1)$ & $(0,0,1)$ & $r_{1}$ \\
\hline leg 2 & $\left(X_{2 m}, 0,1\right)$ & $\left(x_{2}, 0,1\right)$ & $r_{2}$ \\
\hline leg 3 & $\left(X_{3 m}, Y_{3 m}, 1\right)$ & $\left(x_{3}, y_{3}, 1\right)$ & $r_{3}$ \\
\hline
\end{tabular}

Table 5.3: Point coordinates

value for the third one. These are listed in Table 5.3. Substitute these values in the circular equation (5.6) for each leg and as in [18], we get three hyperboloids of one sheet in the use of kinematic mapping. They are as follows:

$$
\begin{aligned}
& X_{1}^{2}+X_{2}^{2}+a_{6} X_{3}^{2}+a_{7}=0 \\
& X_{1}^{2}+X_{2}^{2}+b_{1} X_{1} X_{3}+b_{4} X_{2}+b_{6} X_{3}^{2}+b_{7}=0 \\
& X_{1}^{2}+X_{2}^{2}+c_{1} X_{1} X_{3}+c_{2} X_{2} X_{3}+c_{3} X_{1}+c_{4} X_{2}+c_{5} X_{3}+c_{6} X_{3}^{2}+c_{7}=0
\end{aligned}
$$

where:

$$
\begin{aligned}
& a_{6}=-\frac{r_{1}^{2}}{4} \\
& a_{7}=-\frac{r_{1}^{2}}{4} \\
& b_{1}=-X_{2 m}-x_{2} \\
& b_{4}=x_{2}-X_{2 m} \\
& b_{6}=\frac{1}{4}\left(x_{2}^{2}+X_{2 m}^{2}-r_{2}^{2}+2 X_{2 m} x_{2}\right) \\
& b_{7}=\frac{1}{4}\left(x_{2}^{2}+X_{2 m}^{2}-r_{2}^{2}-2 X_{2 m} x_{2}\right) \\
& c_{1}=-X_{3 m}-x_{3} \\
& c_{2}=-Y_{3 m}-y_{3} \\
& c_{3}=-Y_{3 m}+y_{3} \\
& c_{4}=x_{3}-X_{3 m} \\
& c_{5}=-Y_{3 m} x_{3}+X_{3 m} y_{3} \\
& c_{6}=\frac{1}{4}\left[x_{3}^{2}+y_{3}^{2}+X_{3 m}^{2}+Y_{3 m}^{2}-r_{3}^{2}-2\left(-X_{3 m} x_{3}-Y_{3 m} y_{3}\right)\right] \\
& c_{7}=\frac{1}{4}\left[x_{3}^{2}+y_{3}^{2}+X_{3 m}^{2}+Y_{3 m}^{2}-r_{3}^{2}+2\left(-X_{3 m} x_{3}-Y_{3 m} y_{3}\right)\right] .
\end{aligned}
$$


The three hyperboloids' equations are reduced by subtracting Eq.(5.10) from Eq.(5.11) and Eq.(5.12) to eliminate $X_{1}^{2}+X_{2}^{2}$ giving:

$$
\begin{aligned}
& X_{1}^{2}+X_{2}^{2}+a_{6} X_{3}^{2}+a_{7}=0 \\
& b_{1} X_{1} X_{3}+b_{4} X_{2}+e_{6} X_{3}^{2}+e_{7}=0 \\
& c_{1} X_{1} X_{3}+c_{2} X_{2} X_{3}+c_{3} X_{1}+c_{4} X_{2}+c_{5} X_{3}+e_{8} X_{3}^{2}+e_{9}=0 .
\end{aligned}
$$

Then eliminate $X_{3}^{2}$ from Eq.(5.15) by subtracting Eq.(5.14) and Eq.(5.15) from each other to produce:

$$
\begin{array}{r}
X_{1}^{2}+X_{2}^{2}+a_{6} X_{3}^{2}+a_{7}=0 \\
b_{1} X_{1} X_{3}+b_{4} X_{2}+e_{6} X_{3}^{2}+e_{7}=0 \\
f_{1} X_{1} X_{3}+f_{2} X_{2} X_{3}+f_{3} X_{1}+f_{5} X_{3}+f_{7}=0
\end{array}
$$

wherein:

$$
\begin{aligned}
& e_{6}=b_{6}-a_{6} \\
& e_{7}=b_{7}-a_{7} \\
& e_{8}=c_{6}-a_{6} \\
& e_{9}=c_{7}-a_{7} \\
& f_{1}=c_{1} e_{6}-b_{1} e_{8} \\
& f_{2}=c_{2} e_{6} \\
& f_{3}=c_{3} e_{6} \\
& f_{4}=c_{4} e_{6}-b_{4} e_{8} \\
& f_{5}=c_{5} e_{6} \\
& f_{7}=e_{6} e_{9}-e_{7} e_{8} .
\end{aligned}
$$

At this stage, a Gröbner basis is implemented for Eq.(5.16) by using the elimination order i.e lex order to eliminate variables to give the univariate polynomial for the forward kinematic problem with sixth order in the variable $X_{3}$. This determines the postures of the platform in this case. Doing the calculation by the method of Gröbner bases in Maple we obtain the univariate polynomial:

$$
A_{6} X_{3}^{6}+A_{5} X_{3}^{5}+A_{4} X_{3}^{4}+A_{3} X_{3}^{3}+A_{2} X_{3}^{2}+A_{1} X_{3}+A_{0}
$$


wherein:

$$
\begin{aligned}
A_{6}= & e_{6}^{2}\left(f_{1}^{2}+f_{2}^{2}\right)+a_{6} b_{1}^{2} f_{2}^{2} \\
A_{5}= & 2\left(a_{6} b_{1} f_{2}\left(b_{1} f_{4}-b_{4} f_{1}\right)-b_{1} e_{6} f_{1} f_{5}+e_{6}^{2}\left(f_{1} f_{3}+f_{2} f_{4}\right)\right) \\
A_{4}= & a_{6}\left(b_{1}^{2} f_{4}^{2}+b_{4}^{2} f_{1}^{2}\right)+b_{1}^{2}\left(a_{7} f_{2}^{2}+f_{5}^{2}\right)+e_{6}^{2}\left(f_{3}^{2}+f_{4}^{2}\right) \\
& -2 a_{6} b_{1} b_{4}\left(f_{1} f_{4}+f_{2} f_{3}\right)+e_{6}\left[b_{1}\left(f_{1} f_{7}+f_{3} f_{5}\right)+b_{4} f_{2} f_{5}-e_{7}\left(f_{1}^{2}+f_{2}^{2}\right)\right] \\
A_{3}= & 2 b_{4}\left[a_{6} f_{3}\left(b_{4} f_{1}-b_{1} f_{4}\right)-e_{6}\left(f_{2} f_{7}+f_{4} f_{5}\right)\right]-b_{1}\left[f_{1}\left(e_{7} f_{5}+a_{7} b_{4} f_{2}\right)\right. \\
& \left.+e_{6} f_{3} f_{7}-b_{1}\left(f_{5} f_{7}+a_{7} f_{4} f_{2}\right)\right]+2 e_{6} e_{7}\left(f_{1} f_{3}+f_{2} f_{4}\right) \\
A_{2}= & b_{4}^{2}\left(a_{7} f_{1}^{2}+f_{5}^{2}+a_{6} f_{3}^{2}\right)+b_{1}^{2}\left(f_{7}^{2}+a_{7} f_{4}^{2}\right)+e_{7}^{2}\left(f_{1}^{2}+f_{2}^{2}\right) \\
& +2 e_{6} e_{7}\left(f_{3}^{2}+f_{4}^{2}\right)-b_{4}\left[a_{7} b_{1}\left(f_{1} f_{4}+f_{2} f_{3}\right)+\left(e_{6} f_{4} f_{7}+e_{7} f_{2} f_{5}\right)\right] \\
& -b_{1} e_{7}\left(f_{1} f_{7}+f_{3} f_{5}\right) \\
A_{1}= & 2 e_{7}^{2}\left(f_{1} f_{3}+f_{2} f_{4}\right)-b_{1} f_{3}\left(e_{7} f_{7}+a_{7} b_{4} f_{4}\right)+b_{4}\left[b _ { 4 } \left(f_{5} f_{7}\right.\right. \\
& \left.\left.+a_{7} f_{1} f_{3}\right)-e_{7}\left(f_{2} f_{7}+f_{4} f_{5}\right)\right] \\
A_{0}= & \left(e_{7} f_{4}-b_{4} f_{7}\right)^{2}+f_{3}^{2}\left(e_{7}^{2}+a_{7} b_{4}^{2}\right) .
\end{aligned}
$$

Accordingly, there are six values for the variable $X_{3}$ evaluated by solving Eq.(5.17) numerically. Then once these values are available we obtain unique values of $X_{1}$ and $X_{2}$ by back substitution for each of the six values of $X_{3}$ [18]. The coefficients $A_{i}(i=0, \ldots, 6)$ are dependent on the leg lengths which are represented by the radii $r_{i}(i=1,2,3)$. This may allow us to examine how the number of real solutions may vary in terms of the leg lengths. This will be done by numerical examples.

Example 5.3.1 Solving Eq.(5.17) numerically by choosing numerical values for the point coordinates in Table 5.3 and putting the coefficients in terms of the leg lengths $r_{i}(i=1,2,3)$ and choose them to be $r_{1}=2, r_{2}=3, r_{3}=1$. Then, the numerical values for the circle's centers and the attachment points are listed in Table 5.4. 


\begin{tabular}{|c|c|c|c|}
\hline The leg & Circle center & Attachment point & Leg length \\
\hline leg 1 & $(0,0,1)$ & $(0,0,1)$ & $r_{1}=2$ \\
\hline leg 2 & $(2,0,1)$ & $(1,0,1)$ & $r_{2}=3$ \\
\hline leg 3 & $(1, \sqrt{3}, 1)$ & $\left(\frac{1}{2}, \frac{\sqrt{3}}{2}, 1\right)$ & $r_{3}=1$ \\
\hline
\end{tabular}

Table 5.4: numerical values for point coordinates

The six distinct roots of $X_{3}$ are then obtained and they are:

$$
\begin{aligned}
& \left(X_{3}\right)_{1}=-0.552 \\
& \left(X_{3}\right)_{2}=-0.356 \\
& \left(X_{3}\right)_{3}=-0.103-0.983 i \\
& \left(X_{3}\right)_{4}=-0.103+0.983 i \\
& \left(X_{3}\right)_{5}=0.482 \\
& \left(X_{3}\right)_{6}=6.405
\end{aligned}
$$

Now we determine the values of $X_{1}$ and $X_{2}$ by back substitution. This is done by substitute the four real values of $X_{3}$ into the last two equations of the system (5.16). Then when $X_{3}=\left(X_{3}\right)_{1}$ :

$$
X_{1}=0.868, X_{2}=0.742 \text {. }
$$

Similarly, we determine the value of $X_{1}$ and $X_{2}$ for the other values of $X_{3}$. When $X_{3}=\left(X_{3}\right)_{2}$ :

$$
X_{1}=1.036, X_{2}=0.233 \text {. }
$$

When $X_{3}=\left(X_{3}\right)_{5}$, the value of $X_{1}$ and $X_{2}$ are:

$$
X_{1}=-0.939, X_{2}=0.590
$$

When $X_{3}=\left(X_{3}\right)_{6}$, we get:

$$
X_{1}=1.758, X_{2}=6.239 \text {. }
$$

Now we have four real sets of variables $X_{1}, X_{2}, X_{3}$ that are specified from specific inputs which allow us to determine the characteristic parameters $(a, b, \phi)$ the preimages of each set by substituting in Eq.(3.13). They are listed in Table 5.5. So, 


\begin{tabular}{|c|c|c|c|}
\hline Solution of $X_{3}$ & $a$ & $b$ & $\phi$ (deg.) \\
\hline$\left(X_{3}\right)_{1}$ & 0.403 & -1.958 & -57.79 \\
\hline$\left(X_{3}\right)_{2}$ & -0.241 & -1.986 & -39.19 \\
\hline$\left(X_{3}\right)_{5}$ & 0.223 & 1.985 & 51.47 \\
\hline$\left(X_{3}\right)_{6}$ & 0.833 & 1.818 & 162.3 \\
\hline
\end{tabular}

Table 5.5: The four real platform solutions

there are four real postures of the platform for the given leg lengths and its assembly modes which are illustrated in Fig. 5.6. If we vary the leg lengths in example 5.3.1 and choose $r_{1}=\frac{1}{2}, r_{2}=\frac{3}{2}, r_{3}=\frac{1}{4}$, the univariate polynomial in $X_{3}$ has only two real roots. They are $\left(X_{3}\right)_{1}=-5.506,\left(X_{3}\right)_{2}=-4.068$. If we vary them again and choose $r_{1}=0, r_{2}=0, r_{3}=0$, the univariate polynomial in $X_{3}$ has no real solutions. According to [6], varying the leg lengths shows that the number of real solutions for C-C-C class legs can be 4, 2 or 0 . However, it has not been possible to find a set of leg lengths giving 6 real solutions for this particular example, although, the maximum number of real solutions for the C-C-C class leg is six as shown in [6] by Gosselin's example.

\subsubsection{L-L-L Class Legs}

This planar manipulator has three legs of PR-Type denoted $L$. To calculate its univariate polynomial we first determine the constraint surface equations for each leg, given by equations (5.7), (5.9), applying the upper sign.

Example 5.3.2 These equations are obtained by substituting the base points and the inputs joint from Table(5.6) where the joint inputs are angles and they are selected for convenience. 

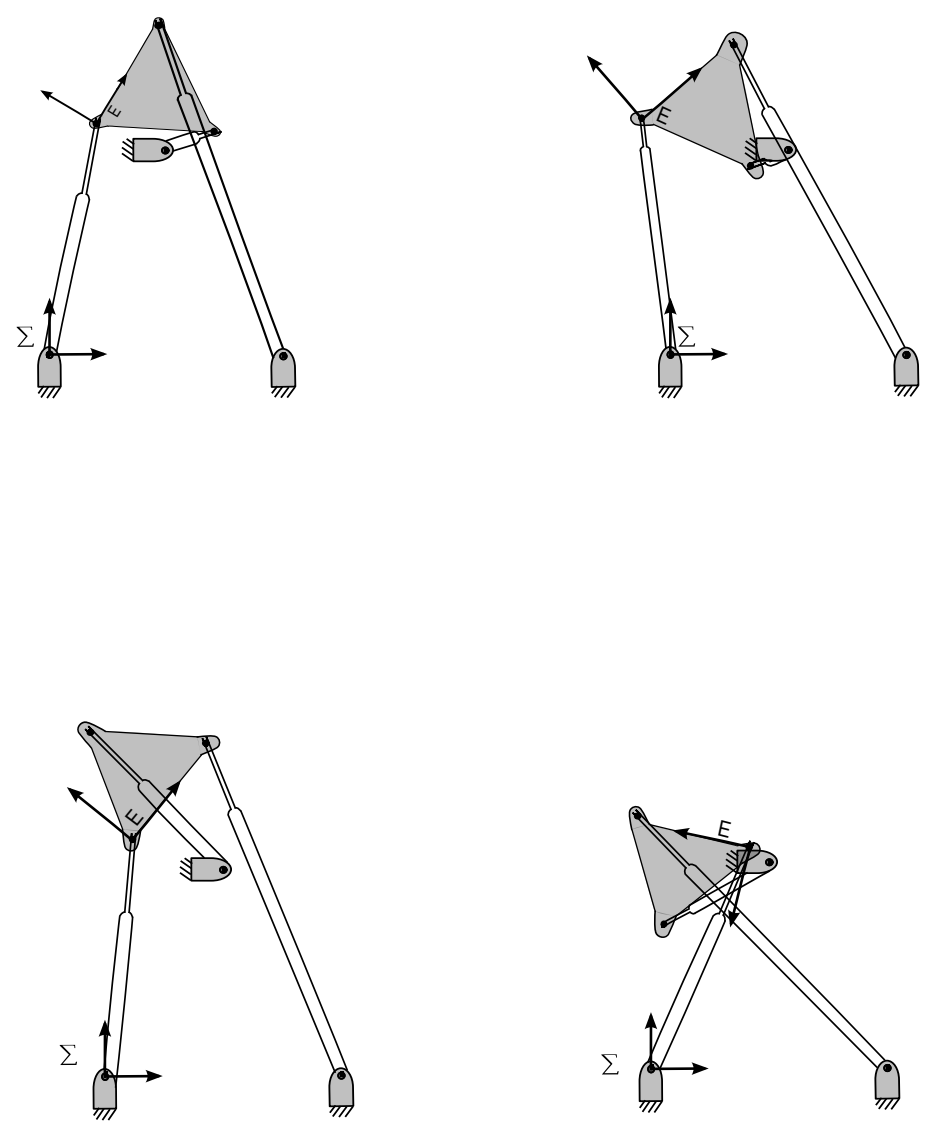

Figure 5.6: The four postures for RPR platform

The constraint equations for the three legs are:

$$
\begin{aligned}
& -\frac{1}{4} \sqrt{3} X_{1} X_{3}+\frac{1}{4} X_{2} X_{3}-\frac{1}{4} X_{1}-\frac{1}{4} \sqrt{3} X_{2}=0 \\
& \sqrt{2} X_{3}^{2}-\frac{1}{4} \sqrt{2} X_{1} X_{3}+\frac{1}{4} \sqrt{2} X_{2} X_{3}-\frac{1}{4} \sqrt{2} X_{1}-\frac{1}{4} \sqrt{2} X_{2}+\frac{1}{2} \sqrt{2} X_{3}+\frac{1}{2} \sqrt{2}=0 \\
& 2 X_{3}^{2}-\frac{1}{2} X_{2} X_{3}+\frac{1}{2} X_{1}-\frac{1}{2} X_{3}+1=0
\end{aligned}
$$

Using Gröbner bases, the univariate polynomial in $X_{3}$ is:

$$
44 X_{3}^{2}+(3 \sqrt{3}+7) X_{3}+22=0
$$

So the univariate polynomial is of degree 2 , unlike the first type. The roots of this polynomial are $\left(X_{3}\right)_{1}=-\frac{3}{88} \sqrt{3}-\frac{7}{88}-\frac{1}{88} i \sqrt{3796-42 \sqrt{3}},\left(X_{3}\right)_{2}=-\frac{3}{88} \sqrt{3}-$ 


\begin{tabular}{|c|c|c|c|c|}
\hline Leg & Input angles & $\left(X_{A}: Y_{A}: Z_{A}\right)$ & $(x: y: z)$ & $\left(k_{1}: k_{2}: k_{3}\right)$ \\
\hline leg 1 & $\frac{\pi}{3}$ & $(0: 0: 1)$ & $(0: 0: 1)$ & $\left(-\frac{\sqrt{3}}{2}: \frac{1}{4}: 0\right)$ \\
\hline $\operatorname{leg} 2$ & $\frac{\pi}{4}$ & $(6: 0: 1)$ & $(2: 0: 1)$ & $\left(-\frac{\sqrt{2}}{4}: \frac{\sqrt{2}}{4}: 3 \sqrt{2}\right)$ \\
\hline $\operatorname{leg} 3$ & $\pi$ & $(3: 6: 1)$ & $(1: 2: 1)$ & $\left(-\frac{1}{2}: 0: 3\right)$ \\
\hline
\end{tabular}

Table 5.6: Point coordinates

$\frac{7}{88}+\frac{1}{88} i \sqrt{3796-42 \sqrt{3}}$. This means that the platform of these particular design parameters stay in its zero position since the roots of $X_{3}$ are complex. The following investigation shows that the degree of the univariate polynomial describe the maximum number of real solutions. This investigation follows as in [9].

The calculation of the univariate polynomial in a mechanism of class C-C-C accords with what has been found by Gosselin [6], Merlet [13] and Husty [10], among others, that there are at most six real solutions for the forward kinematic problem. Viewing this problem in algebraic geometry, three quadric surfaces in the image space intersect typically in eight solutions by Bezout's Theorem. Then there are two missing points of intersections in the solution set of the univariate polynomial. We ask naturally where are they? The constraint surface equation for a leg of RR-Type is seen to be hyperboloid of one sheet, and it has special structure since it is formed by a family of circles. The circles are the cross-section $X_{3}=$ constant . The planes $X_{3}=$ constant intersect all the hyperboloids in circles where the complex points lie on all circles. We generate three hyberboloids by the first factor of Eq.(5.4) and intersect them with the plane $X_{4}=0$ to demonstrate this. We set in this factor $X_{4}=0, z=1$ and $k_{0}=1$. Then, we obtain the following three hyperboloids $H_{1}, H_{2}$ and $H_{3}$ after we set $k_{1}=k_{2}=x=y=0$ in $H_{1}, K_{2}=Y=0$ in $H_{2}$, and $k_{1}, k_{2}, x$, and $y$ in $H_{3}$ are corresponded to non-zero values. The three hyperboloids are:

$$
\begin{gathered}
H_{1}: X_{1}^{2}+X_{2}^{2}+\frac{1}{4} k_{3} X_{3}^{2}=0, \\
H_{2}: X_{1}^{2}+X_{2}^{2}+\frac{1}{4}\left(x^{2}+k_{3}-2 k_{1}\right) X_{3}^{2}+\left(k_{1}-x\right) X_{1} X_{3}-k_{2} X_{1} X_{4}+k_{2} X_{2} X_{3}+\left(x+k_{1}\right) X_{2} X_{4}=0,
\end{gathered}
$$


$H_{3}: X_{1}^{2}+X_{2}^{2}+\frac{1}{4}\left[x^{2}+y^{2}+k_{3}-2\left(k_{1} x+k_{2} y\right)\right] X_{3}^{2}+\left(k_{1}-x\right) X_{1} X_{3}+\left(k_{2}-y\right) X_{2} X_{3}=0$.

The imaginary conjugate points $J_{1}(1: i: 0: 0)$ and $J_{2}(1:-i: 0: 0)$ are the intersection points of the three constraint hyperboloids for the three legs and the plane $X_{4}=0$. Note that $J_{1}$ and $J_{2}$ do not have pre-images as they do not correspond to a real displacements. By Bezout's Theorem, the three constraint hyperboloids intersect in eight points. The two complex points $J_{1}$ and $J_{2}$ are considered as two of the intersection points and they are common to every circular hyperboloid. The line of intersection between the planes $X_{3}=0$ and $X_{4}=0$ passes through these imaginary conjugate points $J_{1}(1: i: 0: 0)$ and $J_{2}(1:-i: 0: 0)$ where the hyperboloids intersect. Thus, $J_{1}$ and $J_{2}$ are the missing points, and hence there are at most six real solutions or assembly models for any given set of leg lengths.

However, when the constraint surface equation is a hyperbolic paraboloid, corresponding to the legs of $P R$-Type or $R P$-Type, the constraint is linear, not circular. So, we want to determine in how many points these surfaces can intersect with the plane $X_{4}=0$. Substituting $X_{4}=0$ in the first factor of Eq.(5.4) and then $x=y=0$ in the first leg equation, $x=b_{1}, y=0$ in the second leg equation and $x=c_{1}, y=c_{2}$ in the third leg equation, we obtain:

$$
\begin{gathered}
H P_{1}: \frac{1}{4} k_{3} X_{3}^{2}+\left(k_{1}\right) X_{1} X_{3}+\left(k_{2}\right) X_{2} X_{3}=0 \\
H P_{2}: \frac{1}{4}\left[k_{3}-2 k_{1} b_{1}\right] X_{3}^{2}+\left(k_{1}\right) X_{1} X_{3}+\left(k_{2}\right) X_{2} X_{3}=0 \\
H P_{3}: \frac{1}{4}\left[k_{3}-2\left(k_{1} c_{1}+k_{2} c_{2}\right)\right] X_{3}^{2}+\left(k_{1}\right) X_{1} X_{3}+\left(k_{2}\right) X_{2} X_{3}=0 .
\end{gathered}
$$

Clearly, this set of equations is always satisfied by the image point coordinate:

$$
\left(X_{1}: X_{2}: X_{3}: X_{4}\right)=\left(X_{1}: X_{2}: 0: 0\right)
$$

This means that the line of intersection of the planes $X_{3}=0, X_{4}=0$ are contained in such a set. By Bezout's Theorem, two constraint hyperbolic paraboloids intersect in a curve of fourth degree. The common intersection points of every line in each 


\begin{tabular}{|c|c|c|c|c|}
\hline Leg & The joint inputs & $\left(x_{A}: y_{A}: z_{A}\right)$ & $(X: Y: Z)$ & $\left(k_{1}: k_{2}: k_{3}\right)$ \\
\hline leg 1 & $\frac{\pi}{2}$ & $(0: 0: 1)$ & $(0: 0: 1)$ & $\left(-\frac{1}{2}: 0: 0\right)$ \\
\hline leg 2 & $\pi$ & $(3: 0: 1)$ & $(2: 0: 1)$ & $\left(0:-\frac{1}{2}: 0\right)$ \\
\hline leg 3 & $\frac{\pi}{3}$ & $(1: 3: 1)$ & $(1: 2: 1)$ & $\left(-\frac{\sqrt{3}}{4}: \frac{1}{4}: \frac{\sqrt{3}-3}{2}\right)$ \\
\hline
\end{tabular}

Table 5.7: Point coordinates

regulus of both surfaces form a twisted cubic. Then, the intersection curve of both surfaces decomposes into two curves one of which is the line of intersection of the planes $X_{3}=X_{4}=0$ and the other is the twisted cubic. The twisted cubic must intersect the line $X_{3}=X_{4}=0$ in two points since this line is contained in the set of constraint hyperbolic paraboloids. As we have three constraint equations, there are three twisted cubic curves which intersect with the line $X_{3}=X_{4}=0$. The intersection points of the three surfaces are determined by the points of intersection of the three twisted cubics with the line. Then the three surfaces intersect in six points that lie on the line $X_{3}=X_{4}=0$. Hence, these points do not correspond to real displacements and then the poses they represent are not physically valid. There are two additional points according to Bezout's Theorem. Therefore, for a leg of $R P$-Type or $P R$-Type there are at most two real solutions and this is consistent with the solution for the forward kinematic problem for these types of legs which give a univariate polynomial of degree two.

\subsubsection{T-T-T Class Legs}

A planar manipulator with three legs of $R P$-Type (where $\mathrm{T}$ - is assigned to this type of leg as shown) has at most two assembly modes in the image space since it is the inversion of $P R$-Type. This will be validated by the following example by deriving its univariate polynomial. The constraint surface equation for each leg is given by Eq.(5.8) and Eq.(5.9), where the lower sign applies. Again we select the joint inputs for convenience. 


\begin{tabular}{|c|c|c|c|}
\hline Solution of $X_{3}$ & $a$ & $b$ & $\phi($ deg. $)$ \\
\hline$\left(X_{3}\right)_{1}$ & 0.723 & -0.961 & -143.03 \\
\hline$\left(X_{3}\right)_{2}$ & 0.364 & 0.753 & -24.6 \\
\hline
\end{tabular}

Table 5.8: The two real platform solutions

Example 5.3.3 By using the platform's geometry and joint inputs in Table 5.7.

The constraint equations for the three legs are:

$$
\begin{aligned}
& -\frac{1}{2} X_{1} X_{3}+\frac{1}{2} X_{2}=0 \\
& -\frac{1}{2} X_{2} X_{3}-\frac{1}{2} X_{1}+X_{3}=0 \\
& \frac{2 \sqrt{3}-5}{8} X_{3}^{2}-\frac{\sqrt{3}}{4} X_{1} X_{3}+\frac{1}{4} X_{2} X_{3}+\frac{1}{4} X_{1}+\frac{\sqrt{3}}{4} X_{2}-\frac{2 \sqrt{3}+1}{4} X_{3}-\frac{1}{8}=0
\end{aligned}
$$

Using the Gröbner bases method; the univariate polynomial in $X_{3}$ is:

$$
13 X_{3}^{2}+(16 \sqrt{3}+14) X_{3}+2 \sqrt{3}+5=0
$$

which has degree two. The roots of this polynomial are $\left(X_{3}\right)_{1}=-2.991,\left(X_{3}\right)_{2}=$ -0.2177 . In this case both of the solutions are real. Now we determine the values of $X_{1}$ and $X_{2}$ by back substitution. Then, when $X_{3}=\left(X_{3}\right)_{1}$ :

$$
X_{1}=-0.601, X_{2}=1.799
$$

When $X_{3}=\left(X_{3}\right)_{2}$ :

$$
X_{1}=-0.416, X_{2}=0.1
$$

The two assembly poses are now determined by the parameters $(a, b, \phi)$ by substitute the two real sets of variables $X_{1}, X_{2}, X_{3}$ in Eq.(3.13). They are listed in Table 5.8 . 


\subsection{Conclusion}

Using kinematic mapping to solve the difficult problem in kinematics, the forward kinematic problem, is valuable. We attempted to solve this problem for the symmetric platforms, namely the univariate polynomials by the method of Gröbner bases. The univariate polynomials for platforms involving circular constraint are of degree six while for platforms involving linear constraint are of degree two. The degrees of these univariate polynomials describe the maximum number of real solutions. The solutions are explained in terms of algebraic geometry, by using Bezout's Theorem. The calculations of univariate polynomials could be extended for example by considering varying joint inputs and design parameters. 


\section{Appendix A}

The derivation of the univariate polynomial for a leg of RR-Type by Maple: 
with (Groebner);

[Basis, FGLM, HilbertDimension, HilbertPolynomial, HilbertSeries, Homogenize, InitialForm,

InterReduce, IsBasis, Is Proper, IsZeroDimensional, LeadingCoefficient, LeadingMonomial, LeadingTerm, MatrixOrder, MaximalIndependentSet, MonomialOrder, MultiplicationMatrix, MultivariateCyclicVector, NormalForm, NormalSet, RationalUnivariateRepresentation, Reduce, RememberBasis, SPolynomial, Solve, SuggestVariableOrder, Support, TestOrder, ToricIdealBasis, TrailingTerm, UnivariatePolynomial, Walk, WeightedDegree ]

$$
\begin{aligned}
& F:=\left[X[1]^{2}+X[2]^{2}+a[6] \cdot X[3]^{2}+a[7], b[1] \cdot X[1] \cdot X[3]+b[4] \cdot X[2]+e[6] \cdot X[3]^{2}+e[7],\right. \\
& f[1] \cdot X[1] \cdot X[3]+f[2] \cdot X[2] \cdot X[3]+f[3] \cdot X[1]+f[5] \cdot X[3]+f[7]] \\
& {\left[X_{1}^{2}+X_{2}^{2}+a_{6} X_{3}^{2}+a_{7}, b_{1} X_{1} X_{3}+b_{4} X_{2}+e_{6} X_{3}^{2}+e_{7}, f_{1} X_{1} X_{3}+f_{2} X_{2} X_{3}+f_{3} X_{1}+f_{5} X_{3}\right.} \\
& \left.+f_{7}\right] \\
& G:=\operatorname{Basis}(F, \operatorname{plex}(X[1], X[2], X[3])) \\
& {\left[b_{4}^{2} f_{7}^{2}+e_{7}^{2} f_{3}^{2}+a_{7} b_{4}^{2} f_{3}^{2}+\left(-2 b_{1} e_{6} f_{3} f_{7}-2 b_{1} e_{7} f_{1} f_{5}+2 b_{1}^{2} f_{5} f_{7}+4 e_{6} e_{7} f_{1} f_{3}\right.\right.} \\
& \left.-2 b_{4} e_{6} f_{2} f_{7}+2 a_{6} b_{4}^{2} f_{1} f_{3}-2 a_{7} b_{1} b_{4} f_{1} f_{2}\right) X_{3}^{3}+\left(-2 b_{4} e_{7} f_{2} f_{7}-2 b_{1} e_{7} f_{3} f_{7}+2\right. \\
& \left.e_{7}^{2} f_{1} f_{3}+2 a_{7} b_{4}^{2} f_{1} f_{3}+2 b_{4}^{2} f_{5} f_{7}\right) X_{3}+\left(-2 b_{1} e_{7} f_{1} f_{7}-2 b_{1} e_{7} f_{3} f_{5}+e_{7}^{2} f_{1}^{2}+b_{1}^{2} f_{7}^{2}+b_{4}^{2}\right. \\
& \left.f_{5}^{2}+a_{7} b_{4}^{2} f_{1}^{2}-2 b_{4} e_{7} f_{2} f_{5}-2 a_{7} b_{1} b_{4} f_{2} f_{3}+a_{6} b_{4}^{2} f_{3}^{2}+2 e_{6} e_{7} f_{3}^{2}+e_{7}^{2} f_{2}^{2}\right) X_{3}^{2}+\left(b_{1}^{2} f_{5}^{2}\right. \\
& -2 b_{1} e_{6} f_{3} f_{5}-2 a_{6} b_{1} b_{4} f_{2} f_{3}-2 b_{4} e_{6} f_{2} f_{5}-2 b_{1} e_{6} f_{1} f_{7}+2 e_{6} e_{7} f_{1}^{2}+a_{7} b_{1}^{2} f_{2}^{2} \\
& \left.+2 e_{6} e_{7} f_{2}^{2}+e_{6}^{2} f_{3}^{2}+a_{6} b_{4}^{2} f_{1}^{2}\right) X_{3}^{4}+\left(-2 b_{1} e_{6} f_{1} f_{5}+2 e_{6}^{2} f_{1} f_{3}-2 a_{6} b_{1} b_{4} f_{1} f_{2}\right) X_{3}^{5} \\
& +\left(f_{2}^{2} a_{6} b_{1}^{2}+e_{6}^{2} f_{2}^{2}+e_{6}^{2} f_{1}^{2}\right) X_{3}^{6},\left(2 b_{1}^{4} b_{4} e_{6} f_{1}^{2} f_{2} f_{3} f_{5} f_{7}-2 b_{1}^{3} b_{4} e_{6} e_{7} f_{1}^{3} f_{2} f_{3} f_{5}-2\right. \\
& b_{1}^{3} b_{4} e_{6} e_{7} f_{1} f_{2}^{3} f_{3} f_{5}-a_{6} b_{1}^{4} b_{4}^{2} f_{1}^{2} f_{2}^{2} f_{3} f_{7}-a_{6} b_{1}^{3} b_{4}^{3} f_{1}^{3} f_{2} f_{3} f_{5}-a_{6} b_{1}^{3} b_{4}^{3} f_{1} f_{2}^{3} f_{3} f_{5}-2 b_{1}^{2} \\
& b_{4}^{2} e_{6}^{2} f_{1} f_{2}^{2} f_{3}^{2} f_{5}+4 b_{1}^{2} b_{4} e_{6}^{2} e_{7} f_{1}^{3} f_{2} f_{3}^{2}+2 b_{1}^{2} b_{4} e_{6}^{2} e_{7} f_{1} f_{2}^{3} f_{3}^{2}-b_{1} b_{4}^{3} e_{6}^{2} f_{1}^{3} f_{2} f_{3} f_{5}-b_{1} b_{4}^{3} \\
& e_{6}^{2} f_{1} f_{2}^{3} f_{3} f_{5}+3 b_{1} b_{4}^{2} e_{6}^{2} e_{7} f_{1}^{3} f_{2}^{2} f_{3}+b_{1} b_{4}^{2} e_{6}^{2} e_{7} f_{1} f_{2}^{4} f_{3}+2 b_{1}^{4} b_{4} e_{6} f_{1} f_{2} f_{3}^{2} f_{5}^{2}+4 b_{1}^{4} b_{4} e_{6} \\
& f_{2}^{3} f_{3} f_{5} f_{7}-4 b_{1}^{4} e_{6} e_{7} f_{1}^{2} f_{2}^{2} f_{3} f_{7}+2 b_{1}^{3} b_{4}^{2} e_{6} f_{1}^{2} f_{2}^{2} f_{5} f_{7}+b_{1}^{3} b_{4}^{2} e_{6} f_{1} f_{2}^{2} f_{3} f_{5}^{2}-5 b_{1}^{3} b_{4} e_{6}^{2} \\
& f_{1}^{2} f_{2} f_{3}^{2} f_{7}-b_{1}^{3} b_{4} e_{6}^{2} f_{1} f_{2} f_{3}^{3} f_{5}-2 b_{1}^{3} b_{4} e_{6} e_{7} f_{1}^{2} f_{2}^{3} f_{7}-5 b_{1}^{2} b_{4}^{2} e_{6}^{2} f_{1}^{2} f_{2}^{2} f_{3} f_{7}-a_{6} b_{1}^{3} \\
& b_{4}^{2} e_{6} f_{1} f_{2}^{2} f_{3}^{3}+a_{6} b_{1}^{3} b_{4}^{2} e_{7} f_{1}^{3} f_{2}^{2} f_{3}+a_{6} b_{1}^{3} b_{4}^{2} e_{7} f_{1} f_{2}^{4} f_{3}+2 a_{6} b_{1}^{2} b_{4}^{3} e_{6} f_{1}^{3} f_{2} f_{3}^{2}+a_{6} b_{1} \\
& b_{4}^{4} e_{6} f_{1}^{3} f_{2}^{2} f_{3}-a_{7} b_{1}^{5} b_{4} f_{1} f_{2}^{3} f_{3} f_{5}+2 a_{7} b_{1}^{4} b_{4} e_{6} f_{1} f_{2}^{3} f_{3}^{2}+a_{7} b_{1}^{3} b_{4}^{2} e_{6} f_{1}^{3} f_{2}^{2} f_{3}+a_{7} b_{1}^{3} \\
& b_{4}^{2} e_{6} f_{1} f_{2}^{4} f_{3}+a_{6} b_{1}^{5} b_{4} f_{2}^{3} f_{3}^{2} f_{7}+a_{6} b_{1}^{4} b_{4}^{2} f_{2}^{4} f_{3} f_{7}+2 b_{1}^{3} e_{6} e_{7}^{2} f_{1}^{3} f_{2}^{2} f_{3}+2 b_{1}^{3} e_{6} e_{7}^{2} f_{1} f_{2}^{4} f_{3} \\
& -2 b_{1}^{2} b_{4}^{2} e_{6}^{2} f_{1}^{4} f_{3} f_{7}-b_{1}^{2} b_{4}^{2} e_{6}^{2} f_{2}^{4} f_{3} f_{7}-b_{1} b_{4}^{3} e_{6}^{2} f_{1}^{4} f_{2} f_{7}-b_{1} b_{4}^{3} e_{6}^{2} f_{1}^{2} f_{2}^{3} f_{7}+2 b_{1} b_{4}^{2} e_{6}^{2} e_{7} \\
& f_{1}^{5} f_{3}+a_{6} b_{1} b_{4}^{4} e_{6} f_{1}^{5} f_{3}+a_{7} b_{1}^{5} e_{7} f_{1} f_{2}^{4} f_{3}-b_{1}^{5} b_{4} f_{1} f_{2} f_{3} f_{5}^{3}+2 b_{1}^{5} e_{6} f_{1} f_{2}^{2} f_{3} f_{7}^{2}+2 b_{1}^{5} e_{6} \\
& f_{2}^{2} f_{3}^{2} f_{5} f_{7}+b_{1}^{5} e_{7} f_{1} f_{2}^{2} f_{3} f_{5}^{2}+2 b_{1}^{4} b_{4} e_{6} f_{1} f_{2}^{3} f_{7}^{2}-2 b_{1}^{4} e_{6} e_{7} f_{2}^{4} f_{3} f_{7}+b_{1}^{3} b_{4}^{2} e_{6} f_{1}^{3} f_{3} f_{5}^{2}+2
\end{aligned}
$$


$b_{1}^{3} b_{4}^{2} e_{6} f_{2}^{4} f_{5} f_{7}-2 b_{1}^{3} b_{4} e_{6}^{2} f_{2}^{3} f_{3}^{2} f_{7}-2 b_{1}^{3} b_{4} e_{6} e_{7} f_{2}^{5} f_{7}-b_{1}^{3} e_{6}^{2} e_{7} f_{1} f_{2}^{2} f_{3}^{3}-a_{7} b_{1}^{6} f_{2}^{4} f_{3} f_{7}$ $\left.-a_{7} b_{1}^{5} b_{4} f_{2}^{5} f_{7}-b_{1}^{6} f_{2}^{2} f_{3} f_{5}^{2} f_{7}-b_{1}^{5} b_{4} f_{2}^{3} f_{5}^{2} f_{7}-b_{1}^{4} e_{6}^{2} f_{2}^{2} f_{3}^{3} f_{7}\right) X_{3}^{3}+\left(2 b_{1}^{4} b_{4} e_{7}\right.$ $f_{1}^{2} f_{2} f_{3} f_{5} f_{7}-5 b_{1}^{3} b_{4} e_{6} e_{7} f_{1}^{2} f_{2} f_{3}^{2} f_{7}+2 b_{1}^{2} b_{4}^{3} e_{6} f_{1}^{2} f_{2} f_{3} f_{5} f_{7}-4 b_{1}^{2} b_{4}^{2} e_{6} e_{7} f_{1}^{2} f_{2}^{2} f_{3} f_{7}$ $-a_{7} b_{1}^{4} b_{4}^{2} f_{1}^{2} f_{2}^{2} f_{3} f_{7}-a_{7} b_{1}^{3} b_{4}^{3} f_{1}^{3} f_{2} f_{3} f_{5}+2 b_{1}^{4} b_{4} e_{7} f_{2}^{3} f_{3} f_{5} f_{7}-b_{1}^{3} b_{4}^{2} e_{6} f_{1}^{2} f_{3}^{2} f_{5} f_{7}+3$ $b_{1}^{3} b_{4}^{2} e_{6} f_{1} f_{2}^{2} f_{3} f_{7}^{2}-2 b_{1}^{3} b_{4}^{2} e_{6} f_{2}^{2} f_{3}^{2} f_{5} f_{7}+2 b_{1}^{3} b_{4}^{2} e_{7} f_{1}^{2} f_{2}^{2} f_{5} f_{7}-b_{1}^{3} b_{4} e_{7}^{2} f_{1}^{3} f_{2} f_{3} f_{5}-$ $b_{1}^{3} b_{4} e_{7}^{2} f_{1} f_{2}^{3} f_{3} f_{5}-2 b_{1}^{2} b_{4}^{2} e_{6} e_{7} f_{1}^{4} f_{3} f_{7}+2 b_{1}^{2} b_{4} e_{6} e_{7}^{2} f_{1}^{3} f_{2} f_{3}^{2}+b_{1} b_{4}^{4} e_{6} f_{1}^{2} f_{2}^{2} f_{5} f_{7}+b_{1}$ $b_{4}^{3} e_{6}^{2} f_{1}^{2} f_{2} f_{3}^{2} f_{7}-b_{1} b_{4}^{3} e_{6} e_{7} f_{1}^{4} f_{2} f_{7}-b_{1} b_{4}^{3} e_{6} e_{7} f_{1}^{2} f_{2}^{3} f_{7}+b_{1} b_{4}^{2} e_{6} e_{7}^{2} f_{1}^{3} f_{2}^{2} f_{3}-a_{7} b_{1}^{3}$ $b_{4}^{3} f_{1} f_{2}^{3} f_{3} f_{5}-a_{7} b_{1}^{3} b_{4}^{2} e_{6} f_{1} f_{2}^{2} f_{3}^{3}+a_{7} b_{1}^{3} b_{4}^{2} e_{7} f_{1}^{3} f_{2}^{2} f_{3}+a_{7} b_{1}^{3} b_{4}^{2} e_{7} f_{1} f_{2}^{4} f_{3}+2 a_{7} b_{1}^{2} b_{4}^{3} e_{6}$ $f_{1}^{3} f_{2} f_{3}^{2}+a_{7} b_{1} b_{4}^{4} e_{6} f_{1}^{3} f_{2}^{2} f_{3}-b_{1}^{5} b_{4} f_{1} f_{2} f_{3} f_{5} f_{7}^{2}+3 b_{1}^{4} b_{4} e_{6} f_{1} f_{2} f_{3}^{2} f_{7}^{2}-2 b_{1}^{4} b_{4} e_{6} f_{2}$ $f_{3}^{3} f_{5} f_{7}+a_{7} b_{1}^{5} b_{4} f_{2}^{3} f_{3}^{2} f_{7}+a_{7} b_{1}^{4} b_{4}^{2} f_{2}^{4} f_{3} f_{7}+a_{7} b_{1} b_{4}^{4} e_{6} f_{1}^{5} f_{3}+b_{1}^{5} b_{4} f_{2} f_{3}^{2} f_{5}^{2} f_{7}+3$ $b_{1}^{5} e_{7} f_{1} f_{2}^{2} f_{3} f_{7}^{2}-2 b_{1}^{4} b_{4}^{2} f_{1} f_{2}^{2} f_{5} f_{7}^{2}+2 b_{1}^{4} b_{4} e_{7} f_{1} f_{2}^{3} f_{7}^{2}-3 b_{1}^{4} e_{7}^{2} f_{1}^{2} f_{2}^{2} f_{3} f_{7}-b_{1}^{3} b_{4}^{3} f_{1}^{2} f_{2}$ $f_{5}^{2} f_{7}+b_{1}^{3} b_{4}^{2} e_{6} f_{1}^{3} f_{3} f_{7}^{2}+2 b_{1}^{3} b_{4}^{2} e_{7} f_{2}^{4} f_{5} f_{7}+b_{1}^{3} b_{4} e_{6}^{2} f_{2} f_{3}^{4} f_{7}-b_{1}^{3} b_{4} e_{7}^{2} f_{1}^{2} f_{2}^{3} f_{7}-b_{1}^{3} e_{6}$ $e_{7}^{2} f_{1} f_{2}^{2} f_{3}^{3}+b_{1}^{2} b_{4}^{3} e_{6} f_{1}^{3} f_{2} f_{7}^{2}+b_{1}^{2} b_{4}^{3} e_{6} f_{1} f_{2}^{3} f_{7}^{2}+2 b_{1}^{2} b_{4}^{2} e_{6}^{2} f_{2}^{2} f_{3}^{3} f_{7}+b_{1} b_{4}^{4} e_{6} f_{1}^{4} f_{5} f_{7}+b_{1}$ $b_{4}^{3} e_{6}^{2} f_{2}^{3} f_{3}^{2} f_{7}+b_{1} b_{4}^{2} e_{6} e_{7}^{2} f_{1}^{5} f_{3}-b_{1}^{4} e_{7}^{2} f_{2}^{4} f_{3} f_{7}-b_{1}^{3} b_{4}^{3} f_{2}^{3} f_{5}^{2} f_{7}-b_{1}^{3} b_{4} e_{7}^{2} f_{2}^{5} f_{7}+b_{1}^{3} e_{7}^{3} f_{1}^{3}$ $\left.f_{2}^{2} f_{3}+b_{1}^{3} e_{7}^{3} f_{1} f_{2}^{4} f_{3}-b_{1}^{6} f_{2}^{2} f_{3} f_{7}^{3}-b_{1}^{5} b_{4} f_{2}^{3} f_{7}^{3}\right) X_{3}+\left(6 b_{1}^{4} b_{4} e_{6} f_{1} f_{2} f_{3}^{2} f_{5} f_{7}+6 b_{1}^{3}\right.$ $b_{4}^{2} e_{6} f_{1} f_{2}^{2} f_{3} f_{5} f_{7}-6 b_{1}^{3} b_{4} e_{6} e_{7} f_{1}^{2} f_{2} f_{3}^{2} f_{5}-4 b_{1}^{3} b_{4} e_{6} e_{7} f_{1} f_{2}^{3} f_{3} f_{7}+2 b_{1}^{2} b_{4}^{2} e_{6} e_{7} f_{1}^{2}$ $f_{2}^{2} f_{3} f_{5}-a_{6} b_{1}^{4} b_{4}^{2} f_{1} f_{2}^{2} f_{3}^{2} f_{7}-a_{6} b_{1}^{3} b_{4}^{3} f_{1}^{2} f_{2} f_{3}^{2} f_{5}+a_{6} b_{1}^{3} b_{4}^{2} e_{7} f_{1}^{2} f_{2}^{2} f_{3}^{2}+3 a_{6} b_{1}^{2} b_{4}^{3} e_{6} f_{1}^{2} f_{2}$ $f_{3}^{3}+a_{6} b_{1} b_{4}^{4} e_{6} f_{1}^{2} f_{2}^{2} f_{3}^{2}-2 b_{1}^{2} b_{4} e_{6} e_{7}^{2} f_{1}^{2} f_{2}^{3} f_{3}-6 b_{1} b_{4}^{3} e_{6}^{2} f_{1}^{3} f_{2} f_{3} f_{7}+5 b_{1} b_{4}^{3} e_{6}^{2} f_{1}^{2} f_{2} f_{3}^{2} f_{5}$ $-4 b_{1} b_{4}^{3} e_{6}^{2} f_{1} f_{2}^{3} f_{3} f_{7}-3 b_{1} b_{4}^{2} e_{6}^{2} e_{7} f_{1}^{2} f_{2}^{2} f_{3}^{2}-2 b_{1}^{2} b_{4}^{2} e_{6} e_{7} f_{1}^{3} f_{2}^{2} f_{7}-2 b_{1}^{2} b_{4}^{2} e_{6} e_{7} f_{1} f_{2}^{4} f_{7}$ $+4 b_{1}^{2} b_{4}^{2} e_{6} e_{7} f_{2}^{4} f_{3} f_{5}+5 b_{1}^{2} b_{4} e_{6}^{2} e_{7} f_{1}^{2} f_{2} f_{3}^{3}+2 a_{7} b_{1}^{5} b_{4} f_{1} f_{2}^{3} f_{3} f_{7}+2 a_{7} b_{1}^{4} b_{4}^{2} f_{1}^{2} f_{2}^{2} f_{3} f_{5}$ $-2 a_{7} b_{1}^{4} b_{4} e_{7} f_{1}^{2} f_{2}^{3} f_{3}-5 a_{7} b_{1}^{3} b_{4}^{2} e_{6} f_{1}^{2} f_{2}^{2} f_{3}^{2}-2 a_{7} b_{1}^{2} b_{4}^{3} e_{6} f_{1}^{4} f_{2} f_{3}-2 a_{7} b_{1}^{2} b_{4}^{3} e_{6} f_{1}^{2}$ $f_{2}^{3} f_{3}-2 b_{1}^{5} b_{4} f_{1} f_{2} f_{3} f_{5}^{2} f_{7}+4 b_{1}^{5} e_{7} f_{1} f_{2}^{2} f_{3} f_{5} f_{7}+2 b_{1}^{4} b_{4} e_{7} f_{1}^{2} f_{2} f_{3} f_{5}^{2}+2 b_{1}^{4} b_{4} e_{7} f_{1}$ $f_{2}^{3} f_{5} f_{7}-4 b_{1}^{4} e_{6} e_{7} f_{1} f_{2}^{2} f_{3}^{2} f_{7}+2 b_{1}^{3} b_{4}^{2} e_{6} f_{1}^{3} f_{3} f_{5} f_{7}-4 b_{1}^{3} b_{4} e_{6}^{2} f_{1} f_{2} f_{3}^{3} f_{7}+6 b_{1}^{3} b_{4} e_{6} e_{7}$ $f_{2}^{3} f_{3}^{2} f_{5}+2 b_{1}^{2} b_{4}^{3} e_{6} f_{1}^{3} f_{2} f_{5} f_{7}-2 b_{1}^{2} b_{4}^{3} e_{6} f_{1}^{2} f_{2} f_{3} f_{5}^{2}+2 b_{1}^{2} b_{4}^{3} e_{6} f_{1} f_{2}^{3} f_{5} f_{7}-8 b_{1}^{2} b_{4}^{2} e_{6}^{2} f_{1}$ $f_{2}^{2} f_{3}^{2} f_{7}-2 b_{1}^{2} b_{4}^{2} e_{6} e_{7} f_{1}^{4} f_{3} f_{5}-a_{6} b_{1}^{4} b_{4}^{2} f_{2}^{2} f_{3}^{3} f_{5}+a_{6} b_{1}^{4} b_{4} e_{7} f_{2}^{3} f_{3}^{3}-a_{6} b_{1}^{3} b_{4}^{3} f_{2}^{3} f_{3}^{2} f_{5}$ $+a_{6} b_{1}^{3} b_{4}^{2} e_{6} f_{2}^{2} f_{3}^{4}+a_{6} b_{1}^{3} b_{4}^{2} e_{7} f_{2}^{4} f_{3}^{2}+a_{6} b_{1}^{2} b_{4}^{3} e_{6} f_{2}^{3} f_{3}^{3}+a_{6} b_{1} b_{4}^{4} e_{6} f_{1}^{4} f_{3}^{2}+a_{7} b_{1}^{5} b_{4} f_{2}^{3}$ $f_{3}^{2} f_{5}+a_{7} b_{1}^{4} b_{4}^{2} f_{1} f_{2}^{4} f_{7}+a_{7} b_{1}^{4} b_{4}^{2} f_{2}^{4} f_{3} f_{5}-a_{7} b_{1}^{4} b_{4} e_{6} f_{2}^{3} f_{3}^{3}-a_{7} b_{1}^{4} b_{4} e_{7} f_{2}^{5} f_{3}-a_{7} b_{1}^{3}$ 
$b_{4}^{2} e_{6} f_{2}^{4} f_{3}^{2}-b_{1}^{4} b_{4}^{2} f_{1} f_{2}^{2} f_{5}^{2} f_{7}+2 b_{1}^{3} e_{6} e_{7}^{2} f_{1}^{2} f_{2}^{2} f_{3}^{2}-2 b_{1}^{2} b_{4}^{3} e_{6} f_{2}^{3} f_{3} f_{5}^{2}-b_{1}^{2} b_{4}^{2} e_{6}^{2} f_{1}^{3} f_{3}^{2} f_{7}+$ $b_{1}^{2} b_{4}^{2} e_{6}^{2} f_{1}^{2} f_{3}^{3} f_{5}+6 b_{1}^{2} b_{4}^{2} e_{6}^{2} f_{2}^{2} f_{3}^{3} f_{5}-4 b_{1}^{2} b_{4} e_{6}^{2} e_{7} f_{2}^{3} f_{3}^{3}-2 b_{1}^{2} b_{4} e_{6} e_{7}^{2} f_{2}^{5} f_{3}+3 b_{1} b_{4}^{3} e_{6}^{2}$ $f_{2}^{3} f_{3}^{2} f_{5}+2 b_{1} b_{4}^{2} e_{6}^{2} e_{7} f_{1}^{4} f_{3}^{2}-3 b_{1} b_{4}^{2} e_{6}^{2} e_{7} f_{2}^{4} f_{3}^{2}+b_{4}^{4} e_{6}^{2} f_{1}^{2} f_{2}^{2} f_{3} f_{5}-b_{4}^{3} e_{6}^{2} e_{7} f_{1}^{4} f_{2} f_{3}-b_{4}^{3}$ $e_{6}^{2} e_{7} f_{1}^{2} f_{2}^{3} f_{3}+4 b_{1}^{4} b_{4} e_{6} f_{2}^{3} f_{3} f_{7}^{2}-3 b_{1}^{4} b_{4} e_{6} f_{2} f_{3}^{3} f_{5}^{2}-b_{1}^{4} b_{4} e_{7} f_{2}^{3} f_{3} f_{5}^{2}+2 b_{1}^{4} e_{6} e_{7} f_{2}^{2} f_{3}^{3} f_{5}$ $-2 b_{1}^{4} e_{7}^{2} f_{1}^{2} f_{2}^{2} f_{3} f_{5}+2 b_{1}^{3} b_{4}^{2} e_{6} f_{1}^{2} f_{2}^{2} f_{7}^{2}-b_{1}^{3} b_{4}^{2} e_{6} f_{1}^{2} f_{3}^{2} f_{5}^{2}-5 b_{1}^{3} b_{4}^{2} e_{6} f_{2}^{2} f_{3}^{2} f_{5}^{2}+3 b_{1}^{3} b_{4}$ $e_{6}^{2} f_{2} f_{3}^{4} f_{5}-b_{1}^{2} b_{4} e_{6}^{3} f_{2} f_{3}^{5}-2 b_{1} b_{4}^{2} e_{6}^{3} f_{2}^{2} f_{3}^{4}+b_{4}^{4} e_{6}^{2} f_{1}^{4} f_{3} f_{5}-b_{4}^{4} e_{6}^{2} f_{1}^{3} f_{2}^{2} f_{7}-b_{4}^{3} e_{6}^{3} f_{1}^{2} f_{2} f_{3}^{3}$ $-a_{7} b_{1}^{5} e_{7} f_{2}^{4} f_{3}^{2}-2 b_{1}^{6} f_{2}^{2} f_{3} f_{5} f_{7}^{2}-2 b_{1}^{5} b_{4} f_{2}^{3} f_{5} f_{7}^{2}+b_{1}^{5} b_{4} f_{2} f_{3}^{2} f_{5}^{3}+2 b_{1}^{5} e_{6} f_{2}^{2} f_{3}^{2} f_{7}^{2}-$ $b_{1}^{5} e_{7} f_{2}^{2} f_{3}^{2} f_{5}^{2}+b_{1}^{4} b_{4}^{2} f_{2}^{2} f_{3} f_{5}^{3}+2 b_{1}^{3} b_{4}^{2} e_{6} f_{2}^{4} f_{7}^{2}-b_{1}^{3} e_{6}^{2} e_{7} f_{2}^{2} f_{3}^{4}-2 b_{1}^{3} e_{6} e_{7}^{2} f_{2}^{4} f_{3}^{2}-b_{4}^{3} e_{6}^{3}$ $\left.f_{2}^{3} f_{3}^{3}-b_{4}^{4} e_{6}^{2} f_{1}^{5} f_{7}\right) X_{3}^{2}+\left(2 b_{1}^{4} b_{4} e_{7} f_{2}^{2} f_{3}^{3} f_{5}+2 b_{1}^{3} b_{4}^{2} e_{6} f_{2} f_{3}^{4} f_{5}+4 b_{1}^{3} b_{4}^{2} e_{7} f_{2}^{3} f_{3}^{2} f_{5}-2\right.$ $b_{1}^{3} b_{4} e_{6} e_{7} f_{2}^{2} f_{3}^{4}+b_{1}^{2} b_{4}^{4} f_{1}^{3} f_{2} f_{5} f_{7}-b_{1}^{2} b_{4}^{4} f_{1}^{2} f_{2} f_{3} f_{5}^{2}+b_{1}^{2} b_{4}^{4} f_{1} f_{2}^{3} f_{5} f_{7}-b_{1}^{2} b_{4}^{3} e_{6} f_{1}^{3} f_{3}^{2} f_{7}+$ $b_{1}^{2} b_{4}^{3} e_{6} f_{1}^{2} f_{3}^{3} f_{5}+4 b_{1}^{2} b_{4}^{3} e_{6} f_{2}^{2} f_{3}^{3} f_{5}-b_{1}^{2} b_{4}^{3} e_{7} f_{1}^{3} f_{2}^{2} f_{7}-b_{1}^{2} b_{4}^{3} e_{7} f_{1} f_{2}^{4} f_{7}+2 b_{1}^{2} b_{4}^{3} e_{7}$ $f_{2}^{4} f_{3} f_{5}-4 b_{1}^{2} b_{4}^{2} e_{6} e_{7} f_{2}^{3} f_{3}^{3}-b_{1}^{2} b_{4}^{2} e_{7}^{2} f_{1}^{2} f_{2}^{3} f_{3}+2 b_{1} b_{4}^{4} e_{6} f_{2}^{3} f_{3}^{2} f_{5}-2 b_{1} b_{4}^{3} e_{6} e_{7} f_{2}^{4} f_{3}^{2}+$ $b_{4}^{5} e_{6} f_{1}^{2} f_{2}^{2} f_{3} f_{5}-b_{4}^{4} e_{6} e_{7} f_{1}^{4} f_{2} f_{3}-b_{4}^{4} e_{6} e_{7} f_{1}^{2} f_{2}^{3} f_{3}+b_{1}^{4} b_{4}^{2} f_{1} f_{2} f_{3}^{2} f_{5} f_{7}-b_{1}^{4} b_{4} e_{7} f_{1} f_{2}^{2} f_{3}^{2} f_{7}$ $+2 b_{1}^{3} b_{4}^{3} f_{1} f_{2}^{2} f_{3} f_{5} f_{7}-3 b_{1}^{3} b_{4}^{2} e_{6} f_{1} f_{2} f_{3}^{3} f_{7}-2 b_{1}^{3} b_{4}^{2} e_{7} f_{1} f_{2}^{3} f_{3} f_{7}-6 b_{1}^{2} b_{4}^{3} e_{6} f_{1} f_{2}^{2} f_{3}^{2} f_{7}$ $+2 b_{1}^{2} b_{4}^{3} e_{7} f_{1}^{2} f_{2}^{2} f_{3} f_{5}-b_{1}^{2} b_{4}^{2} e_{6} e_{7} f_{1}^{2} f_{2} f_{3}^{3}-5 b_{1} b_{4}^{4} e_{6} f_{1}^{3} f_{2} f_{3} f_{7}+4 b_{1} b_{4}^{4} e_{6} f_{1}^{2} f_{2} f_{3}^{2} f_{5}$ $-3 b_{1} b_{4}^{4} e_{6} f_{1} f_{2}^{3} f_{3} f_{7}-4 b_{1} b_{4}^{3} e_{6} e_{7} f_{1}^{2} f_{2}^{2} f_{3}^{2}+b_{1}^{5} b_{4} f_{2}^{2} f_{3}^{2} f_{7}^{2}+2 b_{1}^{4} b_{4}^{2} f_{2}^{3} f_{3} f_{7}^{2}-b_{1}^{4} b_{4}^{2} f_{2}$ $f_{3}^{3} f_{5}^{2}+b_{1}^{3} b_{4}^{3} f_{1}^{2} f_{2}^{2} f_{7}^{2}-2 b_{1}^{3} b_{4}^{3} f_{2}^{2} f_{3}^{2} f_{5}^{2}-2 b_{1}^{3} b_{4} e_{7}^{2} f_{2}^{4} f_{3}^{2}-b_{1}^{2} b_{4}^{4} f_{2}^{3} f_{3} f_{5}^{2}-b_{1}^{2} b_{4}^{2} e_{6}^{2} f_{2} f_{3}^{5}$ $-b_{1}^{2} b_{4}^{2} e_{7}^{2} f_{2}^{5} f_{3}-2 b_{1} b_{4}^{3} e_{6}^{2} f_{2}^{2} f_{3}^{4}+b_{4}^{5} e_{6} f_{1}^{4} f_{3} f_{5}-b_{4}^{5} e_{6} f_{1}^{3} f_{2}^{2} f_{7}-b_{4}^{4} e_{6}^{2} f_{1}^{2} f_{2} f_{3}^{3}-b_{1}^{4} e_{7}^{2}$ $\left.f_{2}^{3} f_{3}^{3}+b_{1}^{3} b_{4}^{3} f_{2}^{4} f_{7}^{2}-b_{4}^{5} e_{6} f_{1}^{5} f_{7}-b_{4}^{4} e_{6}^{2} f_{2}^{3} f_{3}^{3}\right) X_{2}+\left(2 a_{6} b_{1}^{5} b_{4} f_{1} f_{2}^{3} f_{3} f_{7}+2 a_{6} b_{1}^{4} b_{4}^{2} f_{1}^{2}\right.$ $f_{2}^{2} f_{3} f_{5}-2 a_{6} b_{1}^{4} b_{4} e_{7} f_{1}^{2} f_{2}^{3} f_{3}-5 a_{6} b_{1}^{3} b_{4}^{2} e_{6} f_{1}^{2} f_{2}^{2} f_{3}^{2}-2 a_{6} b_{1}^{2} b_{4}^{3} e_{6} f_{1}^{4} f_{2} f_{3}-2 a_{6} b_{1}^{2}$ $b_{4}^{3} e_{6} f_{1}^{2} f_{2}^{3} f_{3}+2 b_{1}^{5} e_{6} f_{1} f_{2}^{2} f_{3} f_{5} f_{7}+2 b_{1}^{4} b_{4} e_{6} f_{1}^{2} f_{2} f_{3} f_{5}^{2}+2 b_{1}^{4} b_{4} e_{6} f_{1} f_{2}^{3} f_{5} f_{7}-2$ $b_{1}^{4} e_{6} e_{7} f_{1}^{2} f_{2}^{2} f_{3} f_{5}-5 b_{1}^{3} b_{4} e_{6}^{2} f_{1}^{2} f_{2} f_{3}^{2} f_{5}-2 b_{1}^{3} b_{4} e_{6}^{2} f_{1} f_{2}^{3} f_{3} f_{7}-b_{1}^{2} b_{4}^{2} e_{6}^{2} f_{1}^{2} f_{2}^{2} f_{3} f_{5}-$ $b_{1}^{2} b_{4} e_{6}^{2} e_{7} f_{1}^{2} f_{2}^{3} f_{3}+b_{1}^{3} e_{6}^{2} e_{7} f_{1}^{2} f_{2}^{2} f_{3}^{2}-2 b_{1}^{2} b_{4}^{2} e_{6}^{2} f_{1}^{4} f_{3} f_{5}-b_{1}^{2} b_{4}^{2} e_{6}^{2} f_{1}^{3} f_{2}^{2} f_{7}-b_{1}^{2} b_{4}^{2} e_{6}^{2} f_{1}$ $f_{2}^{4} f_{7}+b_{1}^{2} b_{4}^{2} e_{6}^{2} f_{2}^{4} f_{3} f_{5}+3 b_{1}^{2} b_{4} e_{6}^{3} f_{1}^{2} f_{2} f_{3}^{3}-b_{1}^{2} b_{4} e_{6}^{2} e_{7} f_{2}^{5} f_{3}+a_{6} b_{1}^{5} b_{4} f_{2}^{3} f_{3}^{2} f_{5}+a_{6} b_{1}^{4}$ $b_{4}^{2} f_{1} f_{2}^{4} f_{7}+a_{6} b_{1}^{4} b_{4}^{2} f_{2}^{4} f_{3} f_{5}-a_{6} b_{1}^{4} b_{4} e_{6} f_{2}^{3} f_{3}^{3}-a_{6} b_{1}^{4} b_{4} e_{7} f_{2}^{5} f_{3}-a_{6} b_{1}^{3} b_{4}^{2} e_{6} f_{2}^{4} f_{3}^{2}-2$ $b_{1}^{4} e_{6}^{2} f_{1} f_{2}^{2} f_{3}^{2} f_{7}+b_{1}^{3} b_{4} e_{6}^{2} f_{2}^{3} f_{3}^{2} f_{5}-a_{6} b_{1}^{5} e_{7} f_{2}^{4} f_{3}^{2}-b_{1}^{3} e_{6}^{2} e_{7} f_{2}^{4} f_{3}^{2}-b_{1}^{2} b_{4} e_{6}^{3} f_{2}^{3} f_{3}^{3}+b_{1} b_{4}^{2}$ $\left.e_{6}^{3} f_{1}^{4} f_{3}^{2}-b_{1} b_{4}^{2} e_{6}^{3} f_{2}^{4} f_{3}^{2}\right) X_{3}^{4}+\left(-b_{1}^{3} b_{4} e_{6}^{2} f_{2}^{5} f_{7}+b_{1} b_{4}^{2} e_{6}^{3} f_{1}^{5} f_{3}+a_{6} b_{1}^{5} e_{7} f_{1} f_{2}^{4} f_{3}-b_{1}^{4} e_{6}^{2}\right.$ 
$f_{1}^{2} f_{2}^{2} f_{3} f_{7}+b_{1}^{3} e_{6}^{2} e_{7} f_{1} f_{2}^{4} f_{3}+2 b_{1}^{2} b_{4} e_{6}^{3} f_{1}^{3} f_{2} f_{3}^{2}-b_{1}^{3} b_{4} e_{6}^{2} f_{1}^{2} f_{2}^{3} f_{7}+b_{1}^{3} e_{6}^{2} e_{7} f_{1}^{3} f_{2}^{2} f_{3}-a_{6}$ $b_{1}^{6} f_{2}^{4} f_{3} f_{7}-a_{6} b_{1}^{5} b_{4} f_{2}^{5} f_{7}+2 b_{1}^{2} b_{4} e_{6}^{3} f_{1} f_{2}^{3} f_{3}^{2}+2 b_{1} b_{4}^{2} e_{6}^{3} f_{1}^{3} f_{2}^{2} f_{3}+2 a_{6} b_{1}^{4} b_{4} e_{6} f_{1} f_{2}^{3} f_{3}^{2}$ $+a_{6} b_{1}^{3} b_{4}^{2} e_{6} f_{1}^{3} f_{2}^{2} f_{3}-b_{1}^{4} e_{6}^{2} f_{2}^{4} f_{3} f_{7}-a_{6} b_{1}^{5} b_{4} f_{1} f_{2}^{3} f_{3} f_{5}-b_{1}^{3} b_{4} e_{6}^{2} f_{1} f_{2}^{3} f_{3} f_{5}+b_{1} b_{4}^{2} e_{6}^{3} f_{1}$ $\left.f_{2}^{4} f_{3}+a_{6} b_{1}^{3} b_{4}^{2} e_{6} f_{1} f_{2}^{4} f_{3}-b_{1}^{3} b_{4} e_{6}^{2} f_{1}^{3} f_{2} f_{3} f_{5}\right) X_{3}^{5}-a_{7} b_{1}^{4} b_{4}^{2} f_{1} f_{2}^{2} f_{3}^{2} f_{7}-a_{7} b_{1}^{3} b_{4}^{3} f_{1}^{2} f_{2}$ $f_{3}^{2} f_{5}+a_{7} b_{1}^{3} b_{4}^{2} e_{7} f_{1}^{2} f_{2}^{2} f_{3}^{2}+3 a_{7} b_{1}^{2} b_{4}^{3} e_{6} f_{1}^{2} f_{2} f_{3}^{3}+a_{7} b_{1} b_{4}^{4} e_{6} f_{1}^{2} f_{2}^{2} f_{3}^{2}+2 b_{1}^{2} b_{4}^{2} e_{7}^{2} f_{1}^{2}$ $f_{2}^{2} f_{3} f_{5}+2 b_{1}^{2} b_{4} e_{6} e_{7}^{2} f_{1}^{2} f_{2} f_{3}^{3}+2 b_{1} b_{4}^{3} e_{6} e_{7} f_{2}^{3} f_{3}^{2} f_{5}-3 b_{1} b_{4}^{2} e_{6} e_{7}^{2} f_{1}^{2} f_{2}^{2} f_{3}^{2}+b_{4}^{4} e_{6} e_{7} f_{1}^{2}$ $f_{2}^{2} f_{3} f_{5}+2 b_{1}^{3} b_{4} e_{6} e_{7} f_{2} f_{3}^{4} f_{5}-b_{1}^{3} b_{4} e_{7}^{2} f_{1}^{2} f_{2} f_{3}^{2} f_{5}-2 b_{1}^{3} b_{4} e_{7}^{2} f_{1} f_{2}^{3} f_{3} f_{7}+3 b_{1}^{2} b_{4}^{3} e_{6}$ $f_{1}^{2} f_{2} f_{3} f_{7}^{2}+b_{1}^{2} b_{4}^{3} e_{7} f_{1}^{3} f_{2} f_{5} f_{7}-b_{1}^{2} b_{4}^{3} e_{7} f_{1}^{2} f_{2} f_{3} f_{5}^{2}+b_{1}^{2} b_{4}^{3} e_{7} f_{1} f_{2}^{3} f_{5} f_{7}-b_{1}^{2} b_{4}^{2} e_{6} e_{7} f_{1}^{3}$ $f_{3}^{2} f_{7}+b_{1}^{2} b_{4}^{2} e_{6} e_{7} f_{1}^{2} f_{3}^{3} f_{5}+4 b_{1}^{2} b_{4}^{2} e_{6} e_{7} f_{2}^{2} f_{3}^{3} f_{5}-3 b_{1} b_{4}^{3} e_{6} e_{7} f_{1} f_{2}^{3} f_{3} f_{7}+b_{1}^{4} b_{4} e_{7} f_{1} f_{2}$ $f_{3}^{2} f_{5} f_{7}+2 b_{1}^{3} b_{4}^{2} e_{7} f_{1} f_{2}^{2} f_{3} f_{5} f_{7}-3 b_{1}^{3} b_{4} e_{6} e_{7} f_{1} f_{2} f_{3}^{3} f_{7}-6 b_{1}^{2} b_{4}^{2} e_{6} e_{7} f_{1} f_{2}^{2} f_{3}^{2} f_{7}-5 b_{1}$ $b_{4}^{3} e_{6} e_{7} f_{1}^{3} f_{2} f_{3} f_{7}+4 b_{1} b_{4}^{3} e_{6} e_{7} f_{1}^{2} f_{2} f_{3}^{2} f_{5}-a_{7} b_{1}^{4} b_{4}^{2} f_{2}^{2} f_{3}^{3} f_{5}+a_{7} b_{1}^{4} b_{4} e_{7} f_{2}^{3} f_{3}^{3}-a_{7} b_{1}^{3}$ $b_{4}^{3} f_{2}^{3} f_{3}^{2} f_{5}+a_{7} b_{1}^{3} b_{4}^{2} e_{6} f_{2}^{2} f_{3}^{4}+a_{7} b_{1}^{3} b_{4}^{2} e_{7} f_{2}^{4} f_{3}^{2}+a_{7} b_{1}^{2} b_{4}^{3} e_{6} f_{2}^{3} f_{3}^{3}+a_{7} b_{1} b_{4}^{4} e_{6} f_{1}^{4} f_{3}^{2}+3$ $b_{1}^{3} b_{4} e_{7}^{2} f_{2}^{3} f_{3}^{2} f_{5}+b_{1}^{2} b_{4}^{3} e_{6} f_{2}^{3} f_{3} f_{7}^{2}-b_{1}^{2} b_{4}^{3} e_{7} f_{2}^{3} f_{3} f_{5}^{2}-b_{1}^{2} b_{4}^{2} e_{7}^{2} f_{1}^{3} f_{2}^{2} f_{7}-b_{1}^{2} b_{4}^{2} e_{7}^{2} f_{1} f_{2}^{4} f_{7}$ $+2 b_{1}^{2} b_{4}^{2} e_{7}^{2} f_{2}^{4} f_{3} f_{5}-b_{1}^{2} b_{4} e_{6}^{2} e_{7} f_{2} f_{3}^{5}-3 b_{1}^{2} b_{4} e_{6} e_{7}^{2} f_{2}^{3} f_{3}^{3}-b_{1}^{2} b_{4} e_{7}^{3} f_{1}^{2} f_{2}^{3} f_{3}+b_{1} b_{4}^{4} e_{6}$ $f_{1}^{2} f_{2}^{2} f_{7}^{2}-2 b_{1} b_{4}^{2} e_{6}^{2} e_{7} f_{2}^{2} f_{3}^{4}+b_{1} b_{4}^{2} e_{6} e_{7}^{2} f_{1}^{4} f_{3}^{2}-2 b_{1} b_{4}^{2} e_{6} e_{7}^{2} f_{2}^{4} f_{3}^{2}+b_{4}^{4} e_{6} e_{7} f_{1}^{4} f_{3} f_{5}-$ $b_{4}^{4} e_{6} e_{7} f_{1}^{3} f_{2}^{2} f_{7}-b_{4}^{3} e_{6}^{2} e_{7} f_{1}^{2} f_{2} f_{3}^{3}-b_{4}^{3} e_{6} e_{7}^{2} f_{1}^{4} f_{2} f_{3}-b_{4}^{3} e_{6} e_{7}^{2} f_{1}^{2} f_{2}^{3} f_{3}-b_{1}^{4} b_{4}^{2} f_{2}^{2} f_{3} f_{5} f_{7}^{2}$ $+3 b_{1}^{4} b_{4} e_{7} f_{2}^{3} f_{3} f_{7}^{2}-b_{1}^{4} b_{4} e_{7} f_{2} f_{3}^{3} f_{5}^{2}-2 b_{1}^{4} e_{7}^{2} f_{1} f_{2}^{2} f_{3}^{2} f_{7}-b_{1}^{3} b_{4}^{3} f_{1}^{2} f_{2} f_{5} f_{7}^{2}+b_{1}^{3} b_{4}^{2} e_{6} f_{2}^{2}$ $f_{3}^{2} f_{7}^{2}+2 b_{1}^{3} b_{4}^{2} e_{7} f_{1}^{2} f_{2}^{2} f_{7}^{2}-2 b_{1}^{3} b_{4}^{2} e_{7} f_{2}^{2} f_{3}^{2} f_{5}^{2}+b_{1}^{5} e_{7} f_{2}^{2} f_{3}^{2} f_{7}^{2}-b_{1}^{4} b_{4}^{2} f_{1} f_{2}^{2} f_{7}^{3}+b_{1}^{4} e_{7}^{2} f_{2}^{2}$ $f_{3}^{3} f_{5}-b_{1}^{3} b_{4}^{3} f_{2}^{3} f_{5} f_{7}^{2}+2 b_{1}^{3} b_{4}^{2} e_{7} f_{2}^{4} f_{7}^{2}-b_{1}^{3} e_{6} e_{7}^{2} f_{2}^{2} f_{3}^{4}+b_{1}^{3} e_{7}^{3} f_{1}^{2} f_{2}^{2} f_{3}^{2}-b_{1}^{2} b_{4} e_{7}^{3} f_{2}^{5} f_{3}$ $+b_{1} b_{4}^{4} e_{6} f_{1}^{4} f_{7}^{2}-b_{4}^{4} e_{6} e_{7} f_{1}^{5} f_{7}-b_{4}^{3} e_{6}^{2} e_{7} f_{2}^{3} f_{3}^{3}-b_{1}^{3} e_{7}^{3} f_{2}^{4} f_{3}^{2},\left(2 b_{1}^{4} b_{4} e_{6} f_{1} f_{2}^{2} f_{3} f_{5} f_{7}-2\right.$ $b_{1}^{3} b_{4} e_{6} e_{7} f_{1}^{2} f_{2}^{2} f_{3} f_{5}+a_{6} b_{1}^{2} b_{4}^{3} e_{7} f_{1}^{4} f_{2}^{2}+a_{6} b_{1}^{2} b_{4}^{3} e_{7} f_{1}^{2} f_{2}^{4}-b_{1}^{5} b_{4} f_{1} f_{2}^{2} f_{5}^{2} f_{7}+2 b_{1}^{4} b_{4} e_{6}$ $f_{1}^{2} f_{2}^{2} f_{7}^{2}+3 b_{1}^{4} b_{4} e_{6} f_{2}^{2} f_{3}^{2} f_{5}^{2}+b_{1}^{4} b_{4} e_{7} f_{1}^{2} f_{2}^{2} f_{5}^{2}-2 b_{1}^{4} e_{6} e_{7} f_{2}^{3} f_{3}^{2} f_{5}+5 b_{1}^{3} b_{4}^{2} e_{6} f_{2}^{3} f_{3} f_{5}^{2}$ $-3 b_{1}^{3} b_{4} e_{6}^{2} f_{2}^{2} f_{3}^{3} f_{5}+2 b_{1}^{3} e_{6} e_{7}^{2} f_{1}^{2} f_{2}^{3} f_{3}+3 b_{1}^{2} b_{4}^{3} e_{6} f_{1}^{2} f_{2}^{2} f_{5}^{2}-6 b_{1}^{2} b_{4}^{2} e_{6}^{2} f_{2}^{3} f_{3}^{2} f_{5}-4 b_{1}^{2}$ $b_{4}^{2} e_{6} e_{7} f_{2}^{5} f_{5}+4 b_{1}^{2} b_{4} e_{6}^{2} e_{7} f_{2}^{4} f_{3}^{2}+2 b_{1}^{2} b_{4} e_{6} e_{7}^{2} f_{1}^{4} f_{2}^{2}+4 b_{1}^{2} b_{4} e_{6} e_{7}^{2} f_{1}^{2} f_{2}^{4}-b_{1} b_{4}^{3} e_{6}^{2} f_{1}^{3}$ $f_{2}^{2} f_{7}+b_{1} b_{4}^{3} e_{6}^{2} f_{1} f_{2}^{4} f_{7}-3 b_{1} b_{4}^{3} e_{6}^{2} f_{2}^{4} f_{3} f_{5}+3 b_{1} b_{4}^{2} e_{6}^{2} e_{7} f_{2}^{5} f_{3}+a_{6} b_{1}^{4} b_{4}^{2} f_{2}^{3} f_{3}^{2} f_{5}-a_{6}$ $b_{1}^{4} b_{4} e_{7} f_{2}^{4} f_{3}^{2}-a_{6} b_{1}^{3} b_{4}^{3} f_{1}^{3} f_{2}^{2} f_{7}-a_{6} b_{1}^{3} b_{4}^{3} f_{1} f_{2}^{4} f_{7}+a_{6} b_{1}^{3} b_{4}^{3} f_{2}^{4} f_{3} f_{5}-a_{6} b_{1}^{3} b_{4}^{2} e_{6} f_{2}^{3} f_{3}^{3}$ $-a_{6} b_{1}^{3} b_{4}^{2} e_{7} f_{2}^{5} f_{3}-a_{6} b_{1}^{2} b_{4}^{4} f_{1}^{4} f_{2} f_{5}-a_{6} b_{1}^{2} b_{4}^{4} f_{1}^{2} f_{2}^{3} f_{5}-a_{6} b_{1}^{2} b_{4}^{3} e_{6} f_{2}^{4} f_{3}^{2}-a_{6} b_{1}^{3} b_{4}^{3} f_{1}^{2}$ 
$f_{2}^{2} f_{3} f_{5}+a_{6} b_{1}^{3} b_{4}^{2} e_{7} f_{1}^{2} f_{2}^{3} f_{3}+3 a_{6} b_{1} b_{4}^{4} e_{6} f_{1}^{4} f_{2} f_{3}+a_{6} b_{1} b_{4}^{4} e_{6} f_{1}^{2} f_{2}^{3} f_{3}-2 b_{1}^{4} e_{6} e_{7} f_{1}$ $f_{2}^{3} f_{3} f_{7}+2 b_{1}^{3} b_{4}^{2} e_{6} f_{1}^{3} f_{2} f_{5} f_{7}+3 b_{1}^{3} b_{4}^{2} e_{6} f_{1}^{2} f_{2} f_{3} f_{5}^{2}+2 b_{1}^{3} b_{4}^{2} e_{6} f_{1} f_{2}^{3} f_{5} f_{7}-b_{1}^{3} b_{4} e_{6}^{2} f_{1} f_{2}^{2}$ $f_{3}^{2} f_{7}-4 b_{1}^{3} b_{4} e_{6} e_{7} f_{1}^{3} f_{2}^{2} f_{7}-4 b_{1}^{3} b_{4} e_{6} e_{7} f_{1} f_{2}^{4} f_{7}-6 b_{1}^{3} b_{4} e_{6} e_{7} f_{2}^{4} f_{3} f_{5}-6 b_{1}^{2} b_{4}^{2} e_{6}^{2}$ $f_{1}^{3} f_{2} f_{3} f_{7}-2 b_{1}^{2} b_{4}^{2} e_{6}^{2} f_{1}^{2} f_{2} f_{3}^{2} f_{5}-2 b_{1}^{2} b_{4}^{2} e_{6} e_{7} f_{1}^{4} f_{2} f_{5}-6 b_{1}^{2} b_{4}^{2} e_{6} e_{7} f_{1}^{2} f_{2}^{3} f_{5}+2 b_{1}^{2} b_{4}$ $e_{6}^{2} e_{7} f_{1}^{2} f_{2}^{2} f_{3}^{2}-7 b_{1} b_{4}^{3} e_{6}^{2} f_{1}^{2} f_{2}^{2} f_{3} f_{5}+6 b_{1} b_{4}^{2} e_{6}^{2} e_{7} f_{1}^{4} f_{2} f_{3}+9 b_{1} b_{4}^{2} e_{6}^{2} e_{7} f_{1}^{2} f_{2}^{3} f_{3}+3 a_{7} b_{1}^{3}$ $b_{4}^{2} e_{6} f_{1}^{2} f_{2}^{3} f_{3}-a_{7} b_{1}^{5} b_{4} f_{1} f_{2}^{4} f_{7}-a_{7} b_{1}^{5} b_{4} f_{2}^{4} f_{3} f_{5}-a_{7} b_{1}^{4} b_{4}^{2} f_{1}^{2} f_{2}^{3} f_{5}+a_{7} b_{1}^{4} b_{4} e_{6} f_{2}^{4} f_{3}^{2}$ $+a_{7} b_{1}^{4} b_{4} e_{7} f_{1}^{2} f_{2}^{4}+a_{7} b_{1}^{3} b_{4}^{2} e_{6} f_{2}^{5} f_{3}+a_{7} b_{1}^{2} b_{4}^{3} e_{6} f_{1}^{4} f_{2}^{2}+a_{7} b_{1}^{2} b_{4}^{3} e_{6} f_{1}^{2} f_{2}^{4}+a_{7} b_{1}^{5} e_{7}$ $f_{2}^{5} f_{3}-a_{7} b_{1}^{4} b_{4}^{2} f_{2}^{5} f_{5}+a_{7} b_{1}^{4} b_{4} e_{7} f_{2}^{6}-2 b_{4}^{4} e_{6}^{2} f_{1}^{4} f_{2} f_{5}-2 b_{4}^{4} e_{6}^{2} f_{1}^{2} f_{2}^{3} f_{5}+b_{4}^{3} e_{6}^{3} f_{1}^{2} f_{2}^{2} f_{3}^{2}$ $+4 b_{4}^{3} e_{6}^{2} e_{7} f_{1}^{4} f_{2}^{2}+2 b_{4}^{3} e_{6}^{2} e_{7} f_{1}^{2} f_{2}^{4}+a_{6} b_{4}^{5} e_{6} f_{1}^{4} f_{2}^{2}-b_{1}^{5} b_{4} f_{2}^{2} f_{3} f_{5}^{3}+b_{1}^{5} e_{7} f_{2}^{3} f_{3} f_{5}^{2}-b_{1}^{4}$ $b_{4}^{2} f_{1}^{2} f_{2} f_{5}^{3}+b_{1}^{4} b_{4} e_{7} f_{2}^{4} f_{5}^{2}+b_{1}^{3} e_{6}^{2} e_{7} f_{2}^{3} f_{3}^{3}+2 b_{1}^{3} e_{6} e_{7}^{2} f_{2}^{5} f_{3}+b_{1}^{2} b_{4}^{3} e_{6} f_{1}^{4} f_{5}^{2}+2 b_{1}^{2} b_{4}^{3} e_{6}$ $f_{2}^{4} f_{5}^{2}+b_{1}^{2} b_{4} e_{6}^{3} f_{2}^{2} f_{3}^{4}+2 b_{1}^{2} b_{4} e_{6} e_{7}^{2} f_{2}^{6}-2 b_{1} b_{4}^{3} e_{6}^{2} f_{1}^{5} f_{7}+2 b_{1} b_{4}^{2} e_{6}^{3} f_{2}^{3} f_{3}^{3}+a_{6} b_{4}^{5} e_{6} f_{1}^{6}$ $\left.-b_{1}^{4} b_{4}^{2} f_{2}^{3} f_{5}^{3}+b_{4}^{3} e_{6}^{3} f_{2}^{4} f_{3}^{2}+2 b_{4}^{3} e_{6}^{2} e_{7} f_{1}^{6}\right) X_{3}^{3}+\left(-5 b_{1}^{3} b_{4}^{2} e_{6} f_{1} f_{2} f_{3}^{2} f_{5} f_{7}-b_{1}^{3} b_{4} e_{6} e_{7} f_{1}\right.$ $f_{2}^{2} f_{3}^{2} f_{7}-4 b_{1}^{2} b_{4}^{3} e_{6} f_{1} f_{2}^{2} f_{3} f_{5} f_{7}-6 b_{1}^{2} b_{4}^{2} e_{6} e_{7} f_{1}^{3} f_{2} f_{3} f_{7}-2 b_{1}^{2} b_{4}^{2} e_{6} e_{7} f_{1}^{2} f_{2} f_{3}^{2} f_{5}-8 b_{1}$ $b_{4}^{3} e_{6} e_{7} f_{1}^{2} f_{2}^{2} f_{3} f_{5}+3 b_{1}^{4} b_{4} e_{7} f_{2}^{2} f_{3}^{2} f_{5}^{2}-2 b_{1}^{4} e_{7}^{2} f_{1} f_{2}^{3} f_{3} f_{7}-b_{1}^{3} b_{4}^{2} e_{6} f_{2}^{3} f_{3} f_{7}^{2}+2 b_{1}^{3} b_{4}^{2} e_{6} f_{2}$ $f_{3}^{3} f_{5}^{2}+6 b_{1}^{3} b_{4}^{2} e_{7} f_{2}^{3} f_{3} f_{5}^{2}+b_{1}^{5} b_{4} f_{2}^{2} f_{3} f_{5} f_{7}^{2}-b_{1}^{4} b_{4}^{2} f_{1}^{2} f_{2} f_{5} f_{7}^{2}+3 b_{1}^{4} b_{4} e_{7} f_{1}^{2} f_{2}^{2} f_{7}^{2}-3$ $b_{1}^{3} b_{4} e_{7}^{2} f_{1}^{3} f_{2}^{2} f_{7}-3 b_{1}^{3} b_{4} e_{7}^{2} f_{1} f_{2}^{4} f_{7}-5 b_{1}^{3} b_{4} e_{7}^{2} f_{2}^{4} f_{3} f_{5}+b_{1}^{2} b_{4}^{3} e_{6} f_{1}^{2} f_{3}^{2} f_{5}^{2}+4 b_{1}^{2} b_{4}^{3} e_{6} f_{2}^{2}$ $f_{3}^{2} f_{5}^{2}+3 b_{1}^{2} b_{4}^{3} e_{7} f_{1}^{2} f_{2}^{2} f_{5}^{2}-b_{1}^{2} b_{4}^{2} e_{6}^{2} f_{2} f_{3}^{4} f_{5}-b_{1}^{2} b_{4}^{2} e_{7}^{2} f_{1}^{4} f_{2} f_{5}-4 b_{1}^{2} b_{4}^{2} e_{7}^{2} f_{1}^{2} f_{2}^{3} f_{5}+b_{1}^{2} b_{4}$ $e_{6}^{2} e_{7} f_{2}^{2} f_{3}^{4}+3 b_{1}^{2} b_{4} e_{6} e_{7}^{2} f_{2}^{4} f_{3}^{2}+2 b_{1} b_{4}^{4} e_{6} f_{2}^{3} f_{3} f_{5}^{2}-2 b_{1} b_{4}^{3} e_{6}^{2} f_{2}^{2} f_{3}^{3} f_{5}-2 b_{1} b_{4}^{3} e_{6} e_{7}$ $f_{1}^{5} f_{7}+2 b_{1} b_{4}^{2} e_{6}^{2} e_{7} f_{2}^{3} f_{3}^{3}+2 b_{1} b_{4}^{2} e_{6} e_{7}^{2} f_{2}^{5} f_{3}+b_{4}^{4} e_{6}^{2} f_{1}^{3} f_{2} f_{3} f_{7}-b_{4}^{4} e_{6}^{2} f_{1}^{2} f_{2} f_{3}^{2} f_{5}+b_{4}^{4}$ $e_{6}^{2} f_{1} f_{2}^{3} f_{3} f_{7}-2 b_{4}^{4} e_{6} e_{7} f_{1}^{4} f_{2} f_{5}-2 b_{4}^{4} e_{6} e_{7} f_{1}^{2} f_{2}^{3} f_{5}+b_{4}^{3} e_{6}^{2} e_{7} f_{1}^{2} f_{2}^{2} f_{3}^{2}+a_{7} b_{1} b_{4}^{4} e_{6} f_{1}^{2} f_{2}^{3} f_{3}$ $-a_{7} b_{1}^{3} b_{4}^{3} f_{1}^{2} f_{2}^{2} f_{3} f_{5}+a_{7} b_{1}^{3} b_{4}^{2} e_{7} f_{1}^{2} f_{2}^{3} f_{3}+3 a_{7} b_{1} b_{4}^{4} e_{6} f_{1}^{4} f_{2} f_{3}-a_{7} b_{1}^{2} b_{4}^{3} e_{6} f_{2}^{4} f_{3}^{2}+a_{7}$ $b_{1}^{2} b_{4}^{3} e_{7} f_{1}^{4} f_{2}^{2}+a_{7} b_{1}^{2} b_{4}^{3} e_{7} f_{1}^{2} f_{2}^{4}+2 b_{1}^{4} b_{4}^{2} f_{1} f_{2} f_{3} f_{5}^{2} f_{7}+3 b_{1}^{3} b_{4}^{2} e_{6} f_{1}^{2} f_{2} f_{3} f_{7}^{2}+2 b_{1}^{3} b_{4}^{2} e_{7}$ $f_{1}^{3} f_{2} f_{5} f_{7}+2 b_{1}^{3} b_{4}^{2} e_{7} f_{1} f_{2}^{3} f_{5} f_{7}-4 b_{1}^{3} b_{4} e_{6} e_{7} f_{2}^{2} f_{3}^{3} f_{5}-b_{1}^{3} b_{4} e_{7}^{2} f_{1}^{2} f_{2}^{2} f_{3} f_{5}-2 b_{1}^{2} b_{4}^{3} e_{6}$ $f_{1}^{3} f_{3} f_{5} f_{7}+b_{1}^{2} b_{4}^{2} e_{6}^{2} f_{1} f_{2} f_{3}^{3} f_{7}-8 b_{1}^{2} b_{4}^{2} e_{6} e_{7} f_{2}^{3} f_{3}^{2} f_{5}+b_{1}^{2} b_{4} e_{6} e_{7}^{2} f_{1}^{2} f_{2}^{2} f_{3}^{2}-b_{1} b_{4}^{4} e_{6}$ $f_{1}^{3} f_{2} f_{5} f_{7}+4 b_{1} b_{4}^{4} e_{6} f_{1}^{2} f_{2} f_{3} f_{5}^{2}-b_{1} b_{4}^{4} e_{6} f_{1} f_{2}^{3} f_{5} f_{7}+2 b_{1} b_{4}^{3} e_{6}^{2} f_{1} f_{2}^{2} f_{3}^{2} f_{7}-b_{1} b_{4}^{3} e_{6} e_{7} f_{1}^{3}$ $f_{2}^{2} f_{7}+b_{1} b_{4}^{3} e_{6} e_{7} f_{1} f_{2}^{4} f_{7}-4 b_{1} b_{4}^{3} e_{6} e_{7} f_{2}^{4} f_{3} f_{5}+3 b_{1} b_{4}^{2} e_{6} e_{7}^{2} f_{1}^{4} f_{2} f_{3}+5 b_{1} b_{4}^{2} e_{6} e_{7}^{2} f_{1}^{2}$ $f_{2}^{3} f_{3}+a_{7} b_{1}^{4} b_{4}^{2} f_{2}^{3} f_{3}^{2} f_{5}-a_{7} b_{1}^{4} b_{4} e_{7} f_{2}^{4} f_{3}^{2}-a_{7} b_{1}^{3} b_{4}^{3} f_{1}^{3} f_{2}^{2} f_{7}-a_{7} b_{1}^{3} b_{4}^{3} f_{1} f_{2}^{4} f_{7}+a_{7} b_{1}^{3} b_{4}^{3}$ 
$f_{2}^{4} f_{3} f_{5}-a_{7} b_{1}^{3} b_{4}^{2} e_{6} f_{2}^{3} f_{3}^{3}-a_{7} b_{1}^{3} b_{4}^{2} e_{7} f_{2}^{5} f_{3}-a_{7} b_{1}^{2} b_{4}^{4} f_{1}^{4} f_{2} f_{5}-a_{7} b_{1}^{2} b_{4}^{4} f_{1}^{2} f_{2}^{3} f_{5}+a_{7}$ $b_{4}^{5} e_{6} f_{1}^{4} f_{2}^{2}-b_{1}^{5} b_{4} f_{1} f_{2}^{2} f_{7}^{3}+b_{1}^{5} e_{7} f_{2}^{3} f_{3} f_{7}^{2}+b_{1}^{4} b_{4}^{2} f_{2}^{3} f_{5} f_{7}^{2}-b_{1}^{4} b_{4}^{2} f_{2} f_{3}^{2} f_{5}^{3}+b_{1}^{4} b_{4} e_{7} f_{2}^{4} f_{7}^{2}$ $-2 b_{1}^{4} e_{7}^{2} f_{2}^{3} f_{3}^{2} f_{5}-2 b_{1}^{3} b_{4}^{3} f_{2}^{2} f_{3} f_{5}^{3}+b_{1}^{3} e_{6} e_{7}^{2} f_{2}^{3} f_{3}^{3}+b_{1}^{3} e_{7}^{3} f_{1}^{2} f_{2}^{3} f_{3}-b_{1}^{2} b_{4}^{4} f_{1}^{2} f_{2} f_{5}^{3}+b_{1}^{2}$ $b_{4}^{3} e_{6} f_{1}^{4} f_{7}^{2}-b_{1}^{2} b_{4}^{3} e_{6} f_{2}^{4} f_{7}^{2}+3 b_{1}^{2} b_{4}^{3} e_{7} f_{2}^{4} f_{5}^{2}-3 b_{1}^{2} b_{4}^{2} e_{7}^{2} f_{2}^{5} f_{5}+b_{1}^{2} b_{4} e_{7}^{3} f_{1}^{4} f_{2}^{2}+2 b_{1}^{2} b_{4}$ $e_{7}^{3} f_{1}^{2} f_{2}^{4}+b_{4}^{5} e_{6} f_{1}^{2} f_{2}^{2} f_{5}^{2}-b_{4}^{4} e_{6}^{2} f_{2}^{3} f_{3}^{2} f_{5}+b_{4}^{3} e_{6}^{2} e_{7} f_{2}^{4} f_{3}^{2}+2 b_{4}^{3} e_{6} e_{7}^{2} f_{1}^{4} f_{2}^{2}+b_{4}^{3} e_{6} e_{7}^{2} f_{1}^{2} f_{2}^{4}$ $\left.+a_{7} b_{4}^{5} e_{6} f_{1}^{6}+b_{1}^{3} e_{7}^{3} f_{2}^{5} f_{3}-b_{1}^{2} b_{4}^{4} f_{2}^{3} f_{5}^{3}+b_{1}^{2} b_{4} e_{7}^{3} f_{2}^{6}+b_{4}^{5} e_{6} f_{1}^{4} f_{5}^{2}+b_{4}^{3} e_{6} e_{7}^{2} f_{1}^{6}\right) X_{3}$ $+\left(6 b_{1}^{3} b_{4}^{2} e_{6} f_{1}^{2} f_{2} f_{3} f_{5} f_{7}-2 b_{1}^{3} b_{4} e_{6} e_{7} f_{1}^{2} f_{2}^{2} f_{3} f_{7}-6 b_{1}^{3} b_{4} e_{6} e_{7} f_{1} f_{2}^{2} f_{3}^{2} f_{5}-8 b_{1}^{2}\right.$ $b_{4}^{2} e_{6} e_{7} f_{1}^{3} f_{2} f_{3} f_{5}-4 b_{1}^{2} b_{4}^{2} e_{6} e_{7} f_{1} f_{2}^{3} f_{3} f_{5}-b_{1}^{3} b_{4} e_{6}^{2} f_{2}^{2} f_{3}^{3} f_{7}-2 b_{1}^{3} b_{4} e_{7}^{2} f_{1}^{3} f_{2}^{2} f_{5}-2 b_{1}^{3} b_{4}$ $e_{7}^{2} f_{1} f_{2}^{4} f_{5}+4 b_{1}^{3} e_{6} e_{7}^{2} f_{1} f_{2}^{3} f_{3}^{2}+2 b_{1}^{2} b_{4}^{3} e_{6} f_{1}^{4} f_{5} f_{7}-b_{1}^{2} b_{4}^{3} e_{6} f_{1}^{3} f_{3} f_{5}^{2}-2 b_{1}^{2} b_{4}^{2} e_{6}^{2} f_{2}^{3} f_{3}^{2} f_{7}$ $-b_{1} b_{4}^{3} e_{6}^{2} f_{2}^{4} f_{3} f_{7}-2 b_{1} b_{4}^{3} e_{6} e_{7} f_{1}^{5} f_{5}+b_{4}^{4} e_{6}^{2} f_{1}^{3} f_{2} f_{3} f_{5}+b_{4}^{4} e_{6}^{2} f_{1} f_{2}^{3} f_{3} f_{5}+b_{4}^{3} e_{6}^{2} e_{7} f_{1}^{3}$ $f_{2}^{2} f_{3}-b_{4}^{3} e_{6}^{2} e_{7} f_{1} f_{2}^{4} f_{3}-a_{6} b_{1}^{4} b_{4}^{2} f_{2}^{3} f_{3}^{2} f_{7}-a_{6} b_{1}^{3} b_{4}^{3} f_{2}^{4} f_{3} f_{7}+a_{6} b_{4}^{5} e_{6} f_{1}^{3} f_{2}^{2} f_{3}-2 b_{1}^{5} b_{4} f_{1}$ $f_{2}^{2} f_{5} f_{7}^{2}-b_{1}^{5} b_{4} f_{2}^{2} f_{3} f_{5}^{2} f_{7}+2 b_{1}^{5} e_{7} f_{2}^{3} f_{3} f_{5} f_{7}-2 b_{1}^{4} b_{4}^{2} f_{1}^{2} f_{2} f_{5}^{2} f_{7}+b_{1}^{4} b_{4}^{2} f_{1} f_{2} f_{3} f_{5}^{3}+2$ $b_{1}^{4} b_{4} e_{7} f_{2}^{4} f_{5} f_{7}-2 b_{1}^{4} e_{6} e_{7} f_{2}^{3} f_{3}^{2} f_{7}-2 b_{1}^{4} e_{7}^{2} f_{1} f_{2}^{3} f_{3} f_{5}+2 b_{1}^{3} b_{4}^{2} e_{7} f_{1}^{3} f_{2} f_{5}^{2}+2 b_{1}^{3} b_{4}^{2} e_{7} f_{1}$ $f_{2}^{3} f_{5}^{2}-a_{6} b_{1}^{3} b_{4}^{3} f_{1}^{2} f_{2}^{2} f_{3} f_{7}-2 a_{6} b_{1}^{3} b_{4}^{3} f_{1} f_{2}^{2} f_{3}^{2} f_{5}+2 a_{6} b_{1}^{3} b_{4}^{2} e_{7} f_{1} f_{2}^{3} f_{3}^{2}-a_{6} b_{1}^{2} b_{4}^{4}$ $f_{1}^{3} f_{2} f_{3} f_{5}+3 a_{7} b_{1}^{4} b_{4}^{2} f_{1} f_{2}^{3} f_{3} f_{5}-3 a_{7} b_{1}^{4} b_{4} e_{7} f_{1} f_{2}^{4} f_{3}-4 a_{7} b_{1}^{3} b_{4}^{2} e_{6} f_{1} f_{2}^{3} f_{3}^{2}-7 a_{7} b_{1}^{2}$ $b_{4}^{3} e_{6} f_{1}^{3} f_{2}^{2} f_{3}-3 a_{7} b_{1}^{2} b_{4}^{3} e_{6} f_{1} f_{2}^{4} f_{3}+2 b_{1}^{2} b_{4} e_{6} e_{7}^{2} f_{1} f_{2}^{4} f_{3}-3 b_{1} b_{4}^{3} e_{6}^{2} f_{1}^{2} f_{2}^{2} f_{3} f_{7}+2 b_{1} b_{4}^{3}$ $e_{6}^{2} f_{1} f_{2}^{2} f_{3}^{2} f_{5}-2 b_{1} b_{4}^{3} e_{6} e_{7} f_{1}^{3} f_{2}^{2} f_{5}+8 b_{1} b_{4}^{2} e_{6}^{2} e_{7} f_{1}^{3} f_{2} f_{3}^{2}+2 b_{1} b_{4}^{2} e_{6}^{2} e_{7} f_{1} f_{2}^{3} f_{3}^{2}-a_{6} b_{1}^{2}$ $b_{4}^{4} f_{1} f_{2}^{3} f_{3} f_{5}+3 a_{6} b_{1}^{2} b_{4}^{3} e_{6} f_{1} f_{2}^{2} f_{3}^{3}+a_{6} b_{1}^{2} b_{4}^{3} e_{7} f_{1}^{3} f_{2}^{2} f_{3}+a_{6} b_{1}^{2} b_{4}^{3} e_{7} f_{1} f_{2}^{4} f_{3}+4 a_{6} b_{1}$ $b_{4}^{4} e_{6} f_{1}^{3} f_{2} f_{3}^{2}+2 a_{6} b_{1} b_{4}^{4} e_{6} f_{1} f_{2}^{3} f_{3}^{2}+2 b_{1}^{4} b_{4} e_{6} f_{2}^{2} f_{3}^{2} f_{5} f_{7}+4 b_{1}^{4} b_{4} e_{7} f_{1}^{2} f_{2}^{2} f_{5} f_{7}+$ $b_{1}^{4} b_{4} e_{7} f_{1} f_{2}^{2} f_{3} f_{5}^{2}-2 b_{1}^{3} b_{4}^{2} e_{6} f_{1} f_{2} f_{3}^{2} f_{5}^{2}+2 b_{1}^{3} b_{4}^{2} e_{6} f_{2}^{3} f_{3} f_{5} f_{7}-2 b_{1}^{3} b_{4} e_{6} e_{7} f_{2}^{4} f_{3} f_{7}+2$ $b_{1}^{2} b_{4}^{3} e_{6} f_{1}^{2} f_{2}^{2} f_{5} f_{7}-b_{1}^{2} b_{4}^{3} e_{6} f_{1} f_{2}^{2} f_{3} f_{5}^{2}-b_{1}^{2} b_{4}^{2} e_{6}^{2} f_{1}^{2} f_{2} f_{3}^{2} f_{7}+b_{1}^{2} b_{4}^{2} e_{6}^{2} f_{1} f_{2} f_{3}^{3} f_{5}+5 b_{1}^{2} b_{4}$ $e_{6}^{2} e_{7} f_{1} f_{2}^{2} f_{3}^{3}+2 b_{1}^{2} b_{4} e_{6} e_{7}^{2} f_{1}^{3} f_{2}^{2} f_{3}+a_{7} b_{1}^{5} b_{4} f_{2}^{4} f_{3} f_{7}+2 a_{7} b_{1}^{4} b_{4}^{2} f_{1}^{2} f_{2}^{3} f_{7}+2 a_{7} b_{1}^{3} b_{4}^{3} f_{1}^{3}$ $f_{2}^{2} f_{5}+2 a_{7} b_{1}^{3} b_{4}^{3} f_{1} f_{2}^{4} f_{5}-2 a_{7} b_{1}^{3} b_{4}^{2} e_{7} f_{1}^{3} f_{2}^{3}-2 a_{7} b_{1}^{3} b_{4}^{2} e_{7} f_{1} f_{2}^{5}-2 a_{7} b_{1} b_{4}^{4} e_{6} f_{1}^{5} f_{2}$ $-2 a_{7} b_{1} b_{4}^{4} e_{6} f_{1}^{3} f_{2}^{3}+a_{7} b_{1}^{4} b_{4}^{2} f_{2}^{5} f_{7}+a_{6} b_{4}^{5} e_{6} f_{1}^{5} f_{3}-b_{1}^{4} b_{4}^{2} f_{2}^{3} f_{5}^{2} f_{7}-b_{4}^{4} e_{6}^{2} f_{1}^{4} f_{2} f_{7}-b_{4}^{4}$ $\left.e_{6}^{2} f_{1}^{2} f_{2}^{3} f_{7}+2 b_{4}^{3} e_{6}^{2} e_{7} f_{1}^{5} f_{3}\right) X_{3}^{2}+\left(2 b_{1}^{4} b_{4} e_{7} f_{2}^{2} f_{3}^{3} f_{5}+2 b_{1}^{3} b_{4}^{2} e_{6} f_{2} f_{3}^{4} f_{5}+4 b_{1}^{3} b_{4}^{2} e_{7} f_{2}^{3}\right.$ $f_{3}^{2} f_{5}-2 b_{1}^{3} b_{4} e_{6} e_{7} f_{2}^{2} f_{3}^{4}+b_{1}^{2} b_{4}^{4} f_{1}^{3} f_{2} f_{5} f_{7}-b_{1}^{2} b_{4}^{4} f_{1}^{2} f_{2} f_{3} f_{5}^{2}+b_{1}^{2} b_{4}^{4} f_{1} f_{2}^{3} f_{5} f_{7}-b_{1}^{2} b_{4}^{3} e_{6}$ $f_{1}^{3} f_{3}^{2} f_{7}+b_{1}^{2} b_{4}^{3} e_{6} f_{1}^{2} f_{3}^{3} f_{5}+4 b_{1}^{2} b_{4}^{3} e_{6} f_{2}^{2} f_{3}^{3} f_{5}-b_{1}^{2} b_{4}^{3} e_{7} f_{1}^{3} f_{2}^{2} f_{7}-b_{1}^{2} b_{4}^{3} e_{7} f_{1} f_{2}^{4} f_{7}+2 b_{1}^{2}$ 
$b_{4}^{3} e_{7} f_{2}^{4} f_{3} f_{5}-4 b_{1}^{2} b_{4}^{2} e_{6} e_{7} f_{2}^{3} f_{3}^{3}-b_{1}^{2} b_{4}^{2} e_{7}^{2} f_{1}^{2} f_{2}^{3} f_{3}+2 b_{1} b_{4}^{4} e_{6} f_{2}^{3} f_{3}^{2} f_{5}-2 b_{1} b_{4}^{3} e_{6} e_{7} f_{2}^{4}$ $f_{3}^{2}+b_{4}^{5} e_{6} f_{1}^{2} f_{2}^{2} f_{3} f_{5}-b_{4}^{4} e_{6} e_{7} f_{1}^{4} f_{2} f_{3}-b_{4}^{4} e_{6} e_{7} f_{1}^{2} f_{2}^{3} f_{3}+b_{1}^{4} b_{4}^{2} f_{1} f_{2} f_{3}^{2} f_{5} f_{7}-b_{1}^{4} b_{4} e_{7} f_{1}$ $f_{2}^{2} f_{3}^{2} f_{7}+2 b_{1}^{3} b_{4}^{3} f_{1} f_{2}^{2} f_{3} f_{5} f_{7}-3 b_{1}^{3} b_{4}^{2} e_{6} f_{1} f_{2} f_{3}^{3} f_{7}-2 b_{1}^{3} b_{4}^{2} e_{7} f_{1} f_{2}^{3} f_{3} f_{7}-6 b_{1}^{2} b_{4}^{3} e_{6} f_{1} f_{2}^{2}$ $f_{3}^{2} f_{7}+2 b_{1}^{2} b_{4}^{3} e_{7} f_{1}^{2} f_{2}^{2} f_{3} f_{5}-b_{1}^{2} b_{4}^{2} e_{6} e_{7} f_{1}^{2} f_{2} f_{3}^{3}-5 b_{1} b_{4}^{4} e_{6} f_{1}^{3} f_{2} f_{3} f_{7}+4 b_{1} b_{4}^{4} e_{6} f_{1}^{2} f_{2}$ $f_{3}^{2} f_{5}-3 b_{1} b_{4}^{4} e_{6} f_{1} f_{2}^{3} f_{3} f_{7}-4 b_{1} b_{4}^{3} e_{6} e_{7} f_{1}^{2} f_{2}^{2} f_{3}^{2}+b_{1}^{5} b_{4} f_{2}^{2} f_{3}^{2} f_{7}^{2}+2 b_{1}^{4} b_{4}^{2} f_{2}^{3} f_{3} f_{7}^{2}-b_{1}^{4}$ $b_{4}^{2} f_{2} f_{3}^{3} f_{5}^{2}+b_{1}^{3} b_{4}^{3} f_{1}^{2} f_{2}^{2} f_{7}^{2}-2 b_{1}^{3} b_{4}^{3} f_{2}^{2} f_{3}^{2} f_{5}^{2}-2 b_{1}^{3} b_{4} e_{7}^{2} f_{2}^{4} f_{3}^{2}-b_{1}^{2} b_{4}^{4} f_{2}^{3} f_{3} f_{5}^{2}-b_{1}^{2} b_{4}^{2}$ $e_{6}^{2} f_{2} f_{3}^{5}-b_{1}^{2} b_{4}^{2} e_{7}^{2} f_{2}^{5} f_{3}-2 b_{1} b_{4}^{3} e_{6}^{2} f_{2}^{2} f_{3}^{4}+b_{4}^{5} e_{6} f_{1}^{4} f_{3} f_{5}-b_{4}^{5} e_{6} f_{1}^{3} f_{2}^{2} f_{7}-b_{4}^{4} e_{6}^{2} f_{1}^{2} f_{2} f_{3}^{3}-$ $\left.b_{1}^{4} e_{7}^{2} f_{2}^{3} f_{3}^{3}+b_{1}^{3} b_{4}^{3} f_{2}^{4} f_{7}^{2}-b_{4}^{5} e_{6} f_{1}^{5} f_{7}-b_{4}^{4} e_{6}^{2} f_{2}^{3} f_{3}^{3}\right) X_{1}+\left(-2 a_{6} b_{1} b_{4}^{4} e_{6} f_{1}^{5} f_{2}-2 a_{6} b_{1}\right.$ $b_{4}^{4} e_{6} f_{1}^{3} f_{2}^{3}+2 b_{1}^{3} b_{4}^{2} e_{6} f_{1}^{3} f_{2} f_{5}^{2}+2 b_{1}^{3} b_{4}^{2} e_{6} f_{1} f_{2}^{3} f_{5}^{2}+b_{1}^{3} b_{4} e_{6}^{2} f_{2}^{4} f_{3} f_{7}+2 b_{1}^{3} e_{6}^{2} e_{7} f_{1} f_{2}^{3} f_{3}^{2}$ $+b_{1}^{2} b_{4}^{2} e_{6}^{2} f_{1}^{2} f_{2}^{3} f_{7}+2 b_{1}^{2} b_{4} e_{6}^{3} f_{1} f_{2}^{2} f_{3}^{3}-2 b_{1} b_{4}^{3} e_{6}^{2} f_{1}^{3} f_{2}^{2} f_{5}+4 b_{1} b_{4}^{2} e_{6}^{3} f_{1}^{3} f_{2} f_{3}^{2}+3 a_{6} b_{1}^{4}$ $b_{4}^{2} f_{1} f_{2}^{3} f_{3} f_{5}-3 a_{6} b_{1}^{4} b_{4} e_{7} f_{1} f_{2}^{4} f_{3}-4 a_{6} b_{1}^{3} b_{4}^{2} e_{6} f_{1} f_{2}^{3} f_{3}^{2}-7 a_{6} b_{1}^{2} b_{4}^{3} e_{6} f_{1}^{3} f_{2}^{2} f_{3}-3 a_{6} b_{1}^{2}$ $b_{4}^{3} e_{6} f_{1} f_{2}^{4} f_{3}+2 b_{1}^{4} b_{4} e_{6} f_{1}^{2} f_{2}^{2} f_{5} f_{7}+2 b_{1}^{4} b_{4} e_{6} f_{1} f_{2}^{2} f_{3} f_{5}^{2}-2 b_{1}^{4} e_{6} e_{7} f_{1} f_{2}^{3} f_{3} f_{5}-b_{1}^{3} b_{4} e_{6}^{2}$ $f_{1}^{2} f_{2}^{2} f_{3} f_{7}-4 b_{1}^{3} b_{4} e_{6}^{2} f_{1} f_{2}^{2} f_{3}^{2} f_{5}-2 b_{1}^{3} b_{4} e_{6} e_{7} f_{1}^{3} f_{2}^{2} f_{5}-2 b_{1}^{3} b_{4} e_{6} e_{7} f_{1} f_{2}^{4} f_{5}-7 b_{1}^{2} b_{4}^{2} e_{6}^{2}$ $f_{1}^{3} f_{2} f_{3} f_{5}-3 b_{1}^{2} b_{4}^{2} e_{6}^{2} f_{1} f_{2}^{3} f_{3} f_{5}+b_{1}^{2} b_{4} e_{6}^{2} e_{7} f_{1}^{3} f_{2}^{2} f_{3}+b_{1}^{2} b_{4} e_{6}^{2} e_{7} f_{1} f_{2}^{4} f_{3}+a_{6} b_{1}^{5} b_{4} f_{2}^{4} f_{3} f_{7}$ $+2 a_{6} b_{1}^{4} b_{4}^{2} f_{1}^{2} f_{2}^{3} f_{7}+2 a_{6} b_{1}^{3} b_{4}^{3} f_{1}^{3} f_{2}^{2} f_{5}+2 a_{6} b_{1}^{3} b_{4}^{3} f_{1} f_{2}^{4} f_{5}-2 a_{6} b_{1}^{3} b_{4}^{2} e_{7} f_{1}^{3} f_{2}^{3}-2 a_{6}$ $b_{1}^{3} b_{4}^{2} e_{7} f_{1} f_{2}^{5}+a_{6} b_{1}^{4} b_{4}^{2} f_{2}^{5} f_{7}+b_{1}^{2} b_{4}^{2} e_{6}^{2} f_{2}^{5} f_{7}-2 b_{1} b_{4}^{3} e_{6}^{2} f_{1}^{5} f_{5}-b_{4}^{3} e_{6}^{3} f_{1} f_{2}^{4} f_{3}+b_{4}^{3} e_{6}^{3}$ $\left.f_{1}^{5} f_{3}\right) X_{3}^{4}+\left(3 a_{6} b_{1}^{3} b_{4}^{2} e_{6} f_{1}^{2} f_{2}^{3} f_{3}-b_{1}^{3} b_{4} e_{6}^{2} f_{1}^{2} f_{2}^{2} f_{3} f_{5}-a_{6} b_{1}^{5} b_{4} f_{1} f_{2}^{4} f_{7}-a_{6} b_{1}^{5} b_{4} f_{2}^{4} f_{3} f_{5}\right.$ $-a_{6} b_{1}^{4} b_{4}^{2} f_{1}^{2} f_{2}^{3} f_{5}+a_{6} b_{1}^{4} b_{4} e_{6} f_{2}^{4} f_{3}^{2}+a_{6} b_{1}^{4} b_{4} e_{7} f_{1}^{2} f_{2}^{4}+a_{6} b_{1}^{3} b_{4}^{2} e_{6} f_{2}^{5} f_{3}+a_{6} b_{1}^{2} b_{4}^{3} e_{6}$ $f_{1}^{4} f_{2}^{2}+a_{6} b_{1}^{2} b_{4}^{3} e_{6} f_{1}^{2} f_{2}^{4}-b_{1}^{3} b_{4} e_{6}^{2} f_{1}^{3} f_{2}^{2} f_{7}-b_{1}^{3} b_{4} e_{6}^{2} f_{1} f_{2}^{4} f_{7}-b_{1}^{3} b_{4} e_{6}^{2} f_{2}^{4} f_{3} f_{5}+b_{1}^{3} e_{6}^{2} e_{7}$ $f_{1}^{2} f_{2}^{3} f_{3}-b_{1}^{2} b_{4}^{2} e_{6}^{2} f_{1}^{4} f_{2} f_{5}-2 b_{1}^{2} b_{4}^{2} e_{6}^{2} f_{1}^{2} f_{2}^{3} f_{5}+b_{1}^{2} b_{4} e_{6}^{3} f_{1}^{2} f_{2}^{2} f_{3}^{2}+b_{1}^{2} b_{4} e_{6}^{2} e_{7} f_{1}^{4} f_{2}^{2}+2$ $b_{1}^{2} b_{4} e_{6}^{2} e_{7} f_{1}^{2} f_{2}^{4}+3 b_{1} b_{4}^{2} e_{6}^{3} f_{1}^{4} f_{2} f_{3}+4 b_{1} b_{4}^{2} e_{6}^{3} f_{1}^{2} f_{2}^{3} f_{3}-b_{1}^{2} b_{4}^{2} e_{6}^{2} f_{2}^{5} f_{5}+b_{1}^{2} b_{4} e_{6}^{3} f_{2}^{4} f_{3}^{2}+$ $b_{1}^{2} b_{4} e_{6}^{2} e_{7} f_{2}^{6}+b_{1} b_{4}^{2} e_{6}^{3} f_{2}^{5} f_{3}+a_{6} b_{1}^{5} e_{7} f_{2}^{5} f_{3}-a_{6} b_{1}^{4} b_{4}^{2} f_{2}^{5} f_{5}+a_{6} b_{1}^{4} b_{4} e_{7} f_{2}^{6}+b_{1}^{3} e_{6}^{2} e_{7}$ $\left.f_{2}^{5} f_{3}+2 b_{4}^{3} e_{6}^{3} f_{1}^{4} f_{2}^{2}+b_{4}^{3} e_{6}^{3} f_{1}^{2} f_{2}^{4}+b_{4}^{3} e_{6}^{3} f_{1}^{6}\right) X_{3}^{5}-b_{1}^{2} b_{4}^{2} e_{6} e_{7} f_{1}^{2} f_{2} f_{3}^{2} f_{7}+4 b_{1} b_{4}^{4} e_{6}$ $f_{1}^{2} f_{2} f_{3} f_{5} f_{7}-4 b_{1} b_{4}^{3} e_{6} e_{7} f_{1}^{2} f_{2}^{2} f_{3} f_{7}-b_{1}^{2} b_{4}^{3} e_{6} f_{1}^{3} f_{3} f_{7}^{2}+2 b_{1}^{2} b_{4}^{3} e_{7} f_{2}^{4} f_{5} f_{7}-b_{1}^{2} b_{4}^{2} e_{6}^{2} f_{2}$ $f_{3}^{4} f_{7}-b_{1}^{2} b_{4}^{2} e_{7}^{2} f_{1}^{2} f_{2}^{3} f_{7}+b_{1}^{2} b_{4} e_{7}^{3} f_{1}^{3} f_{2}^{2} f_{3}+b_{1}^{2} b_{4} e_{7}^{3} f_{1} f_{2}^{4} f_{3}-b_{1} b_{4}^{4} e_{6} f_{1}^{3} f_{2} f_{7}^{2}-b_{1}$ $b_{4}^{4} e_{6} f_{1} f_{2}^{3} f_{7}^{2}-2 b_{1} b_{4}^{3} e_{6}^{2} f_{2}^{2} f_{3}^{3} f_{7}+b_{4}^{5} e_{6} f_{1}^{2} f_{2}^{2} f_{5} f_{7}-b_{4}^{4} e_{6}^{2} f_{1}^{2} f_{2} f_{3}^{2} f_{7}-b_{4}^{4} e_{6} e_{7} f_{1}^{4} f_{2} f_{7}-$ $b_{4}^{4} e_{6} e_{7} f_{1}^{2} f_{2}^{3} f_{7}+b_{4}^{3} e_{6} e_{7}^{2} f_{1}^{3} f_{2}^{2} f_{3}-b_{1}^{4} b_{4}^{2} f_{2} f_{3}^{2} f_{5}^{2} f_{7}-2 b_{1}^{3} b_{4}^{3} f_{2}^{2} f_{3} f_{5}^{2} f_{7}-3 b_{1}^{3} b_{4} e_{7}^{2} f_{2}^{4} f_{3} f_{7}$ 


$$
\begin{aligned}
& -b_{1}^{2} b_{4}^{4} f_{1}^{2} f_{2} f_{5}^{2} f_{7}+3 b_{1}^{2} b_{4} e_{6} e_{7}^{2} f_{1} f_{2}^{2} f_{3}^{3}+2 b_{1} b_{4}^{4} e_{6} f_{2}^{3} f_{3} f_{5} f_{7}-2 b_{1} b_{4}^{3} e_{6} e_{7} f_{2}^{4} f_{3} f_{7} \\
& +4 b_{1} b_{4}^{2} e_{6} e_{7}^{2} f_{1}^{3} f_{2} f_{3}^{2}+2 b_{1} b_{4}^{2} e_{6} e_{7}^{2} f_{1} f_{2}^{3} f_{3}^{2}+b_{1}^{4} b_{4}^{2} f_{1} f_{2} f_{3} f_{5} f_{7}^{2}-b_{1}^{4} b_{4} e_{7} f_{1} f_{2}^{2} f_{3} f_{7}^{2} \\
& +2 b_{1}^{4} b_{4} e_{7} f_{2}^{2} f_{3}^{2} f_{5} f_{7}-3 b_{1}^{3} b_{4}^{2} e_{6} f_{1} f_{2} f_{3}^{2} f_{7}^{2}+2 b_{1}^{3} b_{4}^{2} e_{6} f_{2} f_{3}^{3} f_{5} f_{7}+4 b_{1}^{3} b_{4}^{2} e_{7} f_{2}^{3} f_{3} f_{5} f_{7} \\
& -2 b_{1}^{3} b_{4} e_{6} e_{7} f_{2}^{2} f_{3}^{3} f_{7}-b_{1}^{3} b_{4} e_{7}^{2} f_{1}^{2} f_{2}^{2} f_{3} f_{7}-2 b_{1}^{3} b_{4} e_{7}^{2} f_{1} f_{2}^{2} f_{3}^{2} f_{5}+b_{1}^{2} b_{4}^{3} e_{6} f_{1}^{2} f_{3}^{2} f_{5} f_{7} \\
& -3 b_{1}^{2} b_{4}^{3} e_{6} f_{1} f_{2}^{2} f_{3} f_{7}^{2}+4 b_{1}^{2} b_{4}^{3} e_{6} f_{2}^{2} f_{3}^{2} f_{5} f_{7}+2 b_{1}^{2} b_{4}^{3} e_{7} f_{1}^{2} f_{2}^{2} f_{5} f_{7}-4 b_{1}^{2} b_{4}^{2} e_{6} e_{7} f_{2}^{3} f_{3}^{2} f_{7} \\
& -b_{1}^{2} b_{4}^{2} e_{7}^{2} f_{1}^{3} f_{2} f_{3} f_{5}-b_{1}^{2} b_{4}^{2} e_{7}^{2} f_{1} f_{2}^{3} f_{3} f_{5}-a_{7} b_{1}^{4} b_{4}^{2} f_{2}^{3} f_{3}^{2} f_{7}-a_{7} b_{1}^{3} b_{4}^{3} f_{2}^{4} f_{3} f_{7}+a_{7} b_{4}^{5} e_{6} \\
& f_{1}^{3} f_{2}^{2} f_{3}+a_{7} b_{1}^{2} b_{4}^{3} e_{7} f_{1} f_{2}^{4} f_{3}+4 a_{7} b_{1} b_{4}^{4} e_{6} f_{1}^{3} f_{2} f_{3}^{2}+2 a_{7} b_{1} b_{4}^{4} e_{6} f_{1} f_{2}^{3} f_{3}^{2}-a_{7} b_{1}^{3} b_{4}^{3} f_{1}^{2} \\
& f_{2}^{2} f_{3} f_{7}-2 a_{7} b_{1}^{3} b_{4}^{3} f_{1} f_{2}^{2} f_{3}^{2} f_{5}+2 a_{7} b_{1}^{3} b_{4}^{2} e_{7} f_{1} f_{2}^{3} f_{3}^{2}-a_{7} b_{1}^{2} b_{4}^{4} f_{1}^{3} f_{2} f_{3} f_{5}-a_{7} b_{1}^{2} b_{4}^{4} f_{1} \\
& f_{2}^{3} f_{3} f_{5}+3 a_{7} b_{1}^{2} b_{4}^{3} e_{6} f_{1} f_{2}^{2} f_{3}^{3}+a_{7} b_{1}^{2} b_{4}^{3} e_{7} f_{1}^{3} f_{2}^{2} f_{3}+a_{7} b_{4}^{5} e_{6} f_{1}^{5} f_{3}+b_{1}^{5} b_{4} f_{2}^{2} f_{3} f_{7}^{3}-2 b_{1}^{4} \\
& e_{7}^{2} f_{2}^{3} f_{3}^{2} f_{7}+2 b_{1}^{3} e_{7}^{3} f_{1} f_{2}^{3} f_{3}^{2}-b_{1}^{2} b_{4}^{4} f_{2}^{3} f_{5}^{2} f_{7}-b_{1}^{2} b_{4}^{2} e_{7}^{2} f_{2}^{5} f_{7}+b_{4}^{5} e_{6} f_{1}^{4} f_{5} f_{7}-b_{4}^{4} e_{6}^{2} f_{2}^{3} f_{3}^{2} f_{7} \\
& \left.+b_{4}^{3} e_{6} e_{7}^{2} f_{1}^{5} f_{3}+b_{1}^{4} b_{4}^{2} f_{2}^{3} f_{7}^{3}\right]
\end{aligned}
$$




\section{Appendix B}

An example of L-L-L class platform. Deriving univariate polynomial with design parameters as in Example 5.3.2 by Maple. 
with (Groebner);

[Basis, FGLM, HilbertDimension, HilbertPolynomial, HilbertSeries, Homogenize, InitialForm,

InterReduce, IsBasis, Is Proper, IsZeroDimensional, LeadingCoefficient, LeadingMonomial,

LeadingTerm, MatrixOrder, MaximalIndependentSet, MonomialOrder, MultiplicationMatrix,

MultivariateCyclicVector, NormalForm, NormalSet, RationalUnivariateRepresentation,

Reduce, RememberBasis, SPolynomial, Solve, SuggestVariableOrder, Support, TestOrder,

ToricIdealBasis, TrailingTerm, UnivariatePolynomial, Walk, WeightedDegree ]

$$
\begin{aligned}
F:= & {\left[-\frac{\sqrt{3}}{4} \cdot X[1] \cdot X[3]+\frac{1}{4} X[2] \cdot X[3]-\frac{1}{4} \cdot X[1]-\frac{\sqrt{3}}{4} \cdot X[2], \sqrt{2} \cdot X[3]^{2}-\frac{\sqrt{2}}{4} \cdot X[1] \cdot X[3]\right.} \\
& +\frac{\sqrt{2}}{4} \cdot X[2] \cdot X[3]-\frac{\sqrt{2}}{4} \cdot X[1]-\frac{\sqrt{2}}{4} \cdot X[2]+\frac{\sqrt{2}}{2} \cdot X[3]+\frac{\sqrt{2}}{2}, 2 \cdot X[3]^{2}-\frac{1}{2} \cdot X[2] \\
& \left.\cdot X[3]+\frac{1}{2} \cdot X[1]-\frac{1}{2} \cdot X[3]+1\right] \\
{[-} & \frac{1}{4} \sqrt{3} X_{1} X_{3}+\frac{1}{4} X_{2} X_{3}-\frac{1}{4} X_{1}-\frac{1}{4} \sqrt{3} X_{2}, \sqrt{2} X_{3}^{2}-\frac{1}{4} \sqrt{2} X_{1} X_{3}+\frac{1}{4} \sqrt{2} X_{2} X_{3} \\
& -\frac{1}{4} \sqrt{2} X_{1}-\frac{1}{4} \sqrt{2} X_{2}+\frac{1}{2} \sqrt{2} X_{3}+\frac{1}{2} \sqrt{2}, 2 X_{3}^{2}-\frac{1}{2} X_{2} X_{3}+\frac{1}{2} X_{1}-\frac{1}{2} X_{3} \\
& +1] \\
G & =\text { Basis }(F, p l e x(X[1], X[2], X[3])) \\
& {\left[22+44 X_{3}^{2}+(3 \sqrt{3}+7) X_{3},-27 \sqrt{3}-147+(42 \sqrt{3}-66) X_{3}+91 X_{2},-21 \sqrt{3}+33\right.} \\
& \left.+(-54 \sqrt{3}-294) X_{3}+91 X_{1}\right] \\
\text { solve } & \left(22+44 X_{3}^{2}+(3 \sqrt{3}+7) X_{3}, X[3]\right) \\
& -\frac{3}{88} \sqrt{3}-\frac{7}{88}-\frac{1}{88} \cdot \mathrm{I} \sqrt{3796-42 \sqrt{3}},-\frac{3}{88} \sqrt{3}-\frac{7}{88}+\frac{1}{88} \cdot \mathrm{I} \sqrt{3796-42 \sqrt{3}}
\end{aligned}
$$




\section{Appendix C}

An example of T-T-T class platform. Deriving univariate polynomial with design parameters as in Example 5.3.3 by Maple. 
with (Groebner);

[Basis, FGLM, HilbertDimension, HilbertPolynomial, HilbertSeries, Homogenize, InitialForm,

InterReduce, Is Basis, Is Proper, IsZeroDimensional, LeadingCoefficient, LeadingMonomial,

LeadingTerm, MatrixOrder, MaximalIndependentSet, MonomialOrder, MultiplicationMatrix,

MultivariateCyclicVector, NormalForm, NormalSet, RationalUnivariateRepresentation,

Reduce, RememberBasis, SPolynomial, Solve, SuggestVariableOrder, Support, TestOrder,

ToricIdealBasis, TrailingTerm, UnivariatePolynomial, Walk, WeightedDegree ]

$$
\begin{aligned}
F:= & {\left[\frac{-1}{2} \cdot X[1] \cdot X[3]+\frac{1}{2} \cdot X[2],-\frac{1}{2} \cdot X[1]-\frac{1}{2} \cdot X[2] \cdot X[3]+X[3], \frac{2 \cdot \sqrt{3}-5}{8} \cdot X[3]^{2}-\frac{\sqrt{3}}{4}\right.} \\
& \left.\cdot X[1] \cdot X[3]+\frac{1}{4} \cdot X[2] \cdot X[3]+\frac{1}{4} \cdot X[1]+\frac{\sqrt{3}}{4} \cdot X[2]-\frac{(2 \cdot \sqrt{3}+1)}{4} \cdot X[3]-\frac{1}{8}\right] \\
{[-} & \frac{1}{2} X_{1} X_{3}+\frac{1}{2} X_{2},-\frac{1}{2} X_{1}-\frac{1}{2} X_{2} X_{3}+X_{3}, \frac{1}{8}(2 \sqrt{3}-5) X_{3}^{2}-\frac{1}{4} \sqrt{3} X_{1} X_{3} \\
& \left.+\frac{1}{4} X_{2} X_{3}+\frac{1}{4} X_{1}+\frac{1}{4} \sqrt{3} X_{2}-\frac{1}{4}(2 \sqrt{3}+1) X_{3}-\frac{1}{8}\right] \\
G:= & \text { Basis }(F, \text { plex }(X[1], X[2], X[3])) \\
{[2 \sqrt{3}} & +5+(16 \sqrt{3}+14) X_{3}+13 X_{3}^{2},-\sqrt{3}+4+(26 \sqrt{3}-13) X_{3}+52 X_{2}, 8 \sqrt{3}+7 \\
& \left.+(13 \sqrt{3}-26) X_{3}+52 X_{1}\right] \\
\text { solve } & \left(2 \sqrt{3}+5+13 X_{3}^{2}+(16 \sqrt{3}+14) X_{3}, X[3]\right) \\
& -\frac{8}{13} \sqrt{3}-\frac{7}{13}-\frac{1}{13} \sqrt{176+86 \sqrt{3}},-\frac{8}{13} \sqrt{3}-\frac{7}{13}+\frac{1}{13} \sqrt{176+86 \sqrt{3}}
\end{aligned}
$$




\section{Bibliography}

[1] Bottema, O., Roth, B., Theoretical Kinematics, Dover Publications Inc., New York, NY, USA, 1990.

[2] Chen, C., "A Direct Kinematic Computation Algorithm for all Planar 3-Legged Platforms", M.Eng. Thesis, McGill University, 2001.

[3] Cox, D., Little, J., O'Shea, D., Ideals, Varieties, and Algorithms, 2nd ed., Springer-Verlag, New York, 1997.

[4] Donelan, P., S., Kinematic Singularities of Robot Manipulators, in Advances in Robot Manipulators, ed A. Lazinica, In-Tech, Vienna, Austria (2010) 401-416.

[5] Galarza, A., Seade, J., Introduction to Classical Geometries, Birkhäuser, 2000.

[6] Gosselin, C.M., Sefrioui, J., Polynomial solutions for the direct kinematic problem of planar three-degree-of-freedom parallel manipulators, IEEE Int. Conf. on Advanced Robotics, Italy, 2 (1991) 1124-1129.

[7] Hayes, M.J.D., Husty, M.L., On the kinematic constraint surfaces of general three-legged planar robot platforms, Mechanism and Machine Theory, 38 (2003) 379-394.

[8] Hayes, M.J.D., Zsombor-Murray, P.J., Chen, C., Unified kinematic analysis of general planar parallel manipulators, ASME Journal of Mechanical Design, 126(5) (2004) 866-874. 
[9] Hayes, M.J.D., "Kinematics of General Planar Stewart-Gough Platforms”, PhD. Thesis, McGill University, 1999.

[10] Husty, M., Schröcker, H-P., Algebraic Geometry and Kinematics, In Nonlinear Computational Geometry, ( eds I.Z.Emiris, F.Sottile and T.Theobald), 85-107, Springer, New York, 2010.

[11] Husty, M., Schröcker, H-P., Kinematics and Algebraic Geometry, In 21st Century Kinematics The 2012 NSF Workshop, (eds J.M.McCarthy), 85-123, Springer, London Heidelberg New York Dordrecht, 2013.

[12] Klein, F., Elementary Mathematics from an Advanced Standpoint: Geometry, Dover Publications Inc., New York, NY, USA, 1939.

[13] Merlet, J.-P., Direct kinematics of planar parallel manipulators, IEEE Int. Conf. on Robotics and Automation, Minneapolis, USA, (1996) 3744-3749.

[14] Nielsen, J., Roth, B., On the Kinematic Analysis of Robotic Mechanisms, The International Journal of Robotics Research, 18 (1999) 1147-1160.

[15] Ravani, B., Roth, B., Motion Synthesis Using Kinematic Mappings, Transactions of the ASME, 105 (1983) 460-467.

[16] Selig, J., Geometric Fundamentals of Robotics, 2nd ed., Springer, New York, 2010.

[17] Shafarevich, I., R., Basic Algebraic Geometry, Springer-Verlag, Berlin Heidelberg New York, 1977.

[18] Zsombor-Murray, P.J., Chen, C., Hayes, M.J.D., Direct Kinematic Mapping for General Parallel Manipulators, Proc. CSME Forum, Kingston, On., Canada (2002) 1-8. 